\title{
Sistemas biortogonais em espaços de Banach $C(K)$
}

\author{
Clayton Suguio Hida
}

\author{
DissertaÇÃo APRESENTADA \\ AO \\ Instituto de Matemática e Estatística \\ DA \\ Universidade de SÃo Paulo \\ PARA \\ OBTENÇÃO DO TÍTULO \\ $\mathrm{DE}$ \\ Mestre em Matemática \\ Área de Concentração: Matemática \\ Orientadora: Profa. Dra. Christina Brech
}

Durante o desenvolvimento deste trabalho o autor recebeu auxílio financeiro da FAPESP (processo 2011/15129-3).

São Paulo, Setembro de 2014 


\section{Sistemas biortogonais em espaços de Banach $C(K)$}

Esta versão da dissertação contém as correções e alterações sugeridas pela Comissão Julgadora durante a defesa da versão original do trabalho, realizada em 07/08/2014. Uma cópia da versão original está disponível no Instituto de Matemática e Estatística da Universidade de São Paulo.

Comissão Julgadora:

- Prof $^{\mathrm{a}}$. Dr ${ }^{\mathrm{a}}$. Christina Brech (orientadora) - IME-USP

- Prof. Dr. Piotr Koszmider - IMPAN

- Prof. Dr. Jorge Lopez Abad - ICMAT 


\section{Agradecimentos}

Agradeço a minha orientadora, Profa. Christina Brech, pela orientação desde a iniciação científica até a presente dissertação. Agradeço pela orientação, pelo incentivo, pela dedicação, pela paciência durante estes anos e pela imensa contribuição na minha formação acadêmica. Em especial, gostaria de agradecer pela amizade, pelo suporte e pelas oportunidades de crescimento que eu recebi durante estes anos.

Agradeço ao Prof. Piotr Koszmider pela orientação e contribuição nesta dissertação e por toda ajuda durante a minha estadia em Varsóvia.

Agradeço a todos meus amigos que direta ou indiretamente colaboraram na execução deste trabalho.

Agradeço, por fim, à FAPESP pelo apoio financeiro. 


\section{Resumo}

Hida, C. S. Sistemas biortogonais em espaços de Banach $C(K)$. 2014. xv+83f. Dissertação de mestrado - Instituto de Matemática e Estatística, Universidade de São Paulo, São Paulo, 2014.

Este trabalho tem como objetivo principal aplicar elementos de teoria dos conjuntos no estudo de sistemas biortogonais em espaços de Banach. Inicialmente, estudamos o Teorema de Markushevic, que garante que todo espaço de Banach separável admite um sistema biortogonal enumerável. Assim, partimos para o estudo de espaços de Banach não separáveis, mais especificamente, estudamos a existência de sistemas biortogonais não enumeráveis em espaços de Banach da forma $C(K)$, com $K$ compacto Hausdorff não metrizável. Nesta direção, estudamos dois teoremas devido a S. Todorcevic. O primeiro teorema nos dá condições que um compacto Hausdorff $K$ deve satisfazer de tal modo que o respectivo espaço de Banach $C(K)$ possua sistemas biortogonais não enumeráveis. O segundo teorema nos diz que, assumindo o Axioma de Martin, todo espaço de Banach não separável da forma $C(K)$ possui um sistema biortogonal não enumerável. Em seguida, consideramos algumas funções cardinais definidas por P. Koszmider para espaços de Banach, associadas aos sistemas biortogonais e estudamos suas relações com funções cardinais conhecidas. Em particular, obtemos um resultado original que relaciona o peso de um espaço compacto Hausdorff $K$ com o tamanho de tipos especiais de sistemas biortogonais em $C(K)$, generalizando um resultado de S. Todorcevic sobre álgebras de Boole. Finalmente, construímos um espaço de Ostaszewski $K$ usando o Princípio Diamante. O espaço $K$ é um compacto disperso não metrizável tal que todas suas potências finitas são hereditariamente separáveis. Este espaço é um exemplo consistente de um espaço compacto Hausdorff não metrizável tal que o respectivo espaço de Banach $C(K)$ não admite sistemas biortogonais não enumeráveis.

Palavras-chave: sistemas biortogonais, espaços de Banach $C(K)$, espaço de Ostaszewski. 


\section{Abstract}

Hida, C. S. Biorthogonal systems in Banach spaces $C(K)$. 2014. xv+83f. Dissertação de mestrado - Instituto de Matemática e Estatística, Universidade de São Paulo, São Paulo, 2014.

The main purpose of this work is to apply elements of set theory to the study of biorthogonal systems in Banach spaces. Initially, we study Markushevic's Theorem, which ensures that every separable Banach space has a countable biorthogonal system. With this result, we focus our attention to the study of nonseparable Banach spaces, more especifically, we study the existence of uncountable biorthogonal systems in Banach spaces of the form $C(K)$, with $K$ a nonmetrizable compact Hausdorff space. In this direction, we study two theorems of S. Todorcevic. The first one gives us sufficient conditions that a compact Hausdorff space $K$ must satisfy in order to get that the respective Banach space $C(K)$ has an uncountable biorthogonal system. The second one tells us that under Martin's Axiom, every nonseparable Banach space of the form $C(K)$ has an uncountable biorthogonal system. Next, we consider some cardinal functions defined by P. Koszmider for Banach spaces, related with biorthogonal systems, and we study its relations with well - known cardinal functions. In particular, we obtain an original result relating the weight of a compact Hausdorff space $K$ to the size of certain biorthogonal systems in $C(K)$, generalizing a result of S. Todorcevic for Boolean algebras. Finally, we construct an Ostaszewski space $K$ using the Diamond Principle. The compact space $K$ is a scattered nonmetrizable Hausdorff space such that all its finite powers are hereditarily separable. This space is a consistent example of a nonmetrizable compact Hausdorff space such that the respective Banach space $C(K)$ does not have an uncountable biorthogonal system.

Keywords: biorthogonal systems, Banach spaces $C(K)$, Ostaszewski space. 


\section{Sumário}

$\begin{array}{ll}\text { Lista de Abreviaturas } & \text { ix }\end{array}$

Lista de Símbolos $\quad$ xi

Introdução $\quad$ xiii

1 Preliminares $\quad 1$

1.1 Espaços de Banach . . . . . . . . . . . . . . . . . . . . . . . . 1

1.1.1 Topologias fraca e fraca estrela . . . . . . . . . . . . . . . 1

1.1.2 Espaços de Banach da forma $C(K) \ldots \ldots \ldots \ldots$

1.1 .3 Bases de Schauder . . . . . . . . . . . . . . . . . . 4

1.2 Álgebras de Boole . . . . . . . . . . . . . . . . . . . . . . 5

2 Teoria dos Conjuntos 9

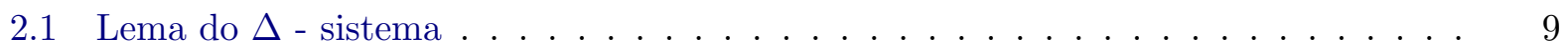

2.1.1 Uma aplicação do Lema do $\Delta$-sistema em topologia . . . . . . . . . . . . 13

2.2 Axioma de Martin . . . . . . . . . . . . . . . . . . . . . . 14

2.2 .1 Algumas aplicações do Axioma de Martin . . . . . . . . . . . . . . . . . . . . 16

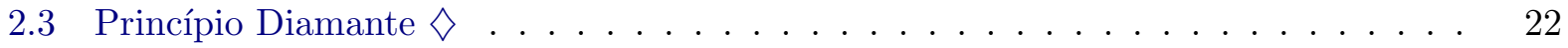

3 Sistemas biortogonais $\quad 25$

3.1 Definições e exemplos . . . . . . . . . . . . . . . . . . . 26

3.2 Existência de M-bases em espaços de Banach separáveis . . . . . . . . . . . . . . 28

3.3 Existência de sistemas biortogonais não enumeráveis em espaços $C(K)$. . . . . . 31

3.4 Não existência de sistemas biortogonais não enumeráveis em espaços $C(K)$. . . . 36

3.5 Sistemas semibiortogonais . . . . . . . . . . . . . . . . . . . . 39

4 Funções cardinais em termos de sistemas biortogonais $\quad 49$

4.1 Funções cardinais de espaços topológicos e de Banach . . . . . . . . . . . . . . . 49

4.2 Sistemas biortogonais e peso topológico . . . . . . . . . . . . . . . 51

4.3 Irredundância e biortogonalidade . . . . . . . . . . . . . . . . . 56

5 Um espaço de Ostaszewski sob o Princípio Diamante $\diamond \quad 63$

5.1 Extensões simples de álgebras enumeráveis . . . . . . . . . . . . . . . . 63

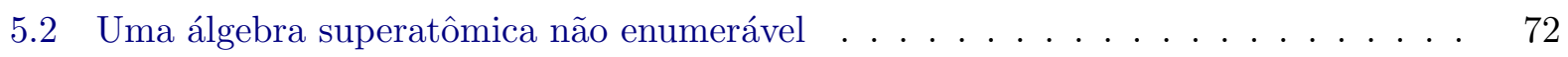

5.3 Um espaço de Ostaszewski . . . . . . . . . . . . . . . . . . . . . . . 73 
viii SUMÁRIO

5.4 Propriedades do correspondente espaço de Banach $C(K) \ldots \ldots \ldots$. . . . . 78

$\begin{array}{ll}\text { Referências Bibliográficas } & 81\end{array}$

$\begin{array}{ll}\text { Índice Remissivo } & 83\end{array}$ 


\section{Lista de Abreviaturas}

p.i.f. Propriedade da intersecção finita

c.c.c. Condição de cadeia enumerável (Countable chain condition) 


\title{
Lista de Símbolos
}

\author{
$\mathbb{N} \quad$ o conjunto dos números naturais \\ $\mathbb{R} \quad$ o conjunto dos números reais \\ $\omega$ o cardinal enumerável infinito \\ $\omega_{1} \quad$ o menor cardinal não enumerável \\ $\omega_{2} \quad$ o segundo cardinal não enumerável \\ c o cardinal do contínuo \\ $\wp(X) \quad$ o conjunto de todos os subconjuntos de $X$ \\ $|X| \quad$ a cardinalidade de $X$ \\ $f_{\mid X} \quad$ a função $f$ restrita ao conjunto $X$ \\ $\|x\| \quad$ a norma de $x$ \\ $\chi_{x} \quad$ a função característica do conjunto $x$
}




\section{Introdução}

O objetivo deste trabalho é o estudo de uma noção de sistemas de coordenadas para espaços de Banach. Em espaços vetoriais de dimensão finita, sabemos que uma base algébrica determina completamente o espaço. Como consequência do Lema de Zorn, sabemos que todo espaço vetorial de dimensão infinita também possui uma base algébrica. Porém, se temos um espaço de Banach de dimensão infinita, como consequência do Teorema de Baire, a base algébrica para esse espaço não pode ser enumerável, mesmo que o espaço de Banach seja separável. Neste sentido, considera-se uma outra noção de base, a de bases de Schauder para um espaço de Banach. Por definição, se um espaço de Banach possui uma base de Schauder, então esse espaço é necessariamente separável. Por outro lado, devido a um resultado de P.Enflo[5], existem espaços de Banach separáveis que não possuem bases de Schauder. Como pode ser observado nas linhas acima, uma importante parte dos estudos relacionados a bases está ligada à existência de bases de determinados tamanhos. Observamos ainda a importância de teoremas combinatórios, como o Lema de Zorn e o Teorema de Baire, no estudo de existência de bases.

Neste trabalho, consideramos uma outra noção de base, os chamados sistemas biortogonais. Como no caso de bases de Schauder, que podem ser considerados como uma generalização das bases algébricas, os sistemas biortogonais são uma generalização das bases de Schauder, no sentido que todo espaço que possui uma base Schauder, também possui um sistema biortogonal enumerável. O principal objetivo do trabalho é o uso de ferramentas combinatórias no estudo da existência de sistemas biortogonais. Para espaços de Banach separáveis, temos o Teorema de Markushevic, que garante a existência de sistemas biortogonais enumeráveis para espaços de Banach separáveis. Com esse resultado, o estudo se volta para espaços de Banach não separáveis. Em particular, estamos interessados no estudo de sistemas biortogonais para espaços de Banach da forma $C(K)$, com $K$ compacto não metrizável. Para esses espaços, estudamos algumas propriedades de $K$ que se refletem na existência de sistemas biortogonais em $C(K)$ ou que garantem que o espaço de Banach $C(K)$ admita apenas sistemas biortogonais de determinados tamanhos.

O presente trabalho encontra-se dividido em cinco capítulos. No Capítulo 1, é feita uma revisão de alguns aspectos essenciais de espaços de Banach e álgebras de Boole que serão utilizados no decorrer de todo o texto. Tal capítulo tem por objetivo relembrar algumas definições e introduzir notações. No Capítulo 2, estudamos algumas ferramentas combinatórias que serão utilizadas nos capítulos posteriores. Iniciamos o Capítulo 2 com o estudo do Lema do $\Delta$-sistema e uma aplicação clássica do Lema do $\Delta$-sistema em topologia. Em seguida, fazemos um estudo do Axioma de Martin, desde a definição até o estudo de algumas aplicações do Axioma de Martin em combinatória e em topologia. Encerramos o Capítulo 2 com o estudo do Princípio Diamante $\diamond$, que será usado no Capítulo 5.

No Capítulo 3, estudamos teoremas relativos à existência de sistemas biortogonais para espaços 
de Banach. Iniciamos o capítulo com a definição de sistemas biortogonais e alguns resultados básicos relativos ao comportamento dos sistemas biortogonais em relação a subespaços e aplicações lineares contínuas. Em seguida, estudamos o Teorema de Markushevic 3.1, que garante a existência de sistemas biortogonais enumeráveis para espaços de Banach separáveis com boas propriedades. Após o estudo do Teorema de Markushevic, passamos a estudar espaços de Banach não separáveis. Primeiramente, estudamos dois teoremas de S.Todorcevic que garantem a existência de sistemas biortogonais não enumeráveis em espaços de Banach da forma $C(K)$. O primeiro nos fornece propriedades suficientes que um compacto Hausdorff $K$ deve satisfazer de tal modo que o respectivo espaço de Banach $C(K)$ possua sistemas biortogonais não enumeráveis. Assim, obtemos exemplos de espaços de Banach não separáveis que admitem sistemas biortogonais não enumeráveis, partindo de uma classe de espaços compactos Hausdorff. O segundo teorema nos garante que todo espaço de Banach da forma $C(K)$ não separável possui um sistema biortogonal não enumerável, supondo o Axioma de Martin. Encerramos a seção com o enunciado do Corolário 7 do artigo [25] de S. Todorcevic que afirma que consistentemente todo espaço de Banach não separável possui sistemas biortogonais não enumeráveis.

Com os resultados da seção anterior, partimos para o estudo de exemplos consistentes de espaços de Banach não separáveis que possuem apenas sistemas biortogonais enumeráveis. Começamos estudando algumas condições que um espaço compacto Hausdorff $K$ deve satisfazer, de tal modo que o respectivo espaço de Banach $C(K)$ possua apenas sistemas biortogonais enumeráveis. Chegamos assim a alguns resultados contidos em [21]. Em particular, a existência de um espaço de Banach não separável que admita apenas sistemas biortogonais enumeráveis se relaciona com a existência de um compacto Hausdorff $K$ satisfazendo certas propriedades.

Encerramos o Capítulo 3 com uma aplicação do conceito de sistemas biortogonais ao estudo dos conjuntos suporte em espaços de Banach. Na verdade, nesta seção consideramos uma noção mais fraca de sistemas biortogonais, os denominados sistemas semibiortogonais.

No Capítulo 4, estudamos algumas funções cardinais em espaços de Banach. Destacamos nesse capítulo alguns resultados que relacionam as funções cardinais em um compacto Hausdorff $K$ com as funções cardinais associadas a sistemas biortogonais para o respectivo espaço de Banach $C(K)$. Esses resultados originais estão contidos no Teorema 4.2, que é uma generalização do Teorema 4.1 de S. Todorcevic, e no Teorema 4.6, que é uma versão do Teorema de McKenzie 4.5 para compactos Hausdorff quaisquer. O estudo das funções cardinais em espaços de Banach e suas principais relações foram baseadas no artigo de P. Koszmider [16].

Iniciamos o Capítulo 4 relembrando algumas funções cardinais definidas para espaços topológicos e introduzimos funções cardinais para espaços de Banach associadas aos sistemas biortogonais. Em seguida, estudamos, para um compacto Haussdorff $K$, as relações entre o peso de $K$ e determinados tipos de sistemas biortogonais em $C(K)$ obtendo assim o Teorema 4.2, que generaliza o Teorema 4.1 de S. Todorcevic para todo compacto Hausdorff. Concluímos o capítulo com o estudo da noção de irredundância para álgebras de Banach da forma $C(K)$. Introduzimos uma nova função cardinal associada à irredundância para espaços de Banach da forma $C(K)$ e obtemos o Teorema 4.6, que relaciona o $\pi$-peso de $K$ com a irredundância de $C(K)$.

No último capítulo, fazemos uma construção de um espaço de Ostaszewski utilizando o Princípio Diamante $\diamond$. Obtemos um espaço compacto Hausdorff disperso $K$ tal que toda potência finita de $K$ é hereditariamente separável. O espaço $K$ é um espaço de Kunen como o espaço obtido no 
Teorema 7.1 de [21]. Em particular, o espaço de Banach $C(K)$ é um exemplo consistente de espaço de Banach não separável que não possui sistemas biortogonais não enumeráveis. Além disso, todo fechado de $K$ é enumerável ou co-enumerável, sendo essa uma propriedade essencial de espaços de Ostaszewski. Como um espaço de Ostaszewski não pode ser compacto, temos que $K$ não é um espaço de Ostaszewski, porém, obtemos um subespaço $K^{*}$ de $K$ que é um espaço como o construído por A. J. Ostaszewski em [22].

Destacamos neste capítulo a importância da construção do espaço $K$. Obtemos o espaço $K$ como o espaço de Stone de uma álgebra de Boole $A$, construída utilizando-se o Princípio Diamante. Essa construção é flexível, pois as propriedades da álgebra $A$ são construídas por meio de subconjuntos densos de uma certa ordem parcial. 


\section{Capítulo 1}

\section{Preliminares}

Neste capítulo, incluímos algumas definições e resultados clássicos de espaços de Banach e de álgebras de Boole que serão utilizados no decorrer do texto.

\subsection{Espaços de Banach}

Iniciamos com um dos principais teoremas em espaços de Banach.

Teorema 1.1 (Hahn - Banach). Seja $Y$ um subespaço de um espaço normado $X$. Se $f \in Y^{*}$ então, existe $F \in X^{*}$ tal que $F_{\mid Y}=f$ e $\|F\|_{X^{*}}=\|f\|_{Y^{*}}$.

Demonstração. Ver [7], Teorema 2.2, página 55.

Do Teorema 1.1, obtemos a seguinte proposição que será utilizada no decorrer do texto:

Proposição 1.1. Seja $Y$ um subespaço fechado de um espaço normado $X$ e $x \in X \backslash Y$. Então existe um funcional $f$ de norma 1 tal que $f(x)>0$ e $f(y)=0$ para todo $y \in Y$.

Demonstração. Ver [7], Proposicão 2.7, página 57.

Teorema 1.2 (Teorema da Aplicação Aberta). Sejam $X$ e $Y$ espaços de Banach e $T: X \rightarrow Y$ linear limitado. Se T é uma aplicação sobrejetora, então $T$ é uma aplicação aberta.

Demonstração. Ver [7], Teorema 2.25, página 66.

Teorema 1.3 (Teorema do Gráfico fechado). Sejam $X, Y$ espaços de Banach e $T: X \rightarrow Y$ linear. Temos que $T$ é um operador limitado se, e somente se, o gráfico $G:=\{(x, T(x)): x \in X\}$ é fechado em $X \oplus Y$.

Demonstração. Ver [7], Teorema 2.27, página 67.

\subsubsection{Topologias fraca e fraca estrela}

Do Teorema 1.38 de [7], para um espaço de Banach $X$, temos que $B_{X}$, a bola unitária de $X$, é compacta se, e somente se, o espaço $X$ tem dimensão finita. Neste sentido, para um espaço de Banach de dimensão infinita $X$, definem - se outras topologias sobre $X$ de modo a obter propriedades interessantes, como por exemplo a compacidade de bolas fechadas limitadas. Para um espaço de Banach $X$, podemos definir a seguinte topologia:

Definição 1.1 (Topologia fraca). Seja X um espaço de Banach. Definimos a topologia fraca sobre $X$ como a topologia gerada por conjuntos da forma

$$
U\left(x_{0}, \epsilon, x_{1}^{*}, \ldots, x_{n}^{*}\right):=\left\{x \in X:\left|x_{i}^{*}\left(x_{0}\right)-x_{i}^{*}(x)\right|<\epsilon, i=1, \ldots, n\right\},
$$

onde $x_{0} \in X$, cada $x_{i}^{*} \in X^{*}$ e $\epsilon>0$.

Denotaremos por $(X, w)$ o conjunto $X$ munido da topologia fraca. 
Da definição, vemos que a topologia fraca é mais fraca que a topologia da norma, ou seja, todo aberto fraco é um aberto em norma. Apesar de que a topologia fraca possui menos abertos que a topologia da norma, não é verdade em geral que a bola unitária $B_{X}$ com a topologia fraca é compacta. Mais especificamente, $B_{X}$ com a topologia fraca é compacta se, e somente se, o espaço $X$ é reflexivo. ${ }^{1}$

Obteremos um compacto no dual de um espaço de Banach $X$, definindo uma outra topologia em $X^{*}$.

Definição 1.2 (Topologia fraca estrela). Seja X um espaço de Banach. Definimos a topologia fraca estrela sobre $X^{*}$ como a topologia gerada por conjuntos da forma

$$
U\left(x_{0}^{*}, \epsilon, x_{1}, \ldots, x_{n}\right):=\left\{x^{*} \in X:\left|x_{0}^{*}\left(x_{i}\right)-x^{*}\left(x_{i}\right)\right|<\epsilon, i=1, \ldots, n\right\},
$$

onde $x_{0}^{*} \in X^{*}$, cada $x_{i} \in X$ e $\epsilon>0$.

Denotaremos por $\left(X^{*}, w^{*}\right)$ o conjunto $X^{*}$ munido da topologia fraca estrela.

É fácil ver que todo aberto fraco estrela é um aberto fraco. Assim, a topologia fraca estrela possui menos abertos que a topologia fraca. Um dos importantes resultados sobre a topologia fraca estrela é o seguinte teorema:

Teorema 1.4 (Teorema de Alaoglu). Seja X um espaço de Banach. Então $\left(B_{\left.X^{*}, \omega^{*}\right)}\right.$ é compacto.

Demonstração. Ver [26], Teorema de Alaoglu, página 29.

Concluiremos a seção com outros resultados relativos às topologias fraca e fraca estrela que serão utilizados no decorrer do texto.

Proposição 1.2. Seja $X$ um espaço de Banach. Se $X$ é separável, então $\left(B_{X^{*}}, \omega^{*}\right)$ é metrizável.

Demonstração. Ver [26], Proposição 15, página 32.

Como consequência da Proposicao 1.2 e do Teorema de Alaoglu 1.4, segue-se que $X$ separável implica $\left(B_{X^{*}}, \omega^{*}\right)$ separável.

Lema 1.1. Sejam $\varphi_{1}, \ldots, \varphi_{n}, \varphi$ funcionais lineares em um espaço vetorial $X$. Se

$$
\bigcup_{i=1}^{n} \varphi_{i}^{-1}(0) \subset \varphi^{-1}(0)
$$

Então $\varphi$ é combinação linear de $\varphi_{1}, \ldots, \varphi_{n}$.

Demonstração. Ver [26], Lema 12, página 30.

Proposição 1.3. Seja $X$ um espaço de Banach. Então todo subspaço de dimensão finita em $X^{*} e ́$ $\omega^{*}$ - fechado.

Demonstração. Seja $Y=\operatorname{span}\left\{\varphi_{1}, \ldots, \varphi_{n}\right\}$ um subespaço de dimensão finita em $X^{*}$. Tomemos $\varphi \notin Y$. Pelo Lema 1.1, temos que existe $x \in \bigcup_{i=1}^{n} \varphi_{i}^{-1}(0) \backslash \varphi^{-1}(0)$.

Seja $\epsilon=|\varphi(x)|>0$. Assim, $\varphi \in U(\varphi, \epsilon, x)$ e $U(\varphi, \epsilon, x) \subset X \backslash Y$.

\footnotetext{
${ }^{1}$ Indicamos [26], Teorema 14, página 31.
} 


\subsubsection{Espaços de Banach da forma $C(K)$}

Nesta seção, vamos relembrar a definição de espaços de Banach da forma $C(K)$ e algumas de suas principais propriedades.

Definição 1.3. Seja $K$ um compacto Hausdorff. Seja $C(K)$ o conjunto formado por todas as funções contínuas $f: K \rightarrow \mathbb{R}$. Definamos para cada $f \in C(K)$ :

$$
\|f\|_{\infty}:=\sup _{x \in K}|f(x)|
$$

Proposição 1.4. A função $\|\cdot\|_{\infty}$ introduzida na definição anterior é uma norma e, $\left(C(K),\|\cdot\|_{\infty}\right)$ é um espaço de Banach.

Demonstração. Ver [7], Proposição 1.8, página 3.

A norma $\|\cdot\|_{\infty}$ é denominada a norma do supremo. No decorrer do texto, sempre que mencionarmos $C(K)$, estamos nos referindo ao espaço de Banach $\left(C(K),\|\cdot\|_{\infty}\right)$, a menos de menção em contrário. Em muitos casos, escreveremos apenas $\|\cdot\|$ para nos referirmos à norma $\|\cdot\|_{\infty}$.

A classe dos espaços de Banach da forma $C(K)$ é de fundamental importância no estudo dos espaços de Banach. Os espaços de Banach da forma $C(K)$ fornecem muitos exemplos clássicos, como os espaços $C([0,1]), l_{\infty}$ e $l_{\infty} / c_{0}$.

Por outro lado, uma importante consequência do Teorema de Alaoglu 1.4, é o fato de que todo espaço de Banach $X$ é isométrico a um subespaço fechado de um espaço de Banach da forma $C(K)$. De fato, basta tomarmos $K=B_{X^{*}}$ com a topologia fraca estrela e definirmos a isometria $T: X \rightarrow C(K)$ tal que $T(x)(y)=y(x)$.

Apresentaremos agora importantes resultados no contexto de espaços compactos Hausdorff $K$.

Teorema 1.5 (Lema de Urysohn). Para todo par de fechados disjuntos A, B de um espaço compacto Hausdorff $K$, existe uma função contínua $f: K \rightarrow[0,1]$ tal que, $f(x)=0$ para todo $x \in A e$ $f(x)=1$ para todo $x \in B$.

Demonstração. Ver [6], Teorema 1.5.11, página 41.

Uma importante consequência do Lema de Urysohn que usaremos frequentemente no texto é o seguinte corolário:

Corolário 1.1. Dados $K$ um compacto Hausdorff e $x, y \in K$ distintos, existe uma função $f \in$ $C(K)$ tal que $f(x)=0$ e $f(y)=1$.

Demonstração. Como $K$ é compacto Hausdorff, $\{x\}$ e $\{y\}$ são fechados em $K$. Para concluirmos, basta aplicarmos o Lema de Urysohn $1.5 \operatorname{com} A:=\{x\}$ e $B:=\{y\}$.

No caso de espaços de Banach da forma $C(K)$, faremos um frequente uso dos funcionais $\delta_{x}$.

Proposição 1.5. Seja $K$ um compacto Hausdorff. Dado $x \in K$, a aplicação $\delta_{x}: C(K) \rightarrow \mathbb{R}$ definida por $\delta_{x}(f)=f(x)$ para todo $f \in C(K)$ é um elemento de $C(K)^{*}$.

Demonstração. É fácil ver que $\delta_{x}$ é linear. Vamos verificar que $\delta_{x}$ é contínua. Dado $f \in C(K)$ temos que

$$
\left|\delta_{x}(f)\right|=|f(x)| \leq \sup _{y \in K}|f(y)|=\|f\| .
$$

Logo, $\delta_{x}$ é limitada e $\left\|\delta_{x}\right\| \leq 1$. Mais ainda, como consequência do Lema de Urysohn 1.5, existe $f_{x} \in C(K)$, tal que $\left\|f_{x}\right\|=1$ e $f_{x}(x)=1$. Assim $\left|\delta_{x}(f)\right|=1$ e, portanto, $\left\|\delta_{x}\right\| \geq 1$. Concluímos portanto que $\left\|\delta_{x}\right\|=1$.

Teorema 1.6. Seja $K$ um compacto Hausdorff. Então, o peso de $K$ é igual a densidade de $C(K)$.

Demonstração. Ver [23], Proposição 7.6.5, página 126. 
Como consequência do teorema acima, um espaço de Banach da forma $C(K)$ é separável se, e somente se, $K$ é metrizável.

Concluiremos a seção com o Teorema de Stone - Weierstrass. Precisamos antes de algumas definições.

Definição 1.4. Uma família $P \subset C(K)$ é um anel em $C(K)$ se, para todos $f, g \in P$, temos que $f+g, f-g, f \dot{g}$ também pertencem a $P$.

Definição 1.5. Uma família $P \subset C(K)$ separa pontos de $K$ se, para todos $x, y \in K$ distintos, existe $f \in P$ tal que $f(x) \neq f(y)$.

Teorema 1.7 (Stone - Weierstrass). Seja K um compacto Hausdorff. Se $P$ é um anel em $C(K)$ que contém as funções constantes e $P$ separa pontos de $K$, então $P$ é denso em $C(K)$.

Demonstração. Ver [6], Teorema 3.2.21, página 144.

\subsubsection{Bases de Schauder}

Nesta seção, relembramos a definição de base de Schauder e alguns resultados clássicos.

Definição 1.6. Seja $X$ um espaço de Banach. Um sequência $\left(e_{n}\right)_{n}$ em $X$ é uma base de Schauder se, para cada $x \in X$, existe uma única sequência de escalares $\left(\lambda_{n}\right)_{n}$ tal que

$$
x=\sum_{n \in \mathbb{N}} \lambda_{n} e_{n}\left(=\lim _{n \rightarrow \infty} \sum_{k=1}^{n} \lambda_{k} e_{k}\right) .
$$

Da definição, se $X$ é um espaço de Banach que possui uma base de Schauder, então $X$ é um espaço de Banach separável. Porém, existem espaços de Banach separáveis que não possuem bases de Schauder, como foi mostrado por P. Enflo em [5].

Teorema 1.8. Seja $X$ um espaço de Banach que possui uma base de Schauder. Então X é isomorfo a um espaço de Banach de sequências, cujos vetores unitários formam uma base de Schauder monótona.

Demonstração. Faremos aqui apenas um esboço da demonstração. Para uma demonstração completa indicamos [4], páginas 32 e 33.

Seja $X$ um espaço de Banach com base de Schauder $\left(e_{n}\right)_{n \in \omega}$. Consideremos $F$ o conjunto formado por todas as sequências $\left(\alpha_{n}\right)_{n}$ de escalares tais que $\sum_{i=0}^{\infty} \alpha_{i} e_{i}$ converge em $X$. Definamos para toda sequência de escalares $\left(\alpha_{n}\right)_{n}$ :

$$
\left\|\left|\left(\alpha_{n}\right)_{n}\left\|\mid=\sup _{m}\right\| \sum_{j=0}^{m} \alpha_{j} e_{j} \| .\right.\right.
$$

Temos que $\||\cdot|\|$ é uma norma em $F$ e $(F,\||\cdot|\|)$ é completo. Além disso, $T: F \rightarrow X$, dado por $T\left(\left(\alpha_{n}\right)_{n}\right)=\sum_{i=0}^{\infty} \alpha_{i} e_{i}$ é um isomorfismo.

Definição 1.7. Sejam $X$ um espaço de Banach e $\left(e_{n}\right)_{n}$ uma base de Schauder para X. Definimos para cada $n \in \mathbb{N}$ a projeção canônica $P_{n}: X \rightarrow X$ por

$$
P\left(\sum_{i=0}^{\infty} \lambda_{i} e_{i}\right):=\sum_{i=0}^{n} \lambda_{i} e_{i} .
$$

Teorema 1.9. Sejam $X$ um espaço de Banach e $\left(e_{n}\right)_{n}$ uma base de Schauder em $X$. As projeções canônicas $\left(P_{n}\right)_{n}$ associadas a base $\left(e_{n}\right)_{n}$ são uniformemente limitadas e a constante

$$
c:=\sup \left\{\left\|P_{n}\right\|: n \in \omega\right\}
$$

é denominada a constante da base $\left(e_{n}\right)_{n}$. 
Demonstração. Ver [7], Teorema 4.10, página 184.

Consideremos $X$ um espaço de Banach e $\left(e_{n}\right)_{n}$ uma base de Schauder para $X$. Sejam $\left(P_{n}\right)_{n}$ as projeções canônicas associadas à base $\left(e_{n}\right)_{n}$ e seja $c$ a constante da base. Para cada $n \in \omega$, definamos uma aplicação linear $f_{n}: X \rightarrow \mathbb{R}$ por

$$
f_{n}\left(\sum_{i=0}^{\infty} \lambda_{i} e_{i}\right):=\lambda_{n} .
$$

Então, para todo $x=\sum_{i=0}^{\infty} \lambda_{i} e_{i} \in X$, temos que

$$
\left\|P_{n}(x)-P_{n-1}(x)\right\|=\left\|\lambda_{n} e_{n}\right\|=\left\|f_{n}(x) e_{n}\right\|=\left|f_{n}(x)\right| \cdot\left\|e_{n}\right\|,
$$

assim,

$$
\left\|f_{n}\right\|=\sup _{x \in B_{X}}\left|f_{n}(x)\right|=\sup _{x \in B_{X}}\left\|e_{n}\right\|^{-1}\left\|P_{n}(x)-P_{n-1}(x)\right\| \leq 2 c\left\|e_{n}\right\|^{-1} .
$$

Concluímos portanto que $f_{n} \in X^{*}$ para cada $n \in \omega$. Os funcionais $f_{n}$ são denominados funcionais coordenados. Além disso, a Desigualdade 1.1.1 nos mostra que para todo $n \in \omega$

$$
\left\|e_{n}\right\| \cdot\left\|f_{n}\right\| \leq 2 c .
$$

Observemos ainda que a sequência $\left(e_{n}, f_{n}\right)_{n}$ em $X \times X^{*}$ satisfaz, para todos $i, j \in \omega$,

$$
f_{i}\left(e_{j}\right)=\delta_{i j},
$$

onde $\delta_{i j}$ é o delta de Dirac, definido por

$$
\delta_{i j}= \begin{cases}1, & \text { se } i=j \\ 0, & \text { se } i \neq j\end{cases}
$$

Nos próximos capítulos, estudaremos os sistemas biortogonais. Um sistema biortogonal para um espaço de Banach $X$ será uma sequência $\left(x_{\alpha}, x_{\alpha}^{*}\right)_{\alpha \in \Gamma}$ em $X \times X^{*}$ satisfazendo para todos $\alpha, \beta \in \Gamma$

$$
x_{\alpha}^{*}\left(x_{\beta}\right)=\delta_{\alpha \beta} .
$$

Como consequência da observação acima, seguirá que todo espaço de Banach que possui base de Schauder, em particular possui um sistema biortogonal (enumerável).

Encerramos a seção com um resultado importante para espaços de Banach separáveis. Este teorema garante a existência de bases de Schauder em algum quociente do espaço.

Teorema 1.10. Todo espaço de Banach separável de dimensão infinita possui um quociente de dimensão infinita com uma base de Schauder.

Demonstração. Ver [12], Teorema 1.9, página 303.

\section{2 Álgebras de Boole}

Nesta seção, incluímos alguns resultados sobre álgebras de Boole que serão usados nos próximos capítulos. Como mencionado na Seção 1.1.2, muitas construções de exemplos em espaços de Banach são espaços de Banach da forma $C(K)$, ou seja, estão relacionadas à construção de espaços compactos $K$. Por sua vez, as álgebras de Boole são de grande importância para construções de espaços compactos. No Capítulo 5, construiremos uma álgebra de Boole satisfazendo determinadas propriedades e, em particular, precisaremos de alguns dos resultados desta seção.

Notação 1.1. Seja $(A,+, \cdot,-, 0,1)$ uma álgebra de Boole. 
Denotaremos por $S(A)$ o espaço de Stone da álgebra A, ou seja, o conjunto formado pelos ultrafiltros de $A$ munido da topologia definida pela base de abertos $\{s(a): a \in A\}$, onde $s(a):=\{\mathfrak{u}:$ $\mathfrak{u}$ é ultrafiltro de $A$ e $a \in \mathfrak{u}\}$.

No caso de álgebras de Boole, temos o seguinte lema que tem uma função similar ao Lema de Urysohn para espaços compactos.

Lema 1.2. Seja $A$ uma álgebra de Boole e $B \subset A$ uma subálgebra própria de $A$. Dado a $\in$ $A \backslash B$ existem ultrafiltros distintos $p, q$ tais que para todo $b \in B$ temos que $\left(\chi_{s(b)}(p)=\chi_{s(b)}(q)\right)$ e $\chi_{s(a)}(p) \neq \chi_{s(a)}(q)$.

Demonstração. Seja $a \in A \backslash B$. Definimos os seguintes filtros de $B$ :

$$
F=\{b \in B: a \leq b\} \text { e } F^{\prime}=\{b \in B:-a \leq b\}
$$

Afirmação 1. $F \cup F^{\prime}$ tem p.i.f em $B$.

Demonstração. Caso contrário, existiriam $b \in F$ e $b^{\prime} \in F^{\prime}$ tais que $b \wedge b^{\prime}=0$. Como $b \in F$, segue-se que $a \leq b$ e pelo fato de que $b^{\prime} \in F^{\prime}$, segue-se que $-b \leq a$. Logo, $-b^{\prime} \leq a \leq b$.

Por outro lado, como $b \wedge b^{\prime}=0$, segue-se que $b \leq-b^{\prime}$. Assim, $b \leq a \leq b$ e portanto $a=b \in B$, uma contradição, pois $a \notin B$.

Seja $r$ um ultrafiltro de $B$ que contém $F \cup F^{\prime}$.

Afirmação 2. $r \cup\{a\}$ e $r \cup\{-a\}$ tem p.i.f em $A$.

Demonstração. De fato, suponhamos que $r \cup\{a\}$ não tem p.i.f. Então existe $c \in r$ tal que $c \wedge a=0$. Assim $a \leq-c$ e portanto $-c \in F \subset r$, uma contradição, pois $c \in r$ e $r$ tem p.i.f.

Sejam $p$ e $q$ dois ultrafiltros de $A$ contendo $r \cup\{a\}$ e $r \cup\{-a\}$ respectivamente. Então $p \neq q$ e $p \cap B=r=q \cap B$. Além disso, $\chi_{[a]}(p)=1 \neq 0=\chi_{[a]}(q)$.

Definição 1.8. Seja $A$ uma álgebra de Boole e $B \subset A$ um subconjunto. $A$ menor subálgebra de $A$ contendo $B$ será denotada por $\langle B\rangle$. Dizemos que $\langle B\rangle$ é a subálgebra de $A$ gerada por $B$.

A álgebra gerada por um conjunto $B$ é importante pois podemos determinar a forma de seus elementos em termo dos elementos de $B$. Essa forma é consequência do Teorema da Forma Normal. Antes de enunciarmos, precisamos de algumas definições.

Definição 1.9. Seja A uma álgebra de Boole.

- Dados a $\in$ A e $\epsilon$ igual a -1 ou +1 definamos $\epsilon a$ por:

$$
\epsilon a=\left\{\begin{aligned}
a, & \text { se } \epsilon=1 \\
-a, & \text { se } \epsilon=-1
\end{aligned}\right.
$$

- Dado $B \subset A$, dizemos que um elemento de $A$ é um produto elementar sobre $B$ se é um produto finito de elementos da forma $\epsilon a$, para $\epsilon \in\{1,-1\}$ e $a \in B$.

- Um elemento de A está na sua forma normal sobre $B$ se é a soma finita de elementos dois a dois disjuntos e que são produtos elementares sobre $B$.

Estamos agora em condições de enunciar o Teorema da Forma Normal:

Teorema 1.11 (Teorema da Forma Normal). Sejam A uma álgebra de Boole e B $\subset$ A. A subálgebra $\langle B\rangle$ contém exatamente os elementos de $A$ que estão na sua forma normal sobre $B$.

Demonstração. Ver [14], Proposição 4.4, página 51. 
Exemplo 1.1. Consideremos a álgebra de Boole $\wp(\mathbb{N})$, onde as operações de soma e produto são as operações de união e intersecção respectivamente. Seja $A \subset \wp(\mathbb{N})$ uma subálgebra. Dado $x \in \wp(\mathbb{N})$, pelo Teorema 1.11, segue-se que

$$
\langle A \cup\{x\}\rangle=\left\{\left(a_{1} \cap x\right) \cup\left(a_{2} \cap(\mathbb{N} \backslash x)\right): a_{1}, a_{2} \in A\right\} .
$$

Uma subálgebras da forma $\langle A \cup\{x\}\rangle$ é denominada uma extensão simples de $A$.

No Capítulo 5, estaremos interessados em uma determinada classe de álgebras de Boole e de espaços topológicos.

Definição 1.10. Uma álgebra de Boole A é superatômica se toda imagem homomórfa de A é atômica.

Definição 1.11. Um espaço topológico $X$ é disperso se todo subespaço não vazio de $X$ possui um ponto isolado.

Pela dualidade de Stone, temos a seguinte proposição:

Proposição 1.6. Uma álgebra de Boole A é superatômica se, e somente se, $S(A)$ é disperso.

Demonstração. Indicamos o artigo [3] e a Proposição 17.5 de [14].

Para as álgebras de Boole superatômicas, ainda temos o seguinte resultado:

Proposição 1.7. Se A é uma álgebra de Boole superatômica, então vale que $|A|=|S(A)|$.

Demonstração. Ver [14], Proposição 17.10, página 276. 


\section{Capítulo 2}

\section{Teoria dos Conjuntos}

Neste capítulo, estudamos resultados relacionados a teoria dos conjuntos que serão utilizados no decorrer no texto. Na Seção 2.1, estudamos a noção de $\Delta$-sistema e o importante Lema do $\Delta$-sistema 2.2. Primeiramente, faremos uma demonstração de um caso particular, e mais frequentemente usado. Em seguida, passaremos à demonstração do caso geral. Encerramos a seção com uma aplicação do Lema do $\Delta$-sistema no estudo do produto de espaços c.c.c. Durante todo o texto, o Lema do $\Delta$-sistema será muito utilizado para mostrar que determinada família tem a propriedade c.c.c.

Na Seção 2.2, estudamos o Axioma de Martin e algumas de suas aplicações clássicas. Em termos gerais, o Axioma de Martin nos garante a existência de um filtro em uma ordem parcial, e é a partir desse filtro que construímos objetos interessantes.

Na Seção 2.3, estudamos o Princípio Diamante, que será utilizado no Capítulo 5 na construção de uma álgebra de Boole. O Princípio Diamante fornece a existência de muitos conjuntos estacionários e, usando estes como conjuntos de índices, podemos fazer construções indutivas mais complexas. Iniciamos a seção relembrando as noções de conjuntos estacionários, clubs e o importante Teorema de Fodor 2.11. Em seguida, introduzimos o Princípio Diamante $\diamond$ e estudamos uma consequência dele.

\subsection{Lema do $\Delta$ - sistema}

Nesta seção, estudaremos a noção de $\Delta$-sistemas e o importante Lema do $\Delta$-sistema que será utilizado nos capítulos posteriores para determinar que dada ordem parcial satisfaz a propriedade c.c.c.

Primeiramente, demonstraremos uma versão do Lema do $\Delta$-sistema para famílias não enumeráveis de conjuntos finitos. Essa versão é mais frequentemente usada e será uma consequência da versão geral. Por fim, utilizaremos o Lema do $\Delta$-sistema para estudar o comportamento de uma propriedade topológica em relação a produtos topológicos.

Iniciamos com a seguinte definição:

Definição 2.1. Uma família $A$ de conjuntos é um $\Delta$ - sistema se, e somente se, existe um conjunto fixo $r$ chamado de raiz do $\Delta$ - sistema tal que $a \cap b=r$ para todo $a, b \in A$, com a e $b$ distintos.

Por exemplo, toda família de conjuntos dois a dois disjuntos é um $\Delta$-sistema no qual a raiz é o conjunto vazio.

Estudaremos a demonstração do Lema do $\Delta$-sistema em sua forma geral, mas antes disso, vamos estudar uma versão particular e mais frequentemente usada do Lema do $\Delta$-sistema. Para isso, precisamos do seguinte lema:

Lema 2.1. Seja A uma família não enumerável de conjuntos enumeráveis. Suponhamos que, para cada $B \subset A$ não enumerável, temos que $\bigcap B=\emptyset$. Então existe uma família não enumerável de elementos de $A$ dois a dois disjuntos. 
Demonstração. Seja $\kappa=|A|$.

Seja $A_{0} \in A$ um elemento qualquer.

Por indução, suponhamos que temos uma sequência $\left(A_{\sigma}\right)_{\sigma<\gamma}$ de elementos de $A$ satisfazendo

$$
\forall \alpha, \beta<\gamma \quad\left(\alpha \neq \beta \Rightarrow A_{\alpha} \cap A_{\beta}=\emptyset\right) .
$$

Afirmação. Existe $C \in A$ tal que $C \cap \bigcup_{\sigma<\gamma} A_{\sigma}=\emptyset$.

Demonstração da Afirmação. Suponhamos por contradição que para cada $C \in A$, temos que $C \cap$ $\bigcup_{\sigma<\gamma} A_{\sigma} \neq \emptyset$. Para cada $x \in \bigcup_{\sigma<\gamma} A_{\sigma}$, definamos $B_{x}:=\{a: a \in A$ e $x \in a\}$. Observemos que $x \in \bigcap B_{x}, \operatorname{logo} \bigcap B_{x} \neq \emptyset$. Por hipótese, todo subconjunto não enumerável de $A$ tem intersecção vazia, $\operatorname{logo}$ para todo $x \in \bigcup_{\sigma<\gamma} A_{\sigma}$ devemos ter $\left|B_{x}\right| \leq \omega$. Como para cada $C \in A$ temos que $\left(C \cap \bigcup_{\sigma<\gamma} A_{\sigma} \neq \emptyset\right)$, segue-se que $A=\bigcup\left\{B_{x}: x \in \bigcup_{\sigma<\gamma} A_{\sigma}\right\}$. Logo, $|A| \leq \omega \cdot(\omega \cdot \gamma)<\kappa$, o que é uma contradição, pois $\kappa=|A|$.

Assim, existe $C \in A$ tal que $C \cap \bigcup_{\sigma<\gamma} A_{\sigma}=\emptyset$.

Definamos então $A_{\gamma}=C$. Por recursão, $\left\{A_{\sigma}: \sigma<\kappa\right\}$ é a família procurada.

Vamos agora estudar o Lema do $\Delta$-sistema para conjuntos finitos.

Teorema 2.1 (Lema do $\Delta$-sistema). Seja A uma família não enumerável de conjuntos finitos. Então existe um subconjunto $B \subset A$ não enumerável que forma um $\Delta$-sistema.

Demonstração. Primeiramente, suponhamos que exista $n \in \omega$ tal que para cada $x \in A$ temos $(|x|=n)$.

Demonstraremos o teorema por indução em $n$. Suponha o resultado válido para todo natural menor que $n$.

- Se para todo $B \subset A$ com $|B|>\omega$ temos que $\bigcap B=\emptyset$, então segue-se do Lema 2.1 que existe $B \subset A$ com $|B|>\omega$ tal que para todos $x, y \in B$ temos $(x \neq y \Rightarrow x \cap y=\emptyset)$. Assim $B$ é um $\Delta$-sistema com raiz $r=\emptyset$.

- Suponhamos agora que existe $B \subset A$ com $|B|>\omega$ tal que $\bigcap B \neq \emptyset$. Seja $c \in \bigcap B$. Definamos $A_{1}=\{x \backslash\{c\}: x \in B\}$. Então $\left|A_{1}\right|=|B|>\omega$ e para cada $x \in A_{1}$ temos $(|x|=n-1)$. Por hipótese de indução, existe $B_{1} \subset A_{1} \operatorname{com}\left|B_{1}\right|>\omega$ e $B_{1}$ um $\Delta$-sistema de raiz $r_{1}$. Assim $B_{2}=\left\{x \cup\{c\}: x \in B_{1}\right\} \subset A$ é um $\Delta$-sistema com $\left|B_{2}\right|>\omega$ e de raiz $r=r_{1} \cup\{c\}$.

Demonstramos assim o teorema para o caso em que todos os elementos de $A$ possuem a mesma cardinalidade (finita). No caso geral, aplicamos o caso particular juntamente com a seguinte afirmação:

Afirmação. Existe $A^{\prime} \subset A$ não enumerável e $n \in \omega$ tal que, para cada $x \in A_{1}$, temos que $|x|=n$.

Demonstração da Afirmação. Para cada $n \in \omega$ definamos $A_{n}:=\{x \in A:|x|=n\}$. Então $A=$ $\bigcup_{n \in \omega} A_{n}$. Como $A$ é não enumerável, deve existir $n_{0} \in \omega$ tal que $A_{n_{0}}$ é não enumerável. Assim basta tomarmos $A^{\prime}=A_{n_{0}}$.

Exemplo 2.1. Consideremos a família $A:=\{\{o, \ldots, n\}: n \in \omega\}$. Temos que $A$ é uma família enumerável de conjuntos finitos. Porém, não existe nenhum subconjunto infinito enumerável de $A$ que é um $\Delta$-sistema.

Assim, não vale o Teorema 2.1 para familias enumeráveis de conjuntos finitos. Como veremos no Teorema 2.2, são necessárias algumas relações sobre os cardinais da família e dos conjuntos dessa família para que o resultado seja verdadeiro.

Em todo o texto, utilizaremos a versão do lema do $\Delta$-sistema contida no Teorema 2.1. Porém, essa versão é um caso particular da versão geral que passaremos a estudar. 
Teorema 2.2. Seja $\kappa$ um cardinal infinito $e \theta>\kappa$ um cardinal regular satisfazendo:

$$
\forall \alpha<\theta \quad\left(\left|\alpha^{<\kappa}\right|<\theta\right) .
$$

Suponhamos que $|A| \geq \theta$ e para cada $x \in A$ temos $(|x|<\kappa)$. Então existe $B \subset A$ tal que $|B|=\theta$ e $B$ é um $\Delta$-sistema.

Demonstração. Primeiro faremos algumas simplificações.

Afirmação 1. É suficiente supor $|A|=\theta$.

Demonstração da Afirmação 1. No caso geral, existe $f:|A| \rightarrow A$ bijeção, e basta aplicar o resultado a $f[\theta] \subset A$.

Pela Afirmação 1, vamos assumir que $|A|=\theta$.

Afirmação 2. É suficiente supor $A \subset \wp(\theta)$.

Demonstração da Afirmação 2. Como para cada $x \in A$ temos que $(|x|<\kappa)$ e $\kappa<\theta$, segue-se que $|\bigcup A| \leq \theta$. Logo, existe função injetora $f: \bigcup A \rightarrow \theta$. Seja $A_{1}=\{f[x]: x \in A\}$. Então $\left|A_{1}\right|=|A|=\theta, A_{1} \subset \wp(\theta)$ e para cada $x \in A$ temos que $(|f[x]|=|x|<\kappa)$.

Supondo o resultado válido para $A_{1}$, então existe $B_{1} \subset A_{1}$ com $\left|B_{1}\right|=\theta$ e tal que $B_{1}$ é um $\Delta$-sistema. Assim, basta tomarmos $B=\left\{f^{-1}[y]: y \in B_{1}\right\}$.

Pela Afirmação 2, vamos assumir que $A \subset \wp(\theta)$.

Lembremos que, para um conjunto bem ordenado $P$, o tipo de ordem t.o $(P)$ de $P$ é o único cardinal $\alpha$ tal que exista um isomorfismo de ordem de $\alpha$ sobre $P$.

Afirmação 3. Todo elemento $x \in A$, com a ordem herdada de $\theta$ tem tipo de ordem t.o $(x)$ menor que $\kappa$.

Demonstração da Afirmação 3. Segue-se do fato que todo isomorfismo de ordem é em particular uma função bijetora.

Pela Afirmação 3, todo $x \in A$ tem tipo de ordem menor que $\kappa$. Logo $A=\bigcup_{\xi<\kappa}\{x \in A:$ t.o $(x)=$ $\xi\}$ e esta união é disjunta. Definamos $\kappa_{\xi}:=|\{x \in A: t . o(x)=\xi\}|$. Assim, $\theta=|A|=\sum_{\xi<\kappa} \mid\{x \in$ $A: t . o(x)=\xi\} \mid=\kappa \cdot \sup \left\{\kappa_{\xi}: \xi<\kappa\right\}$. Como $\kappa<\theta$, temos que $\theta=\sup \left\{\kappa_{\xi}: \xi<\kappa\right\}$. Do fato de $\theta$ ser regular e para cada $\xi<\kappa$ temos que $\left(\kappa_{\xi} \leq \theta\right)$, deve existir $\rho<\kappa$ tal que $A_{1}=\{x \in A:$ t.o $(x)=\rho\}$ tenha cardinalidade $\theta$. Fixemos um tal $\rho$ e vamos trabalhar apenas com o subconjunto $A_{1}$.

Afirmação 4. Para todo $\alpha<\theta$, o conjunto $\left\{x: x \subset \alpha\right.$ e $\left.x \in A_{1}\right\}$ tem cardinalidade menor que $\theta$.

Demonstração da Afirmação 4. Seja $\alpha<\theta$. Suponhamos que $x \subset \alpha$ e $x \in A_{1}$. Então t.o $(x)=\rho$. Assim, existe um único isomorfismo $f: \rho \rightarrow x \subset \alpha$. Logo para cada $x \subset \alpha$ e $x \in A_{1}$, podemos associar uma única função em $\alpha^{<\kappa}$. Como $\left|\alpha^{<\kappa}\right|<\theta$, segue-se que $\left\{x: x \subset \alpha\right.$ e $\left.x \in A_{1}\right\}$ tem cardinalidade menor que $\theta$.

Afirmação 5. Temos que $\bigcup A_{1}$ é ilimitado em $\theta$.

Demonstração da Afirmação 5. De fato, se existisse $\alpha<\theta$ tal que $x<\alpha$ para todo $x \in \bigcup A_{1}$, então $\bigcup A_{1} \subset \alpha$ e, portanto, para todo $x \in A_{1}$, teríamos $x \subset \alpha$. Logo, $\left\{x: x \subset \alpha\right.$ e $\left.x \in A_{1}\right\}=A_{1}$. Mas $\left|A_{1}\right|=\theta$, contradição com a Afirmação 4 .

Para cada $x \in A_{1}$ e $\xi<\rho$, seja $x(\xi)$ o $\xi$-ésimo elemento de $x$.

Afirmação 6. Existe $\xi<\rho$ tal que $\left\{x(\xi): x \in A_{1}\right\}$ seja ilimitado em $\theta$.

Demonstração da Afirmação 6. Caso contrário, se para cada $\xi<\rho$ temos que $\left(\bigcup\left\{x(\xi): x \in A_{1}\right\}<\right.$ $\theta$ ), definamos $f: \rho \rightarrow \theta$ por $f(\xi)=\bigcup\left\{x(\xi): x \in A_{1}\right\}$. Então $\bigcup_{\xi<\rho} f(\xi)=\bigcup_{\xi<\rho} \bigcup\left\{x(\xi): x \in A_{1}\right\}=$ $\bigcup\left(\bigcup A_{1}\right)=\theta$ pela Afirmação 5. Assim $f: \rho \rightarrow \theta$ é uma aplicação cofinal, uma contradição com o fato de ser $\theta$ um cardinal regular. 
Seja então $\xi_{0}$ o menor $\xi$ tal que $\left\{x(\xi): x \in A_{1}\right\}$ é ilimitado em $\theta$. Definamos

$$
C:=\left\{x\left(\xi_{0}\right): x \in A_{1}\right\}
$$

e

$$
\alpha_{0}=\sup \left\{x(\eta)+1: x \in A_{1} \wedge \eta<\xi_{0}\right\} .
$$

Então $\alpha_{0}<\theta$ e

$$
\forall x \in A_{1} \forall \eta<\xi_{0} \quad\left(x(\eta)<\alpha_{0}\right) .
$$

Vamos definir recursivamente em $\mu<\theta$ uma sequência $x_{\mu} \in A_{1}$. Seja $x_{0} \in A_{1}$ tal que $x_{0}\left(\xi_{0}\right)>\alpha_{0}$. O elemento $x_{0}$ existe, pois o conjunto $C$ é ilimitado em $\theta$. Suponhamos definidos $x_{\nu}$ para $\nu<\mu$. Observemos que $\sup \left\{x_{\nu}(\eta): \nu<\mu \wedge \eta<\rho\right\}=\sup _{\nu<\mu} \sup _{\eta<\rho} x_{\nu}(\eta)$. Como $\theta$ é regular, $\sup _{\eta<\rho} x_{\nu}(\eta)<\theta$ para todo $\nu<\mu$, e novamente pela regularidade de $\theta$ segue-se que $\sup _{\nu<\mu} \sup _{\eta<\rho} x_{\nu}(\eta)<\theta$. Agora como $C$ é ilimitado em $\theta$, deve existir algum $x \in A_{1}$ tal que $x\left(\xi_{0}\right)>\sup \left\{x_{\nu}(\eta): \nu<\mu \wedge \eta<\rho\right\}$. Definamos então $x_{\mu}=x$.

Por indução, temos que

$$
A_{2}=\left\{x_{\mu}: \mu<\theta\right\}
$$

Por construção, $\left|A_{2}\right|=\theta$.

Afirmação 7. Se $x, y \in A_{2}$ e $x \neq y$, então $x \cap y \subset \alpha_{0}$.

Demonstração da Afirmação \%. De fato, suponhamos que $x=x_{\mu}$ e $y=x_{\nu}$ com $\nu<\mu$. Então, para cada $\forall \eta<\rho$ temos que $\left(x_{\mu}\left(\xi_{0}\right)>x_{\nu}(\eta)\right)$. Logo, para cada $\eta<\rho$ e para cada $\xi \geq \xi_{0}$, temos que $\left(x_{\mu}(\xi)>x_{\nu}(\eta)\right)$. Assim, $x \cap y \subset\left\{x_{\mu}(\xi): \xi<\xi_{0}\right\}$.

Agora, pela Equação 2.1.1, para cada $x \in A_{1}$ e para cada $\xi<\xi_{0}$, temos que $\left(x(\xi)<\alpha_{0}\right)$. Logo, $x \cap y \subset \alpha_{0}$.

Seja $r \subset \alpha_{0}$ tal que t.o $(r) \leq \rho$. Então existe um único isomorfismo $f: t . o(r) \rightarrow r \subset \alpha_{0}$. Assim, podemos associar a cada $r \subset \alpha_{0} \operatorname{com} t . o(r) \leq \rho$ uma função em $\alpha_{0}^{<\kappa}$. Como $\left|\alpha_{0}^{<\kappa}\right|<\theta$, sabemos que deve existir menos que $\theta$ de tais funções.

Seja $\wp_{\rho}\left(\alpha_{0}\right)=\left\{r \subset \alpha_{0}:\right.$ t.o $\left.(r) \leq \rho\right\}$. Então $\left|\wp_{\rho}\left(\alpha_{0}\right)\right|<\theta$. Agora

$$
\begin{gathered}
\theta=\left|A_{2}\right|=\left|\bigcup_{r \in \wp_{\rho}\left(\alpha_{0}\right)}\left\{x \in A_{2}: x \cap \alpha_{0}=r\right\}\right|=\sum_{r \in \wp_{\rho}\left(\alpha_{0}\right)}\left|\left\{x \in A_{2}: x \cap \alpha_{0}=r\right\}\right|= \\
=\left|\wp_{\rho}\left(\alpha_{0}\right)\right| \cdot \sup _{r \in \wp_{\rho}\left(\alpha_{0}\right)}\left|\left\{x \in A_{2}: x \cap \alpha_{0}=r\right\}\right| .
\end{gathered}
$$

Logo, $\theta=\sup _{r \in \wp_{\rho}\left(\alpha_{0}\right)}\left|\left\{x \in A_{2}: x \cap \alpha_{0}=r\right\}\right|$ e pela regularidade de $\theta$ deve existir $r \in \wp_{\rho}\left(\alpha_{0}\right)$ tal que $\left|\left\{x \in A_{2}: x \cap \alpha_{0}=r\right\}\right|=\theta$.

Seja então $B=\left\{x \in A_{2}: x \cap \alpha_{0}=r\right\}$. Temos que $B \subset A_{2} \subset A_{1} \subset A,|B|=\theta$ e se $x, y \in B$ $\operatorname{com} x \neq y$ então $x \cap y=r$ pela Afirmação 7 . Logo $B$ é um $\Delta$-sistema de raiz $r$.

Estudaremos agora uma variação do Teorema 2.1. Nessa variação, podemos ordenar os elementos do $\Delta$-sistema. Antes, precisamos de uma definição:

Definição 2.2. Dados dois subconjuntos $A$ e $B$ de um ordinal $\kappa$, dizemos que $A<B$ se, e somente se, $a<b$ para todo $a \in A$ e $b \in B$.

Teorema 2.3. Se $\left(F_{\alpha}\right)_{\alpha<\omega_{1}}$ é uma familia de subconjuntos finitos de $\omega_{1}$, então existe $\Gamma \in\left[\omega_{1}\right]^{\omega_{1}}$ tal que $\left(F_{\alpha}\right)_{\alpha<\Gamma}$ é um $\Delta$-sistema de raiz $\Delta e$

$$
\forall \alpha, \beta \in \Gamma \quad\left(\alpha<\beta \rightarrow \Delta<F_{\alpha} \backslash \Delta<F_{\beta} \backslash \Delta\right) .
$$

Demonstração. Pelo Teorema 2.1, podemos supor que $\left(F_{\alpha}\right)_{\alpha<\omega_{1}}$ é um $\Delta$-sistema de raiz $\Delta$.

Vamos construir $\Gamma$ por recursão. 
Temos que existe $\xi_{0}<\omega_{1}$ tal que $\min F_{\xi_{0}} \backslash \Delta>\max \Delta$. Caso contrário, defino $F: \omega_{1} \rightarrow \max \Delta$, $F(\xi)=\min F_{\xi} \backslash \Delta$. Temos que $F$ é injetora, $\log \left|\omega_{1}\right| \leq|\max \Delta| \leq \max \Delta<\omega_{1}$, o que é uma contradição.

Definamos então $G_{0}=F_{\xi_{0}}$.

Supondo definidos $\left(G_{\xi}\right)_{\xi<\kappa}$ satisfazendo

$$
\forall \alpha, \beta<\kappa\left(\alpha<\beta \rightarrow \Delta<G_{\alpha} \backslash \Delta<G_{\beta} \backslash \Delta\right) .
$$

Seja $\sigma=\sup _{\alpha<\kappa} \max G_{\alpha}$. Pela regularidade de $\omega_{1}$, temos que $\sigma<\omega_{1}$. Assim, existe $\xi_{\kappa}<\omega_{1}$ tal que $\min F_{\xi_{\kappa}} \backslash \Delta>\sigma$. Caso contrário, definamos $F: \omega_{1} \rightarrow \sigma, F(\xi)=\min F_{\xi} \backslash \Delta$. Temos portanto que $F$ é injetora, o que é uma contradição, pois $\min F_{\xi} \backslash \Delta$ é um conjunto finito. Definamos $G_{\kappa}=F_{\xi_{\kappa}}$.

Por recursão, $\Gamma=\left\{\xi_{\alpha}: \alpha<\omega_{1}\right\}$ satisfaz as condições do enunciado.

\subsubsection{Uma aplicação do Lema do $\Delta$-sistema em topologia}

Nesta seção, faremos uma aplicação do Teorema 2.1 em Topologia. Mais especificamente, estudaremos a noção de espaços topológicos com a propriedade c.c.c, e estudaremos com o auxílio do Teorema 2.1 um resultado relacionado à preservação da propriedade c.c.c para produtos arbitrários de espaços. Tais noções serão usadas na seção seguinte.

Definição 2.3. Um espaço topológico $(X, \tau)$ tem a condição de cadeia contável (c.c.c) se, e somente se, toda família de abertos não vazios dois a dois disjuntos é no máximo enumerável.

Como exemplo, todos os espaços topológicos separáveis tem c.c.c.

No caso de espaços compactos, sabemos pelo Teorema de Tychonoff que um produto topológico qualquer de espaços compactos é um espaço compacto, assim segue-se a seguinte questão:

Questão 2.1. Um produto arbitrário de espaços c.c.c é sempre um espaço c.c.c?

No caso de separabilidade, o produto arbitrário não é em geral preservado por produtos.

Exemplo 2.2. Se $\kappa>2^{\omega}$, então ${ }^{\kappa} 2$ não é separável, onde $2=\{0,1\}$ com a topologia discreta.

Demonstração. Seja $D=\left\{f_{n}: n \in \omega\right\} \subset{ }^{\kappa} 2$ um subconjunto enumerável.

Afirmação: $D$ não separa pontos de $\kappa$.

Demonstração. Suponhamos por absurdo que $D$ separa pontos de $\kappa$. Definamos para todo $\alpha<\kappa$

$$
s(\alpha)=\left(f_{n}(\alpha)\right)_{n \in \omega} .
$$

Se $\alpha<\beta$, por hipótese, existe $n \in \omega$ tal que $f_{n}(\alpha) \neq f_{n}(\beta)$. Logo $s(\alpha) \neq s(\beta)$. Temos portanto uma aplicação injetora $s: \kappa \rightarrow{ }^{2} \omega$. Como $\kappa>2^{\omega}$ chegamos a uma contradição.

Pela Afirmação, existem $\alpha<\beta$ tais que $f_{n}(\alpha)=f_{n}(\beta)$ para todo $n \in \omega$. Consideremos agora o aberto $B:=\prod_{\delta<\kappa} X_{\delta}$ em ${ }^{\kappa} 2$ onde

$$
X_{\delta}=\left\{\begin{array}{rll}
2, & \text { se } \delta \neq \alpha, \beta \\
\{0\}, & \text { se } \delta=\alpha \\
\{1\}, & \text { se } \delta=\beta
\end{array}\right.
$$

Temos então que $f_{n} \notin B$ para todo $n \in \omega$. Logo $D$ não pode ser denso em ${ }^{\kappa} 2$.

No caso dos espaços c.c.c, um resultado que auxilia na resposta à Questão 2.1 é o:

Teorema 2.4. Suponhamos que $\left\{X_{i}: i \in I\right\}$ é uma família de espaços topológicos tal que para todo $r \subset I$ finito, $\prod_{i \in r} X_{i}$ tem c.c.c. Então $\prod_{i \in I} X_{i}$ tem c.c.c 
Demonstração. Suponhamos que $\left(U_{\alpha}\right)_{\alpha<\omega_{1}}$ é uma família não enumerável de abertos não vazios dois a dois disjuntos de $\prod_{i \in I} X_{i}$.

Podemos supor que cada $U_{\alpha}$ é um aberto básico. Assim cada $U_{\alpha}$ depende de um conjunto finito $a_{\alpha} \subset I$ de coordenadas. Pelo Lema do $\Delta$-sistema 2.1, existe um subconjunto $A \subset \omega_{1}$ não enumerável tal que $\left\{a_{\alpha}: \alpha \in A\right\}$ forma um $\Delta$-sistema de raiz $r$. Temos que $r$ não pode ser vazio, pois se $a_{\alpha} \cap a_{\beta}=\emptyset$, então $U_{\alpha} \cap U_{\beta} \neq \emptyset$.

Seja $\prod\left(U_{\alpha}\right)$ a projeção de $U_{\alpha}$ em $\prod_{i \in r} X_{i}$. Então $\prod\left(U_{\alpha}\right), \alpha \in A$ é uma família não enumerável de abertos não vazios dois a dois disjuntos. Assim $\prod_{i \in r} X_{i}$ não tem c.c.c.

Corolário 2.1. Se o produto de dois espaços c.c.c é um espaço c.c.c, então o produto qualquer de espaços c.c.c é um espaço c.c.c.

O Teorema 2.4 nos mostra um comportamento distinto entre espaços separáveis e espaços c.c.c. No caso de espaços separáveis, o produto de dois espaços separáveis é sempre um espaço separável, mas este fato não nos garante que a separabilidade seja preservada por produtos arbitrários.

Com o Teorema 2.4, a questão referente a produtos arbitrários de espaços c.c.c se resume a questão de saber se o produto de dois espaços c.c.c é sempre um espaço c.c.c.

Usaremos o Teorema 2.4 na seção seguinte para mostrarmos que é consistente que o produto arbitrário de espaços c.c.c é um espaço c.c.c.

\subsection{Axioma de Martin}

Nesta seção, estudamos o Axioma de Martin e algumas de suas aplicações. Em termos gerais, o Axioma de Martin nos garante a existência de um filtro em uma ordem parcial, e é a partir desse filtro que construímos objetos interessantes. Para enunciarmos o Axioma de Martin, precisamos antes de algumas definições.

Definição 2.4. Uma ordem parcial é um par $(P, \leq)$ tal que $P \neq \emptyset$ e $\leq$ é uma relação em $P$ que é transitiva, reflexiva e antissimétrica.

Definição 2.5. Seja $(P, \leq)$ uma ordem parcial.

- Uma cadeia em $(P, \leq)$ é um conjunto $C \subset P$ tal que:

$$
\forall p, q \in C \quad(p \leq q \vee q \leq p) .
$$

- Dois elementos $p, q \in P$ são compatíveis se

$$
\exists r \in P \quad(r \leq p \wedge r \leq q) .
$$

- Dois elementos $p, q \in P$ são incompatíveis se não são compatíveis e nesse caso escrevemos $p \perp q$.

- Uma anticadeia em $P$ é um conjunto $A \subset P$ tal que:

$$
\forall p, q \in A(p \neq q \rightarrow p \perp q) .
$$

Definição 2.6. Uma ordem parcial $(P, \leq)$ tem a condição de cadeia contável (c.c.c) se, e somente se, toda anticadeia em $P$ é no máximo enumerável.

Exemplo 2.3. 1. Seja $P=\alpha$ um ordinal e $(P, \leq)$ onde $\leq e ́$ a ordem usual. Então todo subconjunto de $P$ é uma cadeia. Assim, toda anticadeia em $P$ tem cardinalidade menor ou igual a 1. Assim P tem c.c.c. 
2. Seja $X$ um conjunto não vazio e $P=\wp(X) \backslash\{\emptyset\}$ com a ordem dada pela inclusão, ou seja, $p \leq q \leftrightarrow p \subset q$. Então $p \perp q$ se, e somente se, $p \cap q=\emptyset$. Assim, $A \subset P$ é anticadeia se, e somente se, A é formado por conjuntos dois a dois disjuntos. Portanto $P$ tem c.c.c se, $e$ somente se, $|X| \leq \omega$

Definição 2.7. Seja $(P, \leq)$ uma ordem parcial.

- $D \subset P$ é denso em $P$ se, e somente se, $\forall p \in P \exists q \in D(q \leq p)$.

- $G \subset P$ é um filtro em $P$ se satisfaz:

1. $\forall p, q \in G \exists r \in G(r \leq p \wedge r \leq q) e$

2. $\forall p \in G \forall q \in P(p \leq q \rightarrow q \in G)$.

3. Dada uma família $\mathcal{D} \subset \wp(P)$ de subconjuntos densos em $P$, um $\mathcal{D}$-filtro em $P$ é um filtro $G$ em $P$ tal que:

$$
\forall D \in \mathcal{D} \quad(G \cap D \neq \emptyset) .
$$

Com as definições acima, podemos agora enunciar o Axioma de Martin.

Definição 2.8. - Seja $\kappa$ um cardinal. $M A(\kappa)$ é a sentença: Para toda ordem parcial $(P, \leq)$ c.c.c não vazia e para toda família $\mathcal{D} \subset \wp(P)$ de subconjuntos densos em $P$ com $|\mathcal{D}| \leq \kappa$, existe um $\mathcal{D}$-filtro.

- O Axioma de Martin é a sentença $M A: \forall \kappa<\mathfrak{c}(M A(\kappa))$.

Lema 2.2. $\quad 1$. se $\kappa, \sigma$ são cardinais e $\kappa<\sigma$ então $M A(\sigma) \rightarrow M A(\kappa)$.

2. $M A(\mathfrak{c})$ é falso.

3. (Rasiowa-Sikorski) $M A(\omega)$ é verdadeiro.

Demonstração. 1. Segue-se da definição.

2. Suponhamos por absurdo que vale $M A(\mathfrak{c})$.

Seja $P=\{p: p \subset \omega \times 2 \wedge|p|<\omega \wedge p$ é função $\}$ e definamos $p \leq q \leftrightarrow q \subset p$ (ou seja, se $p$ é uma extensão de $q$ ).

Então, dois elementos $p, q \in P$ são compatíveis se, e somente se, $p$ e $q$ coincidem em $\operatorname{dom}(p) \cap$ $\operatorname{dom}(q)$, sendo $p \cup q$ uma extensão comum de $p$ e $q$. Como $|P|=\omega$ segue-se que $P$ tem c.c.c. Para cada $n \in \omega$ definamos

$$
D_{n}=\{p \in P: n \in \operatorname{dom}(p)\} .
$$

Temos que cada $D_{n}$ é denso em $P$ pois, toda função finita pode ser estendida a uma função que contenha $n$ em seu domínio.

Para cada função $h: \omega \rightarrow 2$ definamos

$$
E_{h}=\{p \in P: \exists n \in \operatorname{dom}(p)(p(n) \neq h(n))\} .
$$

Temos que cada $E_{h}$ é denso em $P$ pois, toda função finita pode ser estendida a uma função que contenha $n$ em seu domínio e tal que o valor da extensão em $n$ seja diferente de $h(n)$.

Seja agora

$$
\mathcal{D}=\left\{D_{n}: n \in \omega\right\} \cup\left\{E_{h}: h: \omega \rightarrow 2 \text { função }\right\} .
$$

Por $M A(\mathfrak{c})$ seja $G$ um $\mathcal{D}$-filtro em $P$. Como $G$ é filtro, $G$ é um conjunto formado por funções duas a duas compatíveis, assim $f_{G}=\bigcup G$ é uma função com $\operatorname{dom}\left(f_{G}\right) \subset \omega$. Por hipótese, para cada $n \in \omega$ temos que $G \cap D_{n} \neq \emptyset$, $\operatorname{logo} \operatorname{dom}\left(f_{G}\right)=\omega$. Assim $f_{G}: \omega \rightarrow 2$. Agora, por hipótese, para cada $h \in^{\omega} 2$, temos que $G \cap E_{h} \neq \emptyset$, logo $f_{G} \neq h$ para toda $h: \omega \rightarrow 2$ e, em particular, $f_{G} \neq f_{G}$, o que é uma contradição. 
3. (Rasiowa-Sikorski)Seja $(P, \leq)$ uma ordem parcial e $\mathcal{D}=\left\{D_{n}: n \in \omega\right\}$ uma família de conjuntos densos em $P$.

Seja $p_{0}$ um elemento qualquer de $P$. Supondo definidos $p_{0} \geq p_{1} \geq \ldots \geq p_{n}$ seja $p_{n+1} \in D_{n+1}$ tal que $p_{n} \geq p_{n+1}$. A existência de $p_{n+1}$ é garantida pelo fato de ser $D_{n+1}$ denso em $P$.

Por indução, temos uma família $E=\left\{p_{n}: n \in \omega\right\}$. Seja $G$ o filtro gerado por $E$, ou seja

$$
G=\left\{q \in P: \exists n \in \omega\left(q \geq p_{n}\right)\right\}
$$

Temos então que $G$ é um $\mathcal{D}$-filtro em $P$, já que para todo $n \in \omega$ temos $p_{n} \in G \cap D_{n}$, ou seja, $G$ intersecta cada elemento da família $\mathcal{D}$.

Como consequência da parte 3) do lema anterior, se vale a Hipótese do Contínuo, ou seja, $\omega_{1}=\mathfrak{c}$, então vale $M A$. Assim, temos que

$$
C H \rightarrow M A \text {. }
$$

Como $C H$ é consistente com $Z F C$, concluímos que $M A$ é uma axioma consistente com $Z F C$. Mais ainda, pelo Corolário 6.4 de [17], temos que $M A$ é independente de $Z F C$.

Observemos ainda que na parte 3) do lema anterior, não usamos a propriedade c.c.c. Vamos mostrar agora que não podemos retirar a propriedade c.c.c para $M A(\kappa) \operatorname{com} \kappa>\omega$.

Exemplo 2.4. Consideremos

$$
P=\left\{p: p \subset \omega \times \omega_{1} \wedge|p|<\omega \wedge p \text { é função }\right\} .
$$

Com a ordem definida por

$$
p \leq q \leftrightarrow q \subset p .
$$

Definamos para cada $\alpha<\omega_{1}$ o conjunto

$$
D_{\alpha}=\{p \in P: \alpha \in \operatorname{Img}(p)\} .
$$

Temos assim que cada $D_{\alpha}$ é denso em P. Seja $\mathcal{D}=\left\{D_{\alpha}: \alpha<\omega_{1}\right\}$. Supondo-se que exista $\mathcal{D}$-filtro $G$ em $P$, então $f_{G}=\bigcup G$ é uma função sobrejetora de $\omega$ sobre $\omega_{1}$, uma contradição. Observemos que $\left\{(0, \alpha): \alpha<\omega_{1}\right\}$ é uma familia de cardinalidade $\omega_{1}$ formada por elementos dois a dois incompativeis, $\operatorname{logo} P$ não tem c.c.c.

\subsubsection{Algumas aplicações do Axioma de Martin}

Apresentaremos nesta seção algumas aplicações clássicas do Axioma de Martin.

Para as primeiras aplicações do Axioma de Martin, precisamos antes obter um resultado auxiliar. Esse resultado nos garante que, dados dois subconjuntos $A, C \subset \wp(\omega)$ satisfazendo certas condições, existe um subconjunto infinito $d \subset \omega$ que é quase disjunto de todo elemento de $A$ e que possui intersecção infinita com cada elemento de $C$. Esse é o conteúdo do Teorema 2.5 que será apresentado mais adiante.

Para a demonstração do Teorema 2.5, vamos trabalhar com a seguinte ordem parcial:

Definição 2.9. Seja $A \subset \wp(\omega)$. Definamos $\left(P_{A}, \leq\right)$ por:

$$
P_{A}=\{\langle s, F\rangle: s \subset \omega \wedge|s|<\omega \wedge F \subset A \wedge|F|<\omega\} .
$$

$e$

$$
\left\langle s^{\prime}, F^{\prime}\right\rangle \leq\langle s, F\rangle \leftrightarrow s \subset s^{\prime} \wedge F \subset F^{\prime} \wedge \forall x \in F\left(x \cap s^{\prime} \subset s\right) .
$$


Lema 2.3. Em $P_{A}$, dois elementos $\left\langle s_{1}, F_{1}\right\rangle$ e $\left\langle s_{2}, F_{2}\right\rangle$ são compativeis se, e somente se,

$$
\forall x \in F_{1} \quad\left(x \cap s_{2} \subset s_{1}\right) \wedge \forall x \in F_{2}\left(x \cap s_{1} \subset s_{2}\right)
$$

e neste caso, $\left\langle s_{1} \cup s_{2}, F_{1} \cup F_{2}\right\rangle$ é uma extensão comum.

Demonstração. Suponhamos que $\left\langle s_{1}, F_{1}\right\rangle$ e $\left\langle s_{2}, F_{2}\right\rangle$ são compatíveis e seja $\langle s, F\rangle$ uma extensão comum.

Se $x \in F_{1}$, então $x \cap s_{2} \subset x \cap s \subset s_{1}$, pois $s_{2} \subset s$ e $\forall x \in F_{1}\left(x \cap s \subset s_{1}\right)$. De modo análogo, se $x \in F_{2}$ então $x \cap s_{1} \subset s_{2}$.

Reciprocamente, suponhamos que

$$
\forall x \in F_{1} \quad\left(x \cap s_{2} \subset s_{1}\right) \wedge \forall x \in F_{2}\left(x \cap s_{1} \subset s_{2}\right)
$$

e consideremos $\left\langle s_{1} \cup s_{2}, F_{1} \cup F_{2}\right\rangle$. É fácil ver que $\left\langle s_{1} \cup s_{2}, F_{1} \cup F_{2}\right\rangle$ é um elemento de $P_{A}$. Seja $x \in F_{1}$. Então $x \cap\left(s_{1} \cup s_{2}\right)=\left(x \cap s_{1}\right) \cup\left(x \cap s_{2}\right) \subset s_{1}$ pois vale que $\forall x \in F_{1}\left(x \cap s_{2} \subset s_{1}\right)$. Logo $\left\langle s_{1} \cup s_{2}, F_{1} \cup F_{2}\right\rangle$ é uma extensão de $\left\langle s_{1}, F_{1}\right\rangle$. De modo análogo se mostra que $\left\langle s_{1} \cup s_{2}, F_{1} \cup F_{2}\right\rangle$ é também uma extensão de $\left\langle s_{2}, F_{2}\right\rangle$. Assim, $\left\langle s_{1} \cup s_{2}, F_{1} \cup F_{2}\right\rangle$ é uma extensão comum.

Definição 2.10. Seja $G$ um filtro em $P_{A}$. Definamos

$$
d_{G}:=\bigcup\{s: \exists F(\langle s, F\rangle \in G)\} .
$$

Lema 2.4. Seja $G$ um filtro em $P_{A}$. Então, se $\langle s, F\rangle \in G$ temos que

$$
\forall x \in F\left(x \cap d_{G} \subset s\right) .
$$

Demonstração. Suponhamos que $\left\langle s^{\prime}, F^{\prime}\right\rangle \in G$. Então $\langle s, F\rangle$ e $\left\langle s^{\prime}, F^{\prime}\right\rangle$ são compatíveis, e pelo Lema 2.3, $\forall x \in F\left(x \cap s^{\prime} \subset s\right)$. Logo $\forall x \in F\left(x \cap d_{G} \subset s\right)$.

Definição 2.11. Se $x \in A$, definamos $D_{x}:=\left\{\langle s, F\rangle \in P_{A}: x \in F\right\}$.

Lema 2.5. Se $G$ é um filtro em $P_{A}$ e $G \cap D_{x} \neq \emptyset$, então $\left|x \cap d_{G}\right|<\omega$.

Demonstração. Seja $\langle s, F\rangle \in G \cap D_{x}$. Então $x \in F$ e pelo Lema $2.4, x \cap d_{G} \subset s$. Logo $\left|x \cap d_{G}\right| \leq$ $|s|<\omega$.

Lema 2.6. Se $x \in A$, então $D_{x}$ é denso em $P_{A}$.

Demonstração. Seja $\langle s, F\rangle \in P_{A}$. Consideremos $\langle s, F \cup\{x\}\rangle \in D_{x}$. Temos claramente que $s \subset s$ e $F \subset F \cup\{x\}$. Além disso, para todo $y \in F$, temos que $(y \cap s \subset s)$. $\operatorname{Logo}\langle s, F \cup\{x\}\rangle \leq\langle s, F\rangle$.

Lema 2.7. $P_{A}$ tem c.c.c.

Demonstração. Seja $S=\left\{\left\langle s_{\xi}, F_{\xi}\right\rangle: \xi<\omega_{1}\right\}$ uma anticadeia. Consideremos a família $\left\{s_{\xi}: \xi<\omega_{1}\right\}$.

Se existissem $\alpha<\gamma<\omega_{1}$ tais que $s_{\alpha}=s_{\gamma}$, pelo Lema 2.3, teríamos que $\left\langle s_{\alpha}, F_{\alpha}\right\rangle$ e $\left\langle s_{\gamma}, F_{\gamma}\right\rangle$ seriam compatíveis. Assim, $\left\{s_{\xi}: \xi<\omega_{1}\right\}$ seria uma família de subconjuntos finitos de $\omega$ de cardinalidade $\omega_{1}$, uma contradição, pois o conjunto formado por todos os subconjuntos finitos de $\omega$ tem cardinalidade $\omega$.

Se $|A| \leq \kappa$ e vale $M A(\kappa)$, então existe um filtro $G$ em $P_{A}$ que intersecta $D_{x}$ para todo $x \in A$.

Assim, pelo Lema 2.5, $d_{G}$ é quase disjunto de todo elemento de $A$. Observemos porém que se existe $F \subset A$ finito tal que $\bigcup F$ é cofinito, então não pode existir um filtro $G$ em $P_{A}$ tal que $d_{G}$ seja infinito e quase disjunto de cada elemento de $F$.

Teorema 2.5. $M A(\kappa)$. Sejam $A, C \subset \wp(\omega)$ com $|A| \leq \kappa,|C| \leq \kappa$ e suponhamos que para todo $y \in C$ e para todo $F \subset A$ finito, temos que $|y \backslash \bigcup F|=\omega$. Então existe $d \subset \omega$ tal que

$$
\forall x \in A \quad(|d \cap x|<\omega) e \forall y \in C \quad(|d \cap y|=\omega)
$$


Demonstração. Para $y \in C$ e $n \in \omega$ definamos

$$
E_{n}^{y}:=\left\{\langle s, F\rangle \in P_{A}: s \cap y \not \subset n\right\} .
$$

Afirmação. Para todo $n \in \omega$ e $y \in C$, temos que $E_{n}^{y}$ é denso em $P_{A}$.

Demonstração. De fato, dado $\langle s, F\rangle \in P_{A}$, por hipótese $|y \backslash \bigcup F|=\omega$. Logo existe $m>n$ com $m \in y \backslash \bigcup F$. Seja $\langle s \cup\{m\}, F\rangle \in P_{A}$. Então $\langle s \cup\{m\}, F\rangle \in E_{n}^{y}$ e dado $x \in F$ temos que $x \cap(s \cup\{m\})=x \cap s \subset s$. Logo $\langle s \cup\{m\}, F\rangle \leq\langle s, F\rangle$.

Pelo Lema 2.7, $P_{A}$ tem c.c.c. Como estamos assumindo $M A(\kappa)$, seja $G$ um filtro em $P_{A}$ intersectando cada conjunto denso em

$$
\left\{D_{x}: x \in A\right\} \cup\left\{E_{n}^{y}: y \in C \wedge n \in \omega\right\} .
$$

Pela observação anterior ao teorema, para cada $x \in A$ temos que $\left(\left|d_{G} \cap x\right|<\omega\right)$. Agora se $y \in C$, dado $n \in \omega$, como $G \cap E_{n}^{y} \neq \emptyset$, então $d_{G} \cap y \not \subset n$. Assim $d_{G} \cap y$ não pode ser finito, logo $\left|d_{G} \cap y\right|=\omega$.

Com o auxílio do Teorema 2.5, vamos iniciar o estudo de algumas aplicações do Axioma de Martin.

Aplicação 1: Se $A \subset \wp(\omega)$ é família quase disjunta ${ }^{1}$ e $|A| \leq \kappa<\mathfrak{c}$, então A não é uma família quase disjunta maximal.

Proposição 2.1. Seja $A \subset \wp(\omega)$ uma familia quase disjunta de cardinalidade $\kappa$, com $\omega \leq \kappa<\mathfrak{c}$. Se vale $M A(\kappa)$, então A não é maximal.

Demonstração. Definamos $C=\{\omega\}$. Se $F \subset A$ é finito, então $|\omega \backslash \bigcup F|=\omega$. De fato, se tivéssemos $|\omega \backslash \bigcup F|<\omega$, então dado $a \in A \backslash F$, necessariamente $a$ seria finito, o que é uma contradição com o fato de que os elementos de $A$ tem cardinalidade $\omega$.

Assim, pelo Teorema 2.5, existe $d \in[\omega]^{\omega}$ quase disjunto de todo elemento de $A$.

Aplicação 2: $\omega \leq \kappa<\mathfrak{c} \rightarrow 2^{\kappa}=\mathfrak{c}$.

Lema 2.8. Seja $B \subset \wp(\omega)$ uma familia quase disjunta de tamanho $\kappa$, com $\omega \leq \kappa<\mathfrak{c}$. Seja $A \subset B$. Se $M A(\kappa)$, então existe $d \subset \omega$ tal que

$$
\forall x \in A \quad(|d \cap x|<\omega) e \forall x \in B \backslash A \quad(|d \cap x|=\omega) .
$$

Demonstração. Segue-se do Teorema 2.5, com $C=B \backslash A$, observando que se existe $F \subset A$ e $y \in B$ tal que $|y \backslash \bigcup F|<\omega$ então $|y|<\omega$, o que é uma contradição, pois todo elemento de $B$ possui cardinalidade $\omega$.

Teorema 2.6. $M A(\kappa) \rightarrow 2^{\kappa}=\mathfrak{c}$.

Demonstração. Seja $B \subset \wp(\omega)$ uma família quase disjunta de tamanho $\kappa$. Definamos $\Phi: \wp(\omega) \rightarrow$ $\wp(B)$ por $\Phi(d)=\{x \in B:|x \cap d|<\omega\}$.

Seja $A \in \wp(B)$. Pelo Lema 2.8, existe $d \subset \omega$ tal que

$$
\forall x \in A \quad(|x \cap d|<\omega) \text { e } \forall x \in B \backslash A \quad(|s \cap d|=\omega) .
$$

Assim, $A=\Phi(d)$. Logo $\Phi$ é sobrejetora. Portanto

$$
|\wp(B)|=2^{\kappa} \leq|\wp(\omega)|=\mathfrak{c} .
$$

\footnotetext{
${ }^{1}$ Dizemos que $A$ é uma família quase disjunta se, para todos distintos $a, b \in A$, temos que $|a \cap b|<\omega$.
} 
Corolário 2.2. $M A \rightarrow \mathfrak{c}$ é regular.

Demonstração. Se $\omega \leq \kappa<\mathfrak{c}$, então $2^{\kappa}=\mathfrak{c}$. Pelo Teorema de König ${ }^{2}$ segue-se que

$$
c f(\mathfrak{c})=c f\left(2^{\kappa}\right)>\kappa .
$$

\section{Aplicações em topologia.}

Definição 2.12. Seja $(X, \tau)$ um espaço topológico. Dizemos que $A \subset X$ é um conjunto de primeira categoria se existem $\left(F_{n}\right)_{n}$ subconjuntos fechados de interior vazio tais que $A \subset \bigcup_{n} F_{n}$.

O seguinte teorema é uma generalização do Teorema de Baire para a reta real.

Teorema 2.7. $M A(\kappa)$. Sejam $\left\{M_{\alpha}: \alpha<\kappa\right\}$ subconjuntos de primeira categoria de $\mathbb{R}$. Então $\bigcup_{\alpha<\kappa} M_{\alpha}$ é de primeira categoria.

Demonstração. Temos que cada $M_{\alpha}$ está contido em uma união enumerável de conjuntos fechados de interior vazio. Assim é suficiente mostrarmos que se $\left\{K_{\alpha}: \alpha<\kappa\right\}$ são subconjuntos fechados de interior vazio, então existem $H_{n}, n<\omega$ subconjuntos fechados de interior vazio tais que

$$
\bigcup_{\alpha<\kappa} K_{\alpha} \subset \bigcup_{n<\omega} H_{n}
$$

Agora, tomando complementos, para mostrarmos a inclusão acima, basta mostrarmos que se $\left\{U_{\alpha}: \alpha<\kappa\right\}$ é uma família de abertos densos, então existem $V_{n}, n<\omega$ abertos densos tais que

$$
\bigcap_{n \in \omega} V_{n} \subset \bigcap_{\alpha<\kappa} U_{\alpha}
$$

Seja $\left(B_{i}\right)_{i<\omega}$ uma enumeração de todos os intervalos abertos não vazios de $\mathbb{R}$ com extremidades racionais. Temos assim que $\left(B_{i}\right)_{i<\omega}$ é uma base para $\mathbb{R}$.

Definamos para todo $n \in \omega$

$$
V_{n}:=\bigcup\left\{B_{i}: i \in d \wedge i>n\right\}
$$

para uma particular escolha do subconjunto $d \subset \omega$. Essa escolha é feita por meio do Teorema 2.5.

Para todo $j<\omega$ definamos

$$
c_{j}=\left\{i \in \omega: B_{i} \subset B_{j}\right\} .
$$

Se $\left|d \cap c_{j}\right|=\omega$, então para todo $n \in \omega$, existe $i>n$ com $i \in d$ e $i \in c_{j}$, ou seja, $i \in d$ e $B_{i} \subset B_{j}$. Assim, $V_{n} \cap B_{j} \neq \emptyset$.

Portanto, se para todo $j<\omega$ temos que $\left|d \cap c_{j}\right|=\omega$ então, $\forall j, n<\omega\left(V_{n} \cap B_{j} \neq \emptyset\right)$, ou seja, cada $V_{n}$ é denso em $\mathbb{R}$.

Para cada $\alpha<\kappa$ definamos

$$
a_{\alpha}=\left\{i \in \omega: B_{i} \not \subset U_{\alpha}\right\} .
$$

Se $\left|d \cap a_{\alpha}\right|<\omega$, então existe $n \in \omega$ tal que $d \cap a_{\alpha} \subset n$. Assim, se $i>n$ e $i \in d$, então $i \notin a_{\alpha}$, ou seja, $B_{i} \subset U_{\alpha}$.

Logo, se $i>n$ e $i \in d$, então $B_{i} \subset U_{\alpha}$. Portanto, $V_{n} \subset U_{\alpha}$.

Assim, se para cada $\alpha<\kappa$ temos que $\left(\left|d \cap a_{\alpha}\right|<\omega\right)$, então

$$
\bigcap_{n<\omega} V_{n} \subset \bigcap_{\alpha<\kappa} U_{\alpha} .
$$

\footnotetext{
${ }^{2}$ Se $\lambda \geq \omega, c f\left(2^{\lambda}\right)>\lambda$.
} 
Seja então $C=\left\{c_{n}: n \in \omega\right\}$ e $A=\left\{a_{\alpha}: \alpha<\kappa\right\}$. Se $F \subset \kappa$ é finito, então

$$
c_{j} \backslash \bigcup_{\alpha \in F} a_{\alpha}=\left\{i \in \omega: B_{i} \subset\left(B_{j} \cap \bigcap_{\alpha \in F} U_{\alpha}\right)\right\}
$$

é infinito, pois $\bigcup_{\alpha \in F} U_{\alpha}$ é aberto denso e $B_{j}$ é aberto não vazio. Assim, para todo $F \subset \kappa$ finito e para todo $j<\omega$ temos que $\left|c_{j} \backslash \bigcup_{\alpha \in F} a_{\alpha}\right|=\omega$.

Pelo Teorema 2.5, existe $d \subset \omega$ tal que

$$
\forall \alpha<\kappa\left(\left|d \cap a_{\alpha}\right|<\omega\right) \text { e } \forall n<\omega\left(\left|d \cap c_{n}\right|=\omega\right) .
$$

Estudaremos agora um uma consequência de $M A(\kappa)$ referente a espaços compactos Hausdorff com a propriedade c.c.c. Na verdade, essa consequência é equivalente a $M A(\kappa)^{3}$.

Teorema 2.8. $M A(\kappa)$. Seja $(X, \tau)$ um espaço compacto Hausdorff c.c.c e $U_{\alpha}, \alpha<\kappa$ subconjuntos densos de $X$. Então $\bigcap_{\alpha<\kappa} U_{\alpha} \neq \emptyset$

Demonstração. Seja $P=\tau \backslash\{\emptyset\} \operatorname{com} p \leq q \leftrightarrow p \subset q$.

Temos então que $p \perp q \leftrightarrow p \cap q=\emptyset$. Assim, como $X$ tem c.c.c, segue-se que $P$ tem c.c.c.

Definamos para cada $\alpha<\kappa$

$$
D_{\alpha}=\left\{p \in P: \bar{p} \subset U_{\alpha}\right\} .
$$

Afirmação: Para todo $\alpha<\kappa, D_{\alpha}$ é denso em $P$.

Demonstração da Afirmação. Seja $p \in P$. Como $U_{\alpha}$ é denso, $U_{\alpha} \cap p$ é aberto não vazio. Assim, existe aberto $q \subset X$ e $x \in q$ com $q \subset U_{\alpha} \cap p$. Pela regularidade de $X$, existem abertos $A$ e $B$ com $X \backslash q \subset A, x \in B$ e $A \cap B=\emptyset$. Em particular, $B \subset X \backslash A \subset q$ e como $X \backslash A$ é fechado, $\bar{B} \subset q$. Logo, $\bar{B} \subset q \subset U_{\alpha} \cap p$. Assim, $B \in D_{\alpha}$ e $B \leq p$. Portanto, $D_{\alpha}$ é denso em $P$.

Por $M A(\kappa)$, seja $G$ um filtro em $P$ intersectando cada elemento da família $\left\{D_{\alpha}: \alpha<\kappa\right\}$.

Como $G$ é filtro, $G$ tem a propriedade da intersecção finita. Como $X$ é compacto, segue-se que $\bigcap\{\bar{p}: p \in G\} \neq \emptyset$.

Pela escolha do filtro $G$, para cada $\alpha<\kappa$, existe $p \in G \cap D_{\alpha}$. Logo,

$$
\emptyset \neq \bigcap\{\bar{p}: p \in G\} \subset \bigcap_{\alpha<\kappa} U_{\alpha} .
$$

Vamos estudar agora o produto de espaços c.c.c.

Lema 2.9. $M A\left(\omega_{1}\right)$. Seja $(X, \tau)$ um espaço c.c.c e $\left\{U_{\alpha}: \alpha<\omega_{1}\right\}$ uma família de abertos não vazios de $X$. Então existe um subconjunto não enumerável $A \subset \omega_{1}$ tal que $\left\{U_{\alpha}: \alpha \in A\right\}$ tem a propriedade da intersecção finita.

Demonstração. Definamos para $\alpha<\omega_{1}$

$$
V_{\alpha}=\bigcup_{\gamma>\alpha} U_{\gamma}
$$

Então $\alpha<\beta \rightarrow V_{\beta} \subset V_{\alpha}$.

Afirmação 1: Existe $\alpha<\omega_{1}$ tal que

$$
\forall \beta>\alpha\left(\bar{V}_{\beta}=\bar{V}_{\alpha}\right)
$$

\footnotetext{
${ }^{3}$ Indicamos [17], Teorema 3.4, página 65.
} 
Demonstração da Afirmação 1. Caso contrário, definamos uma sequência crescente de ordinais $\left(a_{\xi}\right)_{\xi<\omega_{1}}$ por indução.

- $a_{o}=0$.

- Supondo definido $a_{\eta}$, como não vale a afirmação, existe $\alpha>a_{\eta}$ tal que $\bar{V}_{\alpha} \neq \bar{V}_{a_{\eta}}$. Definamos então $a_{\eta+1}=\alpha$.

- Se $\gamma<\omega_{1}$ é limite, seja $a_{\gamma}=\sup \left\{a_{\xi}: \xi<\gamma\right\}$.

Por indução transfinita temos uma sequência $\left(a_{\xi}\right)_{\xi<\omega_{1}}$ de ordinais crescentes que satisfaz $\forall \xi<$ $\omega_{1}\left(\bar{V}_{a_{\xi+1}} \neq \bar{V}_{a_{\xi}}\right)$. Assim, $\forall \xi<\omega_{1}\left(V_{a_{\xi}} \backslash \bar{V}_{a_{\xi+1}} \neq \emptyset\right)$.

Logo, $\left\{V_{a_{\xi}} \backslash \bar{V}_{a_{\xi+1}}: \xi<\omega_{1}\right\}$ é um família de abertos não vazios dois a dois disjuntos, uma contradição, pois $X$ tem c.c.c. Logo, a Afirmação 1 é verdadeira.

Fixemos um $\alpha<\omega_{1}$ tal que

$$
\forall \beta>\alpha\left(\bar{V}_{\beta}=\bar{V}_{\alpha}\right) .
$$

Seja $P=\left\{p \subset V_{\alpha}: p\right.$ é aberto não vazio $\}$ com $p \leq q \leftrightarrow p \subset q$. Como $X$ tem c.c.c, segue-se que $P$ tem c.c.c.

Se $G$ é filtro em $P$, então $G$ tem a p.i.f. Assim, se

$$
A=\left\{\gamma<\omega_{1}: \exists p \in G\left(p \subset U_{\gamma}\right)\right\} .
$$

Então, $\left\{U_{\alpha}: \alpha \in A\right\}$ tem a p.i.f.

Vamos tomar um filtro $G$ de tal modo que $A$ como definido acima seja não enumerável.

Para $\beta<\omega_{1}$ definamos

$$
D_{\beta}=\left\{p \in P: \exists \gamma>\beta\left(p \subset U_{\gamma}\right)\right\} .
$$

Afirmação 2: Para todo $\beta<\omega_{1}, D_{\beta}$ é denso em $P$.

Demonstração da Afirmação 2. Como vale a 2.2.1, segue-se que $\bar{V}_{\alpha} \subset \bar{V}_{\beta}$. Se $p \in P$, então $p \subset$ $V_{\alpha} \subset \bar{V}_{\beta}$ e portanto $p \cap V_{\beta} \neq \emptyset$. Logo deve existir $\gamma>\beta$ tal que $p \cap U_{\gamma} \neq \emptyset$ (pela definição de $V_{\beta}$ ).

Assim, $p \cap U_{\gamma}$ é uma extensão de $p$ em $D_{\beta}$.

Agora, por $M A\left(\omega_{1}\right)$, existe $G$ filtro em $P$ tal que $\forall \beta<\omega_{1}\left(G \cap D_{\beta} \neq \emptyset\right)$. Assim, para todo $\beta<\omega_{1}$, como $G \cap D_{\beta} \neq \emptyset$, existem $p \in G$ e $\gamma>\beta$ tais que $p \subset U_{\gamma}$. Portanto, $\gamma \in A$. Logo, $A$ é ilimitado em $\omega_{1}$, e como $\omega_{1}$ é regular, $|A|=\omega_{1}$.

Teorema 2.9. $M A\left(\omega_{1}\right)$. O produto qualquer de espaços c.c.c é um espaço c.c.c.

Demonstração. Pelo Corolário 2.1, é suficiente mostrar que o produto de dois espaços c.c.c é sempre um espaço c.c.c.

Sejam $X$ e $Y$ espaços c.c.c. e suponhamos que $X \times Y$ não seja c.c.c.

Seja $\left\{W_{\alpha}: \alpha<\omega_{1}\right\}$ uma família de abertos disjuntos não vazios de $X \times Y$. Para cada $\alpha<\omega_{1}$, tomemos um produto de abertos não vazios $U_{\alpha} \times V_{\alpha} \subset W_{\alpha}$.

Pelo lema anterior, existe $A \subset \omega_{1}$ não enumerável tal que $\left\{U_{\alpha}: \alpha \in A\right\}$ tem p.i.f. Assim, se $\alpha, \beta \in A$ e $\alpha \neq \beta$, então $U_{\alpha} \cap U_{\beta} \neq \emptyset$.

Agora, $U_{\alpha} \times V_{\alpha} \cap U_{\beta} \times V_{\beta}=\emptyset$, $\operatorname{logo} V_{\alpha} \cap V_{\beta}=\emptyset$. Assim, $\left\{V_{\alpha}: \alpha \in A\right\}$ é uma família de abertos não vazios e disjuntos de $Y$, uma contradição.

Pelo Teorema 2.9, temos que a resposta afirmativa para a Questão 2.1 é consistente com $Z F C$. Mencionamos ainda o seguinte resultado:

Teorema 2.10 (F.Galvin). Assumindo CH, existem dois espaços compactos Hausdorff com a propriedade c.c.c tal que o produto entre eles não tem a propriedade c.c.c.

Demonstração. Ver [9], Teorema 3.3, página 38.

Concluímos portanto que a resposta a Questão 2.1 é independente de ZFC. 


\subsection{Princípio Diamante $\diamond$}

Nesta seção estudaremos as propriedades referentes a conjuntos fechados e ilimitados (clubs) de um cardinal regular. Destacamos nesta seção o Teorema de Fodor, que nos garante que determinadas funções são constantes em um conjunto estacionário.

Após a introdução dos clubs, introduziremos o Princípio Diamante $\diamond$. Esse princípio é um axioma independente de $Z F C$. Será usado no Capítulo 5, onde construiremos uma álgebra de Boole com propriedades interessantes. Na construção da álgebra de Boole, o Princípio $\diamond$ possui um importante papel de reflexão, pois uma dada propriedade da álgebra será refletida em alguma subálgebra.

Iniciaremos com algumas definições.

Definição 2.13. Se $X$ é um conjunto de ordinais e $\alpha>0$ é um ordinal limite, então $\alpha$ é um ponto limite de $X$ se $\sup (X \cap \alpha)=\alpha$.

Definição 2.14. Seja $\kappa$ um cardinal regular não enumerável.

- Um conjunto $C \subset \kappa$ é fechado se, $C$ contém todos seus pontos limites menores que $\kappa$. Observemos que $C$ é fechado nesta definição se, e somente se, $C$ é um conjunto fechado em $\kappa$ com a topologia da ordem.

- Um conjunto $C \subset \kappa$ é um club se $C$ é fechado e ilimitado em $\kappa$.

- Um conjunto $S \subset \kappa$ é estacionário se $S \cap C \neq \emptyset$ para todo fechado ilimitado $C$ de $\kappa$.

Definição 2.15. Seja $\kappa$ um ordinal $e S \subset \kappa$. Dizemos que uma função $f: S \rightarrow \kappa$ é regressiva se $f(\alpha)<\alpha$ para todo $\alpha \in S$.

Teorema 2.11 (Fodor). Se $f$ é uma função regressiva sobre um conjunto estacionário $S \subset \kappa$, então existe um conjunto estacionário $T \subset S$ e existe $\gamma<\kappa$ tal que $f(\alpha)=\gamma$ para todo $\alpha \in T$.

Demonstração. Ver [17], Lema 6.15, página 80.

Introduziremos agora o Princípio Diamante.

Definição 2.16. O Princípio Diamante $\diamond$ é a sentença :

Existe uma sequência $\left\{S_{\alpha}: \alpha<\omega_{1}\right\}$ de subconjuntos de $\omega_{1}$ tal que

- $\forall \alpha<\omega_{1}\left(S_{\alpha} \subset \alpha\right)$.

- Para todo subconjunto $X \subset \omega_{1}$ temos que $\left\{\alpha: X \cap \alpha=S_{\alpha}\right\}$ é um subconjunto estacionário de $\omega_{1}$.

A sequência $\left\{S_{\alpha}: \alpha<\omega_{1}\right\}$ é denominada de sequência diamante.

A consistência do Princípio $\diamond$ é o conteúdo do Teorema 9.3.1 de [2]. Por outro lado, observemos que o Princípio $\diamond$ implica a Hipótese do Contínuo. De fato, seja $X \subset \omega$. Por hipótese, $\{\alpha: X \cap \alpha=$ $\left.S_{\alpha}\right\}$ é estacionário. Em particular, existe $\alpha$ não enumerável tal que $X \cap \alpha=S_{\alpha}$, ou seja, $X=S_{\alpha}$. Assim $\wp(\omega) \subset\left\{S_{\alpha}: \alpha<\omega_{1}\right\}$.

Como a negação da Hipótese de Contínuo é consistente com $Z F C^{4}$, temos que a negação do Princípio $\diamond$ é consistente com $Z F C$.

Concluímos assim que o Princípio $\diamond$ é um axioma independente de $Z F C$.

Para o Capítulo 5, precisamos obter uma consequência do Princípio $\diamond$ para as potências de $\omega_{1}$. Para isso, precisamos antes do seguinte lema:

\footnotetext{
${ }^{4}$ Ver Teorema 9.2.13 de [2].
} 
Lema 2.10. Fixemos $n \in \omega$. Seja $f: \omega_{1} \rightarrow \omega_{1}^{n}$ bijeção. Então

$$
D=\left\{\alpha: f[\alpha]=\alpha^{n}\right\}
$$

é club.

Demonstração. Suponhamos que $f(\alpha)=\left(f^{1}(\alpha), \ldots, f^{n}(\alpha)\right)$, para todo $\alpha<\omega_{1}$.

Afirmação 1. D é fechado.

Demonstração da Afirmação 1. Seja $\lambda$ limite tal que $D \cap \lambda$ é ilimitado em $\lambda$. Então $\lambda=\bigcup_{\alpha \in D \cap \lambda} \alpha$. Logo,

$$
f[\lambda]=\bigcup_{\alpha \in D_{n} \cap \lambda} f[\alpha]=\bigcup_{\alpha \in D_{n} \cap \lambda} \alpha \times \cdots \times \alpha \subset \lambda \times \cdots \times \lambda .
$$

Por outro lado, seja $\left(\gamma_{1}, \ldots, \gamma_{n}\right) \in \lambda \times \cdots \times \lambda$. Por hipótese, existe $\alpha \in D \cap \lambda$ tal que $\gamma_{1}, \ldots, \gamma_{n} \in$ $\alpha$. Logo, $\left(\gamma_{1}, \ldots, \gamma_{n}\right) \in \alpha \times \cdots \times \alpha$. Assim, $f[\lambda]=\lambda \times \cdots \times \lambda$, portanto, $\lambda \in D$.

Afirmação 2. $D$ é ilimitado.

Demonstração da Afirmação 2. Seja $\beta<\omega_{1}$. Definamos $i: \omega_{1} \rightarrow\left[\omega_{1}\right] \leq \omega$ por $i(\alpha)=\{\beta: \beta<\alpha\}=$ $\alpha$.

Por indução nos naturais, vamos construir um elemento em $D$ maior ou igual a $\beta$.

Seja $Z_{0}=\beta$. Supondo definido $Z_{n}$ seja

$$
Z_{n+1}:=\left\{f^{-1}\left(\alpha_{1}, \ldots, \alpha_{n}\right): \alpha_{1}, \ldots, \alpha_{n} \in Z_{n}\right\} \cup \bigcup_{j=1}^{n}\left\{f^{j}(\alpha): \alpha \in Z_{n}\right\} \cup \bigcup\left\{i(\alpha): \alpha \in Z_{n}\right\} .
$$

Definamos agora $Z=\bigcup_{j \in \omega} Z_{j}$. Por construção, $|Z| \leq|\beta| \leq \omega$. Dado $\alpha \in Z$, existe $j \in \omega$ tal que $\alpha \in Z_{j}$ e assim, $i(\alpha)=\alpha \subset Z_{j+1} \subset Z$. Logo, $\alpha \subset Z$. Portanto, $Z$ é ordinal e pela construção já temos que $\beta \leq Z$. Seja $\alpha \in Z$. Então existe $j \in \omega$ tal que $\alpha \in Z_{j}$ e assim, $f^{i}(\alpha) \in Z_{j+1} \subset Z$ para todo $i=1, \ldots, n$. Logo, $f(\alpha) \in Z \times \cdots \times Z$. Concluímos portanto que $f[Z] \subset Z \times \cdots \times Z$.

Por outro lado, seja $\left(\alpha_{1}, \ldots, \alpha_{n}\right) \in Z \times \cdots \times Z$. Então existe $j \in \omega$ tal que $\alpha_{1}, \ldots, \alpha_{n} \in Z_{j}$. Logo, $f^{-1}\left(\alpha_{1}, \ldots, \alpha_{n}\right) \in Z_{j+1} \subset Z$. Assim, $\left(\alpha_{1}, \ldots, \alpha_{n}\right) \in f[Z]$.

Concluímos portanto que $Z \in D$.

Com o lema acima, vamos demonstrar o seguinte resultado, que será importante no Capítulo 5 .

Lema 2.11. (Princípio $\diamond$ ). Para todo $n \in \mathbb{N}$, existe uma sequência $\left\{\Gamma_{\alpha}^{n}: \alpha<\omega_{1}\right\}$ tal que

- $\Gamma_{\alpha}^{n} \subset \alpha^{n}$.

- Para todo $X \subset \omega_{1}^{n}$, temos que

$$
\left\{\alpha: X \cap \alpha^{n}=\Gamma_{\alpha}^{n}\right\}
$$

é estacionário.

Demonstração. Seja $\left(\Gamma_{\alpha}^{1}\right)_{\alpha<\omega_{1}}$ uma sequência diamante em $\omega_{1}$.

Sejam $n>1$ e $f_{n}: \omega_{1} \rightarrow \omega_{1}^{n}$ bijeção. Pelo lema acima

$$
D_{n}=\left\{\alpha: f_{n}[\alpha]=\alpha^{n}\right\}
$$

é club. Definamos para todo $\alpha<\omega_{1}$

$$
\Gamma_{\alpha}^{n}:=f_{n}\left[\Gamma_{\alpha}^{1}\right] \cap \alpha^{n}
$$

Fixemos agora $X \subset \omega_{1}^{n}$. Vamos mostrar que $\left\{\alpha: X \cap \alpha^{n}=\Gamma_{\alpha}^{n}\right\}$ é estacionário em $\omega_{1}$. 
Tomemos $C$ um club em $\omega_{1}$. Então $D_{n} \cap C$ é club em $\omega_{1}$.

Por outro lado, $\left\{\alpha: f_{n}^{-1}[X] \cap \alpha=\Gamma_{\alpha}^{1}\right\}$ é estacionário, pois $\left(\Gamma_{\alpha}^{1}\right)_{\alpha<\omega_{1}}$ é uma sequência diamante em $\omega_{1}$. Logo, existe $\alpha \in D_{n} \cap C$ tal que $f_{n}^{-1}[X] \cap \alpha=\Gamma_{\alpha}^{1}$. Agora como $\alpha \in D_{n}$ temos que $f_{n}[\alpha]=\alpha^{n}$. Logo,

$$
f_{n}\left[\Gamma_{\alpha}^{1}\right]=X \cap \alpha^{n} .
$$

Assim,

$$
\Gamma_{\alpha}^{n}=X \cap \alpha^{n},
$$

e como $\alpha \in C$ segue-se que

$$
C \cap\left\{\alpha: X \cap \alpha^{n}=\Gamma_{\alpha}^{n}\right\} \neq \emptyset
$$




\section{Capítulo 3}

\section{Sistemas biortogonais}

Do estudo das bases de Schauder em espaços de Banach, sabemos que a existência de uma base de Schauder para um espaço nos fornece muitas informações a respeito do espaço. Por exemplo, se $X$ é espaço de Banach que possui base de Schauder, então $X$ é separável e, pelo Teorema 1.8, $X$ é isomorfo a um espaço de Banach de sequências. Assim, um espaço de Banach não separável não pode ter base de Schauder. Em particular, se $K$ é compacto Hausdorff não metrizável, e $C(K)$ é o espaço de Banach das funções contínuas reais definidas em $K$ com a norma do supremo, então $C(K)$ não possui base de Schauder.

Neste capítulo, estudamos uma outra estrutura de espaços de Banach, que são os sistemas biortogonais. Um sistema biortogonal para um espaço de Banach $X$ é uma família em $X \times X^{*}$, onde $X^{*}$ é o espaço dual de $X$, com certas propriedades.

A definição e alguns exemplos de sistemas biortogonais são apresentados na Seção 3.1. Como exemplo, temos que todo espaço de Banach que possui base de Schauder também possui um sistema biortogonal enumerável, ou seja, um sistema biortogonal de tamanho $\omega$.

Na verdade, como veremos na Seção 3.2, todo espaço de Banach separável possui um sistema biortogonal enumerável. Esse é o conteúdo do Teorema de Markushevic 3.1. Para a demonstração do Teorema de Markushevic, usamos um lema auxiliar que tem semelhança com o processo de ortogonalização de Gram-Schmidt. Observemos que ser separável não implica na existência de base de Schauder, como mostrado por P.Enflo em [5].

Na Seção 3.3, estudamos dois teoremas devido a S. Todorcevic sobre a existência de sistemas biortogonais não enumeráveis em espaços de Banach não separáveis da forma $C(K)$. O primeiro teorema, nos fornece propriedades suficientes que um compacto Hausdorff $K$ deve satisfazer de tal modo que o respectivo espaço de Banach $C(K)$ possua sistemas biortogonais não enumeráveis. Por exemplo, se $K$ é um compacto não separável, teremos que $C(K)$ é um espaço de Banach não separável que possui sistemsa biortogonais não enumeráveis. Assim, obtemos exemplos de espaços de Banach não separáveis que admitem sistemas biortogonais não enumeráveis, partindo de uma classe de espaços compactos Hausdorff. O segundo teorema, nos garante que todo espaço de Banach da forma $C(K)$ não separável possui um sistema biortogonal não enumerável, supondo o Axioma de Martin. Com este último teorema, temos que é consistente com $Z F C$ que todo espaço de Banach não separável da forma $C(K)$ possui sistemas biortogonais não enumeráveis. Em [25], S. Todorcevic provou que é consistente com $Z F C$ que todo espaço de Banach não separável possui sistemas biortogonais não enumeráveis.

Na Seção 3.4 estudamos um teorema que dá condições suficientes que um espaço compacto Hausdorff $K$ deve satisfazer para que o correspondente espaço de Banach $C(K)$ possua apenas sistemas biortogonais enumeráveis. Assim, relacionamos a existência de um espaço de Banach não separável que não possui sistemas biortogonais não enumeráveis com a existência de um espaço compacto satisfazendo certas propriedades. Pelos resultados da seção anterior, é claro que tal exemplo só poderá ser um exemplo consistente. Construiremos um tal exemplo no Capítulo 5, assumindo o Princípio $\diamond$. Os resultados estudados na Seção 3.4 estão contidos no artigo [21].

$\mathrm{Na}$ última seção, estudamos uma aplicação de sistemas biortogonais. Na verdade, considera- 
remos uma versão mais fraca de sistemas biortogonais, os chamados sistemas semibiortogonais e, estudamos a relação dos sistemas semibiortogonais com os conjuntos suportes em espaços de Banach. Destacamos nesta seção o Teorema 3.13 de S. Todorcevic. A demonstração deste teorema será a base para a demonstração de um resultado original contido no Capítulo 4.

\subsection{Definições e exemplos}

Iniciamos esta seção com a definição de sistema biortogonal. Em seguida, estudamos alguns exemplos de sistemas biortogonais, em especial, estudamos a existência de sistemas biortogonais em espaços de Banach da forma $C(K)$ em relação à existência de famílias de abertos dois a dois disjuntos em $K$.

Concluímos a seção com o estudo do comportamento de sistemas biortogonais em relação a subespaços e aplicações lineares.

Definição 3.1. Seja $X$ um espaço de Banach. Seja $\Gamma$ um conjunto não vazio. Uma família $\left\{\left(x_{\gamma}, x_{\gamma}^{*}\right)\right\}_{\gamma \in \Gamma}$ de pares em $X \times X^{*}$ é um sistema biortogonal em $X \times X^{*}$ se

$$
\forall \alpha, \beta \in \Gamma\left(x_{\beta}^{*}\left(x_{\alpha}\right)=\delta_{\alpha, \beta}\right),
$$

onde

$$
\delta_{\alpha \beta}=\left\{\begin{array}{lll}
1, & \text { se } & \alpha=\beta \\
0, & \text { se } & \alpha \neq \beta
\end{array}\right.
$$

Por simplicidade de notação, indicaremos um sistema biortogonal por $\left(x_{\gamma}, x_{\gamma}^{*}\right)_{\gamma \in \Gamma}$.

Observação 3.1. Se $\left(x_{\gamma}, x_{\gamma}^{*}\right)_{\gamma \in \Gamma}$ é um sistema biortogonal em $X \times X^{*}$, então $\left\{x_{\gamma}: \gamma \in \Gamma\right\}$ é um subespaço discreto de $X$. Assim, se $X$ tem densidade $\kappa$, concluímos que $|\Gamma| \leq \kappa{ }^{1}$

Definição 3.2. Seja $\left(x_{\gamma}, x_{\gamma}^{*}\right)_{\gamma \in \Gamma}$ um sistema biortogonal em $X \times X^{*}$. Dizemos que o sistema biortogonal é:

- fundamental se $\overline{\operatorname{span}}\|\cdot\|\left\{x_{\gamma}: \gamma \in \Gamma\right\}=X$,

- total se $\overline{\operatorname{span}}^{\omega^{*}}\left\{x_{\gamma}^{*}: \gamma \in \Gamma\right\}=X^{*}$,

- limitado por uma constante $k$ se $\forall \gamma \in \Gamma\left(\left\|x_{\gamma}\right\| \cdot\left\|x_{\gamma}^{*}\right\| \leq k\right)$.

Definição 3.3 (Base de Markushevich). Uma base de Markushevich para um espaço de Banach X (ou $M$-base para $X$ ) é um sistema biortogonal $\left(x_{\gamma}, x_{\gamma}^{*}\right)_{\gamma \in \Gamma}$ em $X \times X^{*}$ que é fundamental e total.

Vamos estudar agora alguns exemplos de espaços que possuem sistemas biortogonais.

Começamos com os espaços de dimensão finita.

Exemplo 3.1. Consideremos o espaço $X=\mathbb{R}^{n}$. Seja $\left\{e_{1}, \ldots, e_{n}\right\}$ uma base para $X$ e seja $\left\{f_{1}, \ldots, f_{n}\right\}$ a base dual para $X^{*}$. Então $\left(e_{i}, f_{i}\right)_{1 \leq i \leq n}$ é um sistema biortogonal em $X \times X^{*}$ que é uma $M$-base para $\mathbb{R}^{n}$. Temos ainda que tal sistema é limitado por 1 . Observemos que o mesmo vale para qualquer espaço de dimensão finita.

O exemplo acima é um caso particular do seguinte exemplo:

Exemplo 3.2. Seja $X$ um espaço de Banach com base de Schauder $\left(e_{n}\right)_{n \in \mathbb{N}}$. Do Capítulo 1, Seção 1.1.3, vimos que se $\left(f_{n}\right)_{n \in \mathbb{N}}$ em $X^{*}$ é a sequência de funcionais coordenados correspondentes, então $\left(e_{n}, f_{n}\right)_{n \in \mathbb{N}}$ é um sistema biortogonal em $X \times X^{*}$.

Esse sistema é uma M-base e é $2 c$ limitado, onde c é a constante da base.

\footnotetext{
${ }^{1}$ Para espaços métricos, a densidade hereditária é sempre igual a densidade do espaço. Ver [11], Teorema 8.1, página 32.
} 
Estudaremos agora alguns exemplos em espaços de Banach da forma $C(K)$. Começamos com a seguinte proposição:

Proposição 3.1. Seja K um espaço compacto Hausdorff infinito. Suponhamos que K possua uma família $\left\{O_{\alpha}: \alpha<\kappa\right\}$ de abertos dois a dois disjuntos e não vazios. Então $C(K)$ possui um sistema biortogonal de tamanho $\kappa$.

Demonstração. Consideremos a coleção de abertos $\left\{O_{\alpha}: \alpha<\kappa\right\}$ não vazios e dois a dois disjuntos. Para cada $\alpha<\kappa$, escolhemos um elemento $x_{\alpha} \in O_{\alpha}$ e uma função contínua $f_{\alpha}: K \rightarrow[0,1]$ tal que

$$
f_{\alpha}\left(x_{\alpha}\right)=1 \text { e } \forall x \in K \backslash O_{\alpha}\left(f_{\alpha}(x)=0\right) .
$$

Tais funções existem pelo Lema de Urysohn. Por construção, $\left(f_{\alpha}, \delta_{x_{\alpha}}\right)_{\alpha<\kappa}$ é um sistema biortogonal em $C(K) \times C(K)^{*}$. Tal sistema é 1-limitado, mas em geral não é uma M-base.

Exemplo 3.3. Seja $K$ um compacto Hausdorff infinito. Então $C(K)$ possui um sistema biortogonal enumerável.

Demonstração. Como $K$ é compacto Hausdorff infinito, existe uma família $\left\{O_{n}: n<\omega\right\}$ de abertos dois a dois disjuntos e não vazios em $K$. Pela Proposição 3.1, segue-se o resultado.

Lembremos que, uma família $\mathcal{F}$ em uma álgebra de Boole $A$ é uma família disjunta se $a \wedge b=0$ para todos $a, b \in \mathcal{F}$ distintos.

Proposição 3.2. Seja A uma álgebra de Boole. Se A possui uma família disjunta de cardinalidade $\kappa$, então $C(S(A))$ possui um sistema biortogonal de tamanho $\kappa$.

Demonstração. Seja $\left\{a_{\alpha}: \alpha<\kappa\right\}$ uma família disjunta de cardinalidade $\kappa$ em $A$. Então $\left\{s\left(a_{\alpha}\right)\right.$ : $\alpha<\kappa\}$ é uma família de abertos dois a dois disjuntos e não vazios em $S(A)$. Pela Proposição 3.1, segue-se que $C(S(A))$ possui um sistema biortogonal de tamanho $\kappa$.

Exemplo 3.4. $l_{\infty} / c_{0}$ possui um sistema biortogonal de tamanho $\mathfrak{c}$.

Demonstração. Temos que $l_{\infty} / c_{0}=C\left(\omega^{*}\right)$ onde $\omega^{*}$ é o espaço de Stone da álgebra $\wp(\mathbb{N}) / f i n$.

Sabemos que $\wp(\mathbb{N}) /$ fin possui uma família disjunta de cardinalidade $\mathfrak{c}$. Assim, pela Proposição 3.2 concluímos que $l_{\infty} / c_{0}=C\left(\omega^{*}\right)$ possui sistema biortogonal de tamanho $\mathfrak{c}$.

Estudaremos agora a existência de sistemas biortogonais assumindo a existência de sistemas biortogonais para subespaços. Nesta direção temos a seguinte proposição:

Proposição 3.3. Sejam $X$ um espaço de Banach e $Y \subset X$ um subespaço fechado de $Y$. Se $Y$ possui um sistema biortogonal de tamanho $\kappa$, então $X$ também possui um sistema biortogonal de tamanho $\kappa$.

Demonstração. Seja $\left(x_{\alpha}, f_{\alpha}\right)_{\alpha<\kappa}$ um sistema biortogonal em $Y \times Y^{*}$.

Para cada $\alpha<\kappa$, consideremos pelo Teorema de Hahn - Banach 1.1, um funcional $F_{\alpha} \in X^{*}$ tal que $F_{\alpha}$ é extensão de $f_{\alpha}$ e $\left\|F_{\alpha}\right\|=\left\|f_{\alpha}\right\|$.

Sejam $\alpha, \beta \in \kappa$. Então $F_{\alpha}\left(x_{\beta}\right)=f_{\alpha}\left(x_{\beta}\right)=\delta_{\alpha \beta}$ pois $F_{\alpha}$ é extensão de $f_{\alpha}$ e $x_{\beta} \in Y$.

Concluímos portanto que $\left(x_{\alpha}, F_{\alpha}\right)_{\alpha<\kappa}$ é um sistema biortogonal em $X \times X^{*}$.

Estudaremos agora a existência de sistemas biortogonais para um espaço de Banach $X$, supondose a existência de sistemas biortogonais na imagem de $X$.

Proposição 3.4. Sejam $X, Y$ espaços de Banach e $T: X \rightarrow Y$ uma aplicação linear limitada e sobrejetora. Se $Y$ possui um sistema biortogonal de tamanho $\kappa$, então $X$ também possui um sistema biortogonal de tamanho $\kappa$. 
Demonstração. Seja $\left(y_{\alpha}, f_{\alpha}\right)_{\alpha<\kappa}$ um sistema biortogonal em $Y \times Y^{*}$. Como $T$ é sobrejetora, para cada $\alpha<\kappa$ escolhemos um elemento $x_{\alpha} \in X$ tal que $T\left(x_{\alpha}\right)=y_{\alpha}$ e definamos $g_{\alpha}=f_{\alpha} \circ T \in X^{*}$.

Dados $\alpha, \beta \in \kappa$ temos que

$$
g_{\alpha}\left(x_{\beta}\right)=\left(f_{\alpha} \circ T\right)\left(x_{\beta}\right)=f_{\alpha}\left(T\left(x_{\beta}\right)\right)=f_{\alpha}\left(y_{\beta}\right)=\delta_{\alpha \beta} .
$$

Concluímos portanto que $\left(x_{\alpha}, g_{\alpha}\right)_{\alpha<\kappa}$ é um sistema biortogonal em $X \times X^{*}$.

Corolário 3.1. Seja X um espaço de Banach. Se X admite um quociente que possui um sistema biortogonal de tamanho $\kappa$, então $X$ possui um sistema biortogonal de tamanho $\kappa$.

Demonstração. Se $X / Y$ é um quociente de $X$, então a aplicação canônica $\pi: X \rightarrow X / Y$ é contínua e sobrejetora. Assim basta aplicar a Proposição 3.4.

Exemplo 3.5. $l_{\infty}$ possui um sistema biortogonal de tamanho $\mathfrak{c}$.

Demonstração. Sabemos que $l_{\infty} / c_{0}$ possui um sistema biortogonal de tamanho c. Pelo Corolário 3.1 , segue-se que $l_{\infty}$ possui um sistema biortogonal de tamanho $\mathfrak{c}$.

\subsection{Existência de M-bases em espaços de Banach separáveis}

Estudaremos nesta seção o Teorema de Markushevic. Este teorema garante a existência de sistemas biortogonais enumeráveis para todo espaço de Banach separável. O sistema biortogonal enumerável obtido do Teorema de Markushevic é na verdade uma M-base para o espaço.

Observemos que a existência de um sistema biortogonal enumerável para um espaço de Banach separável é também uma consequência do Teorema 1.10 e do Corolário 3.1. Porém, não podemos garantir neste caso que tal sistema biortogonal é uma M-base para o espaço.

Para a demonstração do Teorema de Markushevic, precisamos antes de um resultado similar ao processo de Ortogonalização de Gram-Schmidt. Usaremos a seguinte notação:

Notação 3.1. Seja $X$ um espaço de Banach. Dado $x \in X$ e $x^{*} \in X^{*}$, denotaremos por $\left\langle x, x^{*}\right\rangle$ o valor $x^{*}(x)$, ou seja, $\left\langle x, x^{*}\right\rangle=x^{*}(x)$.

Esta notação facilitará os cálculos e torna mais evidente a analogia entre o próximo lema e o processo de Ortogonalização de Gram-Schmidt.

Lema 3.1. Seja $X$ um espaço de Banach de dimensão infinita. Se $\left\{z_{n}: n \in \mathbb{N}\right\} \subset X$ e $\left\{z_{n}^{*}: n \in\right.$ $\mathbb{N}\} \subset X^{*}$ são tais que $\operatorname{span}\left\{z_{n}: n \in \mathbb{N}\right\}$ e $\operatorname{span}\left\{z_{n}^{*}: n \in \mathbb{N}\right\}$ tenham ambos dimensão infinita $e$

$$
\begin{aligned}
& \left\{z_{n}: n \in \mathbb{N}\right\} \text { separa pontos de } \operatorname{span}\left\{z_{n}^{*}: n \in \mathbb{N}\right\}, \\
& \left\{z_{n}^{*}: n \in \mathbb{N}\right\} \text { separa pontos de } \operatorname{span}\left\{z_{n}: n \in \mathbb{N}\right\} .
\end{aligned}
$$

Então existe um sistema biortogonal $\left(x_{n}, x_{n}^{*}\right)_{n \in \mathbb{N}}$ em $X \times X^{*}$ tal que

$$
\begin{gathered}
\operatorname{span}\left\{x_{n}: n \in \mathbb{N}\right\}=\operatorname{span}\left\{z_{n}: n \in \mathbb{N}\right\} \\
e \\
\operatorname{span}\left\{x_{n}^{*}: n \in \mathbb{N}\right\}=\operatorname{span}\left\{z_{n}^{*}: n \in \mathbb{N}\right\} .
\end{gathered}
$$

Demonstração. Seja $x_{0}=z_{n_{0}}$, onde $n_{0}$ é o primeiro índice $n$ tal que $z_{n} \neq 0$.

Pela Hipótese 3.2 .2 , existe $z_{m_{0}}^{*}$ tal que $\left\langle x_{0}, z_{m_{0}}^{*}\right\rangle \neq 0$.

Definamos então

$$
x_{0}^{*}:=\frac{z_{m_{0}}^{*}}{\left\langle x_{0}, z_{m_{0}}^{*}\right\rangle} .
$$

Temos assim que $\left\langle x_{0}, x_{0}^{*}\right\rangle=1$.

Suponhamos agora que temos definido $\left(x_{i}, x_{i}^{*}\right)_{i<n}$ satisfazendo: 
1. Para todos $i, j<n$, temos que $\left(\left\langle x_{i}, x_{j}^{*}\right\rangle\right)=\delta_{i j}$,

2. $\operatorname{span}\left\{x_{j}: j<n\right\} \subset \operatorname{span}\left\{z_{j}: j \in \mathbb{N}\right\}$ e

3. $\operatorname{span}\left\{x_{j}^{*}: j<n\right\} \subset \operatorname{span}\left\{z_{j}^{*}: j \in \mathbb{N}\right\}$.

- Se $n$ é impar:

Seja $m_{n}$ o menor índice $m$ tal que

$$
z_{m}^{*} \notin \operatorname{span}\left\{x_{i}^{*}: i<n\right\} .
$$

Definamos

$$
x_{n}^{*}:=z_{m_{n}}^{*}-\sum_{i<n}\left\langle x_{i}, z_{m_{n}}^{*}\right\rangle x_{i}^{*} .
$$

Observemos que, se $j<n$ então

$$
\begin{aligned}
\left\langle x_{j}, x_{n}^{*}\right\rangle & =\left\langle x_{j}, z_{m_{n}}^{*}\right\rangle-\sum_{i<n}\left\langle x_{i}, z_{m_{n}}^{*}\right\rangle\left\langle x_{j}, x_{i}^{*}\right\rangle= \\
= & \left\langle x_{j}, z_{m_{n}}^{*}\right\rangle-\sum_{i<n}\left\langle x_{i}, z_{m_{n}}^{*}\right\rangle \delta_{i, j}= \\
& =\left\langle x_{j}, z_{m_{n}}^{*}\right\rangle-\left\langle x_{j}, z_{m_{n}}^{*}\right\rangle=0 .
\end{aligned}
$$

Agora, pela Hipótese 3.2.1, ,omo $x_{n}^{*} \neq 0$, seja $l_{n}$ o menor índice $l$ tal que

$$
\left\langle z_{l}, x_{n}^{*}\right\rangle \neq 0
$$

Definamos

$$
x_{n}:=\frac{z_{l_{n}}-\sum_{i<n}\left\langle z_{l_{n}}, x_{i}^{*}\right\rangle x_{i}}{\left\langle z_{l_{n}}, x_{n}^{*}\right\rangle}
$$

Temos que $\left\langle x_{n}, x_{n}^{*}\right\rangle=1$ e se $j<n$ então

$$
\begin{gathered}
\left\langle x_{n}, x_{j}^{*}\right\rangle=\frac{\left\langle z_{l_{n}}, x_{j}^{*}\right\rangle-\sum_{i<n}\left\langle z_{l_{n}}, x_{i}^{*}\right\rangle\left\langle x_{i}, x_{j}^{*}\right\rangle}{\left\langle z_{l_{n}}, x_{n}^{*}\right\rangle}=\frac{\left\langle z_{l_{n}}, x_{j}^{*}\right\rangle-\sum_{i<n}\left\langle z_{l_{n}}, x_{i}^{*}\right\rangle \delta_{i j}}{\left\langle z_{l_{n}}, x_{n}^{*}\right\rangle}= \\
=\frac{\left\langle z_{l_{n}}, x_{j}^{*}\right\rangle-\left\langle z_{l_{n}}, x_{j}^{*}\right\rangle}{\left\langle z_{l_{n}}, x_{n}^{*}\right\rangle}=0 .
\end{gathered}
$$

- Se $n$ é par:

Seja $l_{n}$ o menor índice $l$ tal que

$$
z_{l} \notin \operatorname{span}\left\{x_{i}: i<n\right\} .
$$

Definamos

$$
x_{n}:=z_{l_{n}}-\sum_{i<n}\left\langle z_{l_{n}}, x_{i}^{*}\right\rangle x_{i} .
$$

Observemos que, se $j<n$ então

$$
\begin{aligned}
\left\langle x_{n}, x_{j}^{*}\right\rangle & =\left\langle z_{l_{n}}, x_{j}^{*}\right\rangle-\sum_{i<n}\left\langle z_{l_{n}}, x_{i}^{*}\right\rangle\left\langle x_{i}, x_{j}^{*}\right\rangle= \\
= & \left\langle z_{l_{n}}, x_{j}^{*}\right\rangle-\sum_{i<n}\left\langle z_{l_{n}}, x_{i}^{*}\right\rangle \delta_{i j}=
\end{aligned}
$$




$$
=\left\langle z_{l_{n}}, x_{j}^{*}\right\rangle-\left\langle z_{l_{n}}, x_{j}^{*}\right\rangle=0 .
$$

Agora, pela Hipotese 3.2.2, como $x_{n} \neq 0$, seja $m_{n}$ o menor índice $m$ tal que $\left\langle x_{n}, z_{m}\right\rangle \neq 0$.

Definamos

$$
x_{n}^{*}:=\frac{z_{m_{n}}^{*}-\sum_{i<n}\left\langle x_{i}, z_{m_{n}}^{*}\right\rangle x_{i}^{*}}{\left\langle x_{n}, z_{m_{n}}^{*}\right\rangle} .
$$

Temos que $\left\langle x_{n}, x_{n}^{*}\right\rangle=1$ e se $j<n$ então

$$
\begin{gathered}
\left\langle x_{j}, x_{n}^{*}\right\rangle=\frac{\left\langle x_{j}, z_{m_{n}}^{*}\right\rangle-\sum_{i<n}\left\langle x_{i}, z_{m_{n}}^{*}\right\rangle\left\langle x_{j}, x_{i}^{*}\right\rangle}{\left\langle z_{m_{n}}, x_{n}^{*}\right\rangle}=\frac{\left\langle x_{j}, z_{m_{n}}^{*}\right\rangle-\sum_{i<n}\left\langle x_{i}, z_{m_{n}}^{*}\right\rangle \delta_{i j}}{\left\langle z_{m_{n}}, x_{n}^{*}\right\rangle}= \\
=\frac{\left\langle x_{j}, z_{m_{n}}^{*}\right\rangle-\left\langle x_{j}, z_{m_{n}}^{*}\right\rangle}{\left\langle z_{m_{n}}, x_{n}^{*}\right\rangle}=0 .
\end{gathered}
$$

Por construção, temos um sistema biortogonal $\left(x_{n}, x_{n}^{*}\right)_{n \in \mathbb{N}}$ em $X \times X^{*}$.

Vamos verificar que

$$
\begin{aligned}
& \operatorname{span}\left\{x_{n}: n \in \mathbb{N}\right\}=\operatorname{span}\left\{z_{n}: n \in \mathbb{N}\right\}, \\
& \operatorname{span}\left\{x_{n}^{*}: n \in \mathbb{N}\right\}=\operatorname{span}\left\{z_{n}^{*}: n \in \mathbb{N}\right\} .
\end{aligned}
$$

Por construção da família $\left\{x_{n}: n \in \mathbb{N}\right\}$, temos que

$$
\operatorname{span}\left\{x_{n}: n \in \mathbb{N}\right\} \subset \operatorname{span}\left\{z_{n}: n \in \mathbb{N}\right\} .
$$

Suponhamos que exista $n \in \mathbb{N}$ tal que $z_{n} \notin \operatorname{span}\left\{x_{j}: j \in \mathbb{N}\right\}$. Em particular, para todo $j \in \mathbb{N}$ temos que $z_{n} \notin \operatorname{span}\left\{x_{i}: i<2 j\right\}$.

Por outro lado, para todo $j \in \mathbb{N}$, não podemos ter $n=\min \left\{l: z_{l} \notin \operatorname{span}\left\{x_{i}: i<2 j\right\}\right.$, caso contrário, pela Condição 3.2.4, teríamos que $z_{n} \in \operatorname{span}\left\{x_{i}: i \leq 2 j\right\} \subset \operatorname{span}\left\{x_{i}: i<(2+1) j\right\}$, uma contradição.

Assim, para cada $j \in \mathbb{N}$, podemos escolher $l_{j}<n$ tal que

$$
\begin{gathered}
z_{l_{j}} \notin \operatorname{span}\left\{x_{i}: i<2 j\right\} \mathrm{e} \\
z_{l_{j}} \in \operatorname{span}\left\{x_{i}: i \leq 2 j\right\} .
\end{gathered}
$$

Observemos que se $j \neq k$, então $l_{j} \neq l_{k}$. Temos portanto uma aplicação injetora $l: \mathbb{N} \rightarrow n$, o que é uma contradição.

Concluímos portanto que $\forall n\left(z_{n} \in \operatorname{span}\left\{x_{n}: n \in \mathbb{N}\right\}\right)$. Assim

$$
\operatorname{span}\left\{x_{n}: n \in \mathbb{N}\right\}=\operatorname{span}\left\{z_{n}: n \in \mathbb{N}\right\} .
$$

De modo análogo se mostra que $\operatorname{span}\left\{x_{n}^{*}: n \in \mathbb{N}\right\}=\operatorname{span}\left\{z_{n}^{*}: n \in \mathbb{N}\right\}$.

Teorema 3.1 (A. I. Markushevic [20]). Todo espaço de Banach separável possui uma M-base.

Demonstração. Seja $X$ um espaço de Banach separável. Suponhamos que $X$ seja de dimensão infinita. Tomemos $\left\{z_{n}\right\}_{n \in \mathbb{N}}$ denso em $(X,\|\cdot\|)$. Pelo Teorema de Alaoglu 1.4, temos que $\left(B_{X^{*}}, \omega^{*}\right)$ é compacto e, como $X$ é separável, segue-se pela Proposição 1.2 que $\left(B_{X^{*}}, \omega^{*}\right)$ é metrizável. Logo, $\left(B_{X^{*}}, \omega^{*}\right)$ é separável. Seja então $\left\{z_{n}^{*}\right\}_{n \in \mathbb{N}}$ denso em $\left(B_{X^{*}}, \omega^{*}\right)$.

Temos que $\operatorname{span}\left\{z_{n}\right\}_{n \in \mathbb{N}}$ tem dimensão infinita já que $\overline{\operatorname{span}}\|\cdot\|\left\{z_{n}: n \in \omega\right\}=X$ e $X$ tem dimensão infinita por hipótese.

De modo análogo, pela Proposicao 1.3, todo espaço de dimensão finita é $\omega^{*}$ fechado. Concluímos assim que $\operatorname{span}\left\{z_{n}^{*}\right\}_{n \in \mathbb{N}}$ tem dimensão infinita. 
Sejam agora $x \neq y \in \operatorname{span}\left\{z_{n}\right\}_{n \in \mathbb{N}}$. Então existe $f \in B_{X^{*}}$ tal que $f(x) \neq f(y)$. Como $\left\{z_{n}^{*}\right\}_{n}$ é denso em $\left(B_{X^{*}}, \omega^{*}\right)$, existe uma sequência $\left(z_{n_{k}}\right)_{k}$ em $B_{X^{*}}$ tal que $z_{n_{k}} \stackrel{\omega^{*}}{\longrightarrow} f$. Em particular, $z_{n_{k}}(x) \stackrel{k}{\longrightarrow} f(x)$ e $z_{n_{k}}(y) \stackrel{k}{\longrightarrow} f(y)$. Assim deve existir $k \in \mathbb{N}$ tal que $z_{n_{k}}(x) \neq z_{n_{k}}(y)$.

Concluímos assim que $\left\{z_{n}^{*}\right\}_{n}$ separa pontos de $\operatorname{span}\left\{z_{n}\right\}_{n}$. De modo análogo, $\left\{z_{n}\right\}_{n}$ separa pontos de $\operatorname{span}\left\{z_{n}^{*}\right\}_{n}$. Assim, para concluirmos o teorema, basta aplicarmos o Lema 3.1.

Como observado anteriormente, existem espaços de Banach separáveis que não admitem uma base de Schauder, como foi mostrado por P.Enflo em [5]. No caso de sistemas biortogonais, o Teorema 3.1 nos mostra que todo espaço separável admite um sistema biortogonal enumerável. Assim, se $X$ é espaço de Banach separável (ou seja, $X$ tem densidade enumerável), então $X$ admite um sistema biortogonal de tamanho igual a sua densidade ( ou seja, enumerável). Neste ponto, surge a questão de saber se o mesmo é verdadeiro para espaços de Banach de densidade maior que $\omega$, ou seja, se é verdade que todo espaço de Banach possui um sistema biortogonal de tamanho igual a sua densidade. Esse assunto será tratado nas próximas seções.

Uma consequência do Teorema 3.1 é o seguinte corolário:

Corolário 3.2. Todo espaço de Banach de dimensão infinita possui um sistema biortogonal enumerável.

Demonstração. Seja $X$ um espaço de Banach de dimensão infinita. Tomemos $Y$ um subspaço fechado de $X$ separável. Pelo Teorema 3.1, concluímos que $Y$ admite um sistema biortogonal enumerável. Pela Proposição 3.3 segue-se que $X$ admite um sistema biortogonal enumerável.

Encerramos esta seção com um resultado para espaços de Banach reflexivos.

Teorema 3.2. Seja X um espaço de Banach. Então, $X$ é reflexivo se, e somente se, $X$ possui uma M-base.

Demonstração. Ver [10], Teorema 6.1, página 208.

\subsection{Existência de sistemas biortogonais não enumeráveis em espaços $C(K)$}

Nesta seção, vamos estudar dois teoremas de S.Todorcevic sobre sistemas biortogonais em espaços de Banach $C(K)$.

Começamos esta seção estudando uma condição suficiente que um compacto Hausdorff deve satisfazer para que o respectivo espaço de Banach possua um sistema biortogonal não enumerável.

Teorema 3.3 (S. Todorcevic [25]). Seja K um compacto Hausdorff contendo um subespaço não separável. Então $C(K)$ contém um sistema biortogonal não enumerável.

Demonstração. Primeiramente, faremos algumas simplificações.

Afirmação 1: É suficiente supor que K não é separável.

Demonstração da Afirmação 1. Seja $L \subset K$ um subespaço não separável. Suponha que o resultado vale para $\bar{L}$. Então, existe um sistema biortogonal não enumerável em $C(\bar{L}) \times C(\bar{L})^{*}$. Seja $T$ : $C(K) \rightarrow C(\bar{L})$ dada por $T(f)=f_{\mid \bar{L}}$. Temos que $T$ é um operador linear e contínuo. Além disso, $T$ é sobrejetor, pelo Teorema de extensão de Tietze. Concluímos pela Proposição 3.4 que $C(K)$ possui um sistema biortogonal não enumerável.

Pela Afirmação 1, podemos assumir que $K$ não é separável.

Afirmação 2: É suficiente supor que $K$ tem a propriedade c.c.c. 
Demonstração da Afirmação 2. Suponha que $K$ não tenha a propriedade c.c.c. Assim existe $\left\{\theta_{\gamma}, \gamma<\right.$ $\left.\omega_{1}\right\}$ uma família não enumerável de abertos não vazios dois a dois disjuntos. Segue-se da Proposição 3.1 que existe um sistema biortogonal de tamanho $\omega_{1}$ em $C(K) \times C(K)^{*}$.

Pela Afirmação 2, podemos assumir que $K$ tem a propriedade c.c.c.

Vamos construir recursivamente para cada $\alpha<\omega_{1}$, uma função $f_{\alpha}: K \rightarrow[0,1]$ e pontos $x_{\alpha}, y_{\alpha} \in K$ tais que

1. $f_{\alpha}\left(x_{\alpha}\right)=1$ e $f_{\alpha}\left(y_{\alpha}\right)=0$,

2. $f_{\alpha}\left(x_{\beta}\right)=f_{\alpha}\left(y_{\beta}\right)=0$ para $\beta<\alpha$,

3. $f_{\alpha}\left(x_{\beta}\right)=f_{\alpha}\left(y_{\beta}\right)$ para $\alpha<\beta$.

Suponhamos que temos definido $f_{\alpha}, x_{\alpha}$ e $y_{\alpha}$ para todo $\alpha<\beta$ satisfazendo as três condições acima.

Afirmação 3: Existem $x \neq y$ em $K \backslash \overline{\left\{x_{\alpha}, y_{\alpha}: \alpha<\beta\right\}}$ tais que $f_{\alpha}(x)=f_{\alpha}(y)$ para todo $\alpha<\beta$.

Demonstração da Afirmação 3. Suponhamos que a Afirmação não seja verdadeira. Então para todo aberto $B$ de $K$ tal que $\bar{B} \cap \overline{\left\{x_{\alpha}, y_{\alpha}: \alpha<\beta\right\}}=\emptyset$, temos que $B$ é separável. De fato, vamos mostrar que

$$
\mathfrak{B}=\left\{f_{\alpha}^{-1}[(a, b)] \cap B: \alpha<\beta \text { e } a<b \in \mathbb{Q}\right\}
$$

é uma subbase para $B$.

Seja $x \in V \cap B$ com $V$ aberto de $K$. Por hipótese, para todo $y \in \bar{B} \backslash V$ existe $\alpha_{y}<\beta$ e $a_{y}<b_{y} \in \mathbb{Q}$ tais que

$$
x \in f_{\alpha_{y}}^{-1}\left[\left(a_{y}, b_{y}\right)\right] \text { e } y \in f_{\alpha_{y}}^{-1}\left[\mathbb{R} \backslash\left[a_{y}, b_{y}\right]\right] .
$$

Observemos que

$$
\bar{B} \backslash V \subset \bigcup_{y \in \bar{B} \backslash V} f_{\alpha_{y}}^{-1}\left[\mathbb{R} \backslash\left[a_{y}, b_{y}\right]\right]
$$

Como $\bar{B} \backslash V$ é compacto, existem $y_{1}, \ldots, y_{n} \in \bar{B} \backslash V$ tais que $\bar{B} \backslash V \subset \bigcup_{i=1}^{n} f_{\alpha_{y_{i}}}^{-1}\left[\mathbb{R} \backslash\left[a_{y_{i}}, b_{y_{i}}\right]\right]$.

Temos então que $x \in \bigcap_{i=1}^{n}\left(f_{\alpha_{y_{i}}}^{-1}\left[\left(a_{y_{i}}, b_{y_{i}}\right)\right] \cap B\right) \subset V$. Logo, $\mathfrak{B}$ é subbase para $B$. Mostramos assim que $B$ possui uma base de abertos enumerável e, portanto, $B$ é separável.

Seja $\mathbb{U}$ uma família maximal de abertos dois a dois disjuntos tal que se $B \in \mathbb{U}$ então $\bar{B} \cap$ $\overline{\left\{x_{\alpha}, y_{\alpha}: \alpha<\beta\right\}}=\emptyset$. (Família obtida através do Lema de Zorn). Como $K$ tem a propriedade c.c.c, segue-se que $\mathbb{U}$ é enumerável e, portanto, $\bigcup \mathbb{U}$ é um subespaço separável. Logo,

$$
K^{\prime}=(\bigcup \mathbb{U}) \cup\left\{x_{\alpha}, y_{\alpha}: \alpha<\beta\right\}
$$

é separável.

Observemos que, $\overline{K^{\prime}}=K$ pois caso contrário, $B^{\prime}=K \backslash \overline{K^{\prime}}$ é aberto, com $B^{\prime} \cap \overline{\left\{x_{\alpha}, y_{\alpha}: \alpha<\beta\right\}}=$ $\emptyset$ e $B^{\prime}$ é disjunto de $\bigcup \mathbb{U}$, uma contradição com a maximalidade de $\mathbb{U}$. Logo $\overline{K^{\prime}}=K$ e, portanto, $K$ deve ser separável, o que é uma contradição, pois estamos supondo que $K$ não é separável.

Tomemos então $x_{\beta}, y_{\beta} \in K \backslash \overline{\left\{x_{\alpha}, y_{\alpha}: \alpha<\beta\right\}}$ tal que $f_{\alpha}\left(x_{\beta}\right)=f_{\alpha}\left(y_{\beta}\right)$ para todo $\alpha<\beta$ e seja, pelo Lema de Urysohn, $f_{\beta}: K \rightarrow[0,1]$ tal que

$$
f_{\beta}\left(x_{\beta}\right)=1 \text { e } f_{\beta}(x)=0 \text { para todo } x \in\left\{y_{\alpha}\right\} \cup \overline{\left\{x_{\alpha}, y_{\alpha}: \alpha<\beta\right\}} .
$$

Por indução, temos $f_{\alpha}\left(\alpha<\omega_{1}\right)$ e $x_{\alpha}, y_{\alpha}\left(\alpha<\omega_{1}\right)$ satisfazendo 1,2 e 3 . Vejamos que

$$
\left(f_{\alpha}, \delta_{x_{\alpha}}-\delta_{y_{\alpha}}\right)_{\alpha<\omega_{1}}
$$

é um sistema biortogonal. 
Temos que para todo $\alpha<\omega_{1}, \delta_{x_{\alpha}}-\delta_{y_{\alpha}} \in C(K)^{*} \mathrm{e}$

$$
\begin{gathered}
\left(\delta_{x_{\alpha}}-\delta_{y_{\alpha}}\right)\left(f_{\alpha}\right)=f_{\alpha}\left(x_{\alpha}\right)-f_{\alpha}\left(y_{\alpha}\right)=f_{\alpha}\left(x_{\alpha}\right)=1 \mathrm{e} \\
\forall \beta \neq \alpha\left[\left(\delta_{x_{\alpha}}-\delta_{y_{\alpha}}\right)\left(f_{\beta}\right)=f_{\beta}\left(x_{\alpha}\right)-f_{\beta}\left(y_{\alpha}\right)=0\right] .
\end{gathered}
$$

Logo $\left(f_{\alpha}, \delta_{x_{\alpha}}-\delta_{y_{\alpha}}\right)_{\alpha<\omega_{1}}$ é um sistema biortogonal em $C(K)$.

O Teorema acima nos dá condições suficientes que um compacto Hausdorff $K$ deve satisfazer de tal modo que o espaço de Banach $C(K)$ admita um sistema biortogonal não enumerável. Em particular, para todo espaço compacto Hausdorff não separável $K$, o espaço de Banach $C(K)$ é um espaço de Banach não separável que possui um sistema biortogonal não enumerável.

Aplicaremos agora o Axioma de Martin para estudarmos o Teorema 3.6 que garante que todo espaço de Banach da forma $C(K)$ não separável, admite um sistema biortogonal não enumerável. Primeiramente, precisamos de algumas definições.

Definição 3.4. Seja X um espaço topológico.

- Dizemos que X é hereditariamente separável se todo subespaço de X é separável.

- Uma sequência $\left\{x_{\alpha}, \alpha<\kappa\right\}$ em $X$ é uma sequência livre se, para todo $\alpha<\kappa$, temos que $\overline{\left\{x_{\beta}: \beta \leq \alpha\right\}} \cap \overline{\left\{x_{\beta}: \beta>\alpha\right\}}=\emptyset$.

- Dizemos que $X$ possui tightness enumerável se, para todo $y \in \bar{Y}$ com $Y \subset X$, existe $Y^{\prime} \subset Y$ enumerável tal que $y \in \overline{Y^{\prime}}$.

Observemos que, se $X$ é hereditariamente separável, então $X$ possui tightness enumerável.

Teorema 3.4. Se $X$ possui tightness enumerável, então $X$ não possui uma sequência livre de tamanho $\omega_{1}$.

Demonstração. Ver [11], Teorema 7.8, página 27.

Teorema 3.5. Se X possui tightness enumerável, então todo potência finita de X possui tightness enumerável.

Demonstração. Ver [19].

Com os teoremas acima, obtemos o seguinte corolário que será usado na demonstração do Teorema 3.6.

Corolário 3.3. Se K é um espaço compacto Hausdorff hereditariamente separável, então não existe sequência livre de tamanho $\omega_{1}$ em nenhuma potência finita de $X$.

Demonstração. Como $K$ é hereditariamente separável, temos que $K$ possui tightness enumerável. Pelo Teorema 3.5, toda potência finita de $K$ possui tightness enumerável. Assim, pelo Teorema 3.4, nenhuma potência finita de $K$ possui uma sequência livre de tamanho $\omega_{1}$.

Estamos agora em condições de estudar o seguinte Teorema:

Teorema 3.6 (S. Todorcevic [25]). $(M A+\neg C H)$. Se $C(K)$ não é separável, então existe um sistema biortogonal não enumerável em $C(K) \times C(K)^{*}$.

Demonstração. Faremos aqui apenas a demonstração do caso em que $K=S(A)$, onde $A$ é uma álgebra de Boole de tamanho $\omega_{1}$. Para a demonstração do caso geral, indicamos o artigo [25] de S. Todorcevic.

Pelo Teorema 3.3, podemos assumir que $K$ é hereditariamente separável. Como $|A|=\omega_{1}$, podemos escrever $A$ como a união de uma família estritamente crescente $\left(A_{\alpha}\right)_{\alpha<\omega_{1}}$ de subálgebras de Boole de $A$. Pelo Lema 1.2, para cada $\alpha<\omega_{1}$, se $a_{\alpha} \in A_{\alpha+1} \backslash A_{\alpha}$, existem ultrafiltros distintos $p_{\alpha}, q_{\alpha}$ de $A$ tais que $\chi_{s\left(a_{\alpha}\right)}\left(p_{\alpha}\right) \neq \chi_{s\left(a_{\alpha}\right)}\left(q_{\alpha}\right)$ e $\chi_{s(b)}\left(p_{\alpha}\right) \neq \chi_{s(b)}\left(q_{\alpha}\right)$ para todo $b \in A_{\alpha}$. Definamos $x_{\alpha}=p_{\alpha}, y_{\alpha}=q_{\alpha}$ e $f_{\alpha}=\chi_{s\left(a_{\alpha}\right)}$. Assim, 
- $f_{\alpha}\left(x_{\alpha}\right) \neq f_{\alpha}\left(y_{\alpha}\right)$ para todo $\alpha<\omega_{1}$ e

- $f_{\beta}\left(x_{\alpha}\right)=f_{\beta}\left(x_{\alpha}\right)$ para todos $\beta<\alpha<\omega_{1}$.

Consideremos a ordem parcial $\mathbb{P}=\left(\left[\omega_{1}\right]^{<\omega}, \leq\right)$ onde, $p \leq q$ se, e somente se, $q \subset p$ e

$$
\forall \gamma \in q \quad \forall \delta \in p \backslash q\left(f_{\gamma}\left(x_{\delta}\right)=f_{\gamma}\left(y_{\delta}\right)\right) .
$$

\section{Afirmação 1. $\mathbb{P}$ tem a c.c.c.}

Demonstração da Afirmação 1. Seja $\left\{p_{\alpha}: \alpha<\omega_{1}\right\}$ uma família não enumerável em $\mathbb{P}$. Vamos mostrar que existem dois elementos em $\left\{p_{\alpha}: \alpha<\omega_{1}\right\}$ que são compatíveis.

Pelo Lema do $\Delta$-sistema na versão contida no Teorema 2.3 , podemos assumir que existe um subconjunto finito $\Delta \subset \omega_{1}$ e existe $k \in \mathbb{N}$ tais que

1) $p_{\alpha} \cap p_{\beta}=\Delta$ para distintos $\alpha, \beta \in \omega_{1}$,

2) $\Delta<p_{\alpha} \backslash \Delta<p_{\beta} \backslash \Delta$ para $\alpha<\beta<\omega_{1}$,

3) $\left|p_{\alpha} \backslash \Delta\right|=k$ e $p_{\alpha} \backslash \Delta=\left(\xi_{1}(\alpha), \ldots, \xi_{k}(\alpha)\right)$ para todo $\alpha<\omega_{1}$.

Definamos para todos pares $\alpha<\beta$ o elemento $p_{\alpha \beta}=p_{\alpha} \cup p_{\beta}$. Observamos primeiramente que se $\alpha<\beta$, então $p_{\alpha} \subset p_{\alpha \beta}$ e $p_{\alpha \beta} \backslash p_{\alpha}=p_{\beta} \backslash \Delta$ pela Propriedade 1). Ainda, pela Propriedade 2), temos que $\Delta<p_{\alpha} \backslash \Delta<p_{\beta} \backslash \Delta \operatorname{logo}, f_{\gamma}\left(x_{\delta}\right)=f_{\gamma}\left(y_{\delta}\right)$ para todo $\gamma \in p_{\alpha} \backslash \Delta$ e para todo $\delta \in p_{\beta} \backslash \Delta$. Assim, vale que

$$
\forall \gamma \in p_{\alpha} \quad \forall \delta \in p_{\alpha \beta} \backslash p_{\alpha} \quad\left(f_{\gamma}\left(x_{\delta}\right)=f_{\gamma}\left(y_{\delta}\right)\right) .
$$

Logo $p_{\alpha \beta} \leq p_{\alpha}$. Portanto, para concluirmos a demonstração da afirmação, basta encontrarmos $\alpha<\beta<\omega_{1}$ tais que $p_{\alpha \beta}<p_{\beta}$, pois assim, $p_{\alpha \beta}$ será uma extensão comum de $p_{\alpha}$ e $p_{\beta}$. Suponhamos por absurdo que para todos $\alpha<\beta<\omega_{1}$, temos que $p_{\alpha \beta} \not \leq p_{\beta}$. Assim, para todos $\alpha<\beta<\omega_{1}$, devem existir $\gamma \in p_{\beta} \backslash \Delta$ e $\delta \in p_{\alpha} \backslash \Delta$ tais que $f_{\gamma}\left(x_{\delta}\right) \neq f_{\gamma}\left(y_{\delta}\right)$.

Para cada $\beta<\omega_{1}$, consideremos

$$
w_{\beta}=\left(x_{\xi_{1}(\beta)}, y_{\xi_{1}(\beta)}, \ldots, x_{\xi_{k}(\beta)}, y_{\xi_{k}(\beta)}\right) \in K^{2 k}
$$

$\mathrm{e}$

$$
W_{\beta}=\bigcup_{1 \leq i \leq k} W_{\beta, i}
$$

onde

$$
W_{\beta, i}=\bigcup_{1 \leq j \leq k} K^{2} \times \cdots \times\left\{s\left(a_{\xi_{i}(\beta)}\right) \times s\left(-a_{\xi_{i}(\beta)}\right) \cup s\left(-a_{\xi_{i}(\beta)}\right) \times s\left(a_{\xi_{i}(\beta)}\right)\right\} \times \cdots \times K^{2}
$$

Temos que cada $W_{\beta}$ é um clopen de $K^{2 k}$. Observemos que dado $\left(x_{1}, y_{1}, \ldots, x_{k}, y_{k}\right) \in K^{2 k}$ tal que existem $i, j=1, \ldots, k$ satisfazendo $f_{\xi_{i}(\beta)}\left(x_{j}\right)=\chi_{a_{\xi_{i}(\beta)}}\left(x_{j}\right) \neq \chi_{a_{\xi_{i}(\beta)}}\left(y_{j}\right)=f_{\xi_{i}(\beta)}\left(y_{j}\right)$, temos que $\left(x_{1}, y_{1}, \ldots, x_{k}, y_{k}\right) \in W_{\beta, i} \subset W_{\beta}$. Assim, para todo $\alpha \leq \beta$, temos que $w_{\alpha} \in W_{\beta}$.

Por outro lado, se $\alpha>\beta$, pela escolha das famílias $\left(f_{\alpha}\right)_{\alpha}$ e $\left(x_{\alpha}, y_{\alpha}\right)_{\alpha}$, temos que $f_{\xi_{i}(\beta)}\left(x_{\xi_{j}(\alpha)}\right)=$ $f_{\xi_{i}(\beta)}\left(y_{\xi_{j}(\alpha)}\right)$ para todos $i, j=1, \ldots, k$, pois $\xi_{j}(\alpha)>\xi_{i}(\beta)$ para todos $i, j=1, \ldots, k$. Concluímos assim que $w_{\alpha} \notin W_{\beta}$ para $\alpha>\beta$.

Pelas observações acimas, temos uma família de pontos $\left(w_{\alpha}\right)_{\alpha<\omega_{1}}$ em $K^{2 k}$ e uma família de clopens $\left(W_{\alpha}\right)_{\alpha<\omega_{1}}$ satisfazendo

- para todo $\alpha \leq \beta$, temos que $w_{\alpha} \in W_{\beta}$,

- para todo $\alpha>\beta$, temos que $w_{\alpha} \notin W_{\beta}$.

Assim, $\left(w_{\alpha}\right)_{\alpha<\omega_{1}}$ é uma sequência livre de tamanho $\omega_{1}$ em $K^{2 k}$. Porém, $K$ é um compacto Hausdorff hereditariamente separável, o que contradiz o Corolário 3.3. 
Para cada $\alpha<\omega_{1}$, definamos $D_{\alpha}:=\left\{p \in \mathbb{P}: p \cap\left[\alpha, \omega_{1}\right) \neq \emptyset\right\}$.

Afirmação 2. $D_{\alpha}$ é denso em $\mathbb{P}$ para cada $\alpha<\omega_{1}$.

Demonstração da Afirmação 2. Seja $p \in \mathbb{P}$ e suponhamos que $p \cap\left[\alpha, \omega_{1}\right)=\emptyset$. Definamos $q=p \cup\{\alpha\}$. Então $p \subset q$ e para todo $\gamma \in p$, temos que $\gamma<\alpha$ e, portanto, $f_{\gamma}\left(x_{\alpha}\right)=f_{\gamma}\left(y_{\alpha}\right)$. Assim, vale que

$$
\forall \gamma \in p \quad \forall \delta \in q \backslash p=\{\alpha\} \quad\left(f_{\gamma}\left(x_{\delta}\right)=f_{\gamma}\left(y_{\delta}\right)\right) .
$$

Logo, $q$ é uma extensão de $p$ em $D_{\alpha}$.

Pelo Axioma de Martin, existe $G$ um filtro em $\mathbb{P}$ que intersecta cada elemento em $\left\{D_{\alpha}: \alpha<\omega_{1}\right\}$. Definamos $A:=\bigcup G$. Como $G \cap D_{\alpha} \neq \emptyset$ para cada $\alpha<\omega_{1}$, temos que $A$ é não enumerável.

Para cada $\beta \in A$, fixemos $q_{\beta} \in G$ tal que $\beta \in q_{\beta}$.

Afirmação 3. Seja $\beta \in A$. Para todo $\alpha \in A \backslash\left(q_{\beta} \cap \beta\right)$ temos que, $f_{\beta}\left(x_{\alpha}\right)=f_{\beta}\left(y_{\alpha}\right)$.

Demonstração da Afirmação 3. Seja $\alpha \in A \backslash\left(q_{\beta} \cap \beta\right)$ e $q \in G$ tal que $\alpha \in q$. Como $G$ é filtro, existe $r \in G$ tal que $r \leq q, q_{\beta}$. Em particular, $r \leq q_{\beta}$ e, portanto, vale que

$$
\forall \gamma \in r \backslash q_{\beta} \quad\left(f_{\beta}\left(x_{\gamma}\right)=f_{\beta}\left(y_{\gamma}\right)\right) .
$$

Se $\alpha>\beta$, então já temos que $f_{\beta}\left(x_{\alpha}\right)=f_{\beta}\left(y_{\beta}\right)$. Suponhamos então que $\alpha<\beta$. Como $\alpha \notin q_{\beta} \cap \beta$, segue que $\alpha \notin q_{\beta}$. Logo, $\alpha \in r \backslash q_{\beta}$. Portanto, de 3.3.1, obtemos que $f_{\beta}\left(x_{\alpha}\right)=f_{\beta}\left(y_{\alpha}\right)$.

Seja $A=\left\{\gamma_{\alpha}: \alpha<\omega_{1}\right\}$ uma enumeração de $A$. Para cada $\omega<\delta<\omega_{1}$, seja $\phi_{\delta}<\delta$ tal que $\gamma_{\phi_{\delta}}=\sup \left(q_{\gamma_{\delta}} \cap \gamma_{\delta}\right)$. Pelo Lema de Fodor 2.11, existe $\Gamma \subset\left[\omega, \omega_{1}\right)$ estacionário e $\phi<\omega_{1}$ tal que $\phi_{\delta}=\phi$ para todo $\delta \in \Gamma$. Temos assim que $\gamma_{\phi}=\sup \left(q_{\gamma_{\delta}} \cap \gamma_{\delta}\right)$ para todo $\delta \in \Gamma$. Sejam $\alpha, \beta \in \Gamma$. Se $\beta<\alpha$, então $\gamma_{\beta}<\gamma_{\alpha}$ e, portanto, $f_{\gamma_{\beta}}\left(x_{\gamma_{\alpha}}\right)=f_{\gamma_{\beta}}\left(x_{\gamma_{\alpha}}\right)$. Por outro lado, se $\alpha<\beta$, então $\gamma_{\phi}<\gamma_{\alpha}<\gamma_{\beta}$. Pela escolha de $\phi$, temos que $q_{\gamma_{\beta}} \cap \gamma_{\beta} \subset\left[0, \gamma_{\phi}\right]$. Assim, $\gamma_{\alpha} \in A \backslash\left[0, \gamma_{\phi}\right] \subset A \backslash\left(q_{\gamma_{\beta}} \cap \gamma_{\beta}\right)$. Logo, pela Afirmação 3 , temos que $f_{\gamma_{\beta}}\left(x_{\gamma_{\alpha}}\right)=f_{\gamma_{\beta}}\left(y_{\gamma_{\alpha}}\right)$. Concluímos portanto que

$$
\left(f_{\gamma_{\alpha}}, \delta_{x_{\gamma_{\alpha}}}-\delta_{y_{\gamma_{\alpha}}}\right)_{\alpha \in \Gamma}
$$

é um sistema biortogonal não enumerável em $C(K) \times C(K)^{*}$.

Pelo Teorema 3.6, é consistente com $Z F C$ que todo espaço de Banach não separável da forma $C(K)$ possui um sistema biortogonal não enumerável. Mencionamos ainda o seguinte teorema devido a S.Todorcevic:

Teorema 3.7 (S. Todorcevic [25]). (MM) Seja X um espaço de densidade $\omega_{1}$. Então, X possui um sistema biortogonal fundamental. Em particular, todo espaço de Banach não separável possui um sistema biortogonal não enumerável.

Demonstração. Ver [25], Corolário 7, página 706.

O Máximo de Martin $(M M)$ é uma versão mais forte que $M A$ e é consistente com $Z F C$. Indicamos [8] para uma formulação precisa de $(M M)$ e algumas consequências.

Concluímos portanto que é consistente com $Z F C$ que todo espaço de Banach não separável possui um sistema biortogonal não enumerável. Na próxima seção, estudaremos algumas condições suficientes que um espaço compacto $K$ deve satisfazer de tal modo que o espaço de Banach $C(K)$ não possua sistemas biortogonais não enumeráveis e no Capítulo 5 construiremos com o Princípio $\diamond$, um compacto satisfazendo tais propriedades. Deste modo, temos que a questão de saber se todo espaço de Banach não separável possui um sistema biortogonal não enumerável é independente de $Z F C$. 


\subsection{Não existência de sistemas biortogonais não enumeráveis em espaços $C(K)$}

O objetivo desta seção é estudar algumas propriedades necessárias que um espaço compacto Hausdorff não metrizável $K$ deve satisfazer para que o respectivo espaço de Banach $C(K)$ possua apenas sistemas biortogonais enumeráveis. Deste modo, a construção de um espaço de Banach não separável que não possua sistemas biortogonais não enumerável se reduz à construção de um espaço compacto Hausdorff satisfazendo certas propriedades.

Primeiramente, veremos que se um espaço de Banach $\mathrm{X}$ possui um sistema biortogonal não enumerável, então o espaço $(X, \omega)$ admite um subespaço discreto não enumerável. Este é o conteúdo da próxima proposição:

Proposição 3.5. Seja $X$ um espaço de Banach. Se existe um sistema biortogonal de tamanho $\kappa$ em $X \times X^{*}$ então, existe um subespaço discreto de cardinalidade $\kappa$ em $(X, \omega)$.

Demonstração. Seja $\left(x_{\alpha}, x_{\alpha}^{*}\right)_{\alpha<\kappa}$ um sistema biortogonal em $X \times X^{*}$. Consideremos o conjunto $Y=\left\{x_{\alpha}: \alpha<\kappa\right\}$ em $X$. Para cada $\alpha<\kappa$, pela definição de sistema biortogonal, temos que $U\left(x_{\alpha}, 1, x_{\alpha}^{*}\right)$ é uma vizinhança fraca de $x_{\alpha}$ tal que $U\left(x_{\alpha}, 1, x_{\alpha}^{*}\right) \cap Y=\left\{x_{\alpha}\right\}$. Logo $Y$ é um subespaço discreto de $(X, \omega)$.

Pela Proposição 3.5, dado um espaço compacto Hausdorff $K$, se $C(K)$ não possui subespaços discretos não enumeráveis (na topologia fraca), então $C(K)$ não possui sistemas biortogonais não enumeráveis. Assim, obteremos algumas propriedades que um espaço compacto $K$ deve satisfazer, de modo que todo subespaço discreto de $\left(C(K)^{*}, \omega\right)$ seja enumerável.

Definição 3.5. Um espaço topológico $X$ é hereditariamente separável se, todo subespaço $Y \subset X$ é separável.

Lema 3.2. Seja $X=\prod_{n<\omega} X_{n}$ um espaço topológico produto tal que todo subproduto finito é hereditariamente separável. Então $X$ é hereditariamente separável.

Demonstração. Seja $Y \subset X$ um subespaço. Para cada $F \subset \omega$ finito, temos por hipótese que $\pi_{F}[Y] \subset \prod_{n \in F} X_{n}$ é separável, onde $\pi_{F}: X \rightarrow \prod_{n \in F} X_{n}$ é a projeção de $X$ sobre $\prod_{n \in F} X_{n}$. Seja então $D_{F}^{0} \subset \pi_{F}[Y]$ um subconjunto denso e tomemos $D_{F} \subset Y$ enumerável tal que $\pi_{F}\left[D_{F}\right]=D_{F}^{0}$.

Consideremos $D=\bigcup\left\{D_{F}: F \in[\omega]^{<\omega}\right\}$. Temos que $D$ é enumerável e, além disso, $D$ é denso em $Y$. De fato, seja $Y \cap \prod_{i<\omega} B_{i}$ aberto em $Y$, onde $B_{i}=X_{i}$ para $i \in \omega \backslash F$ para $F \subset \omega$ finito e $B_{i}$ é aberto em $X_{i}$ para $i \in F$. Então

$$
\pi_{F}\left[Y \cap \prod_{i<\omega} B_{i}\right]=\pi_{F}[Y] \cap \prod_{i \in F} B_{i}
$$

é aberto em $\pi_{F}[Y]$. Como $\pi_{F}\left[D_{F}\right]$ é denso em $\pi_{F}[Y]$, existe $\pi_{F}(z) \in \pi_{F}\left[D_{F}\right]$ tal que $\pi_{F}(z) \in$ $\pi_{F}[Y] \cap \prod_{i \in F} B_{i}$, ou seja

$$
z \in D_{F} \text { e } z \in \pi_{F}^{-1}\left(\prod_{i \in F} B_{i}\right)=\prod_{i<\omega} B_{i} .
$$

Logo existe $z \in D_{F} \subset D$ tal que $z \in Y \cap \prod_{i<\omega} B_{i}$. Assim, $D$ é denso em $Y$. Concluímos que $X$ é hereditariamente separável.

Definição 3.6. Um espaço topológico $X$ é hereditariamente Lindelöf se todo subespaço $Y \subset X$ é Lindelöf.

Assim, como consequência da Proposicao 3.5, se $X$ é espaço de Banach que é hereditariamente Lindelöf na topologia fraca então, $X$ não possui sistema biortogonal não enumerável.

Teorema 3.8 (Zenor). Se $X^{\omega}$ é hereditariamente separável, então $C(X)$ é hereditariamente Lindelöf na topologia da convergência pontual. 
Demonstração. Consideremos a função $\Psi: X^{\omega} \times C(X) \rightarrow \mathbb{R}^{\omega}$ definida por

$$
\Psi\left(\left(x_{n}\right)_{n}, f\right)=\left(f\left(x_{n}\right)\right)_{n \in \omega} .
$$

Para cada aberto básico $B$ de $\mathbb{R}^{\omega}$ e cada $x \in X^{\omega}$, definamos

$$
N(x, B):=\{f \in C(X): \Psi(x, f) \in B\} .
$$

Afirmação 1: Temos que $\left\{N(x, B): x \in X^{\omega}\right.$ e $B$ é aberto básico de $\left.\mathbb{R}^{\omega}\right\}$ é uma base para a topologia da convergência pontual em $C(X)$.

Demonstração da Afirmação 1. De fato, seja $A$ um aberto básico de $C(X)$ na topologia da convergência pontual.

Lembremos que dado $A$ um aberto básico de $C(X)$ na topologia da convergência pontual, $A$ é da forma $C(X) \cap \prod_{x \in X} A_{x}$ e existem $x_{1}, \ldots, x_{j} \in X$ tais que $A_{x}=\mathbb{R}$ se $x \notin\left\{x_{1}, \ldots, x_{j}\right\}$ e cada $A_{x_{n}}$ é um aberto de $\mathbb{R}$, para $1 \leq n \leq j$.

Suponhamos então que $A=C(X) \cap \prod_{x \in X} A_{x}$, com $A_{x}=\mathbb{R}$ se $x \in X \backslash\left\{x_{1}, \ldots, x_{j}\right\}$. Definamos $y=\left(y_{n}\right)_{n} \in X^{\omega}$ por

$$
y_{n}=\left\{\begin{array}{cll}
x_{n}, & \text { se } 1 \leq n \leq j \\
0, & \text { se } n>j
\end{array}\right.
$$

e $B:=\prod_{n \in \omega} B_{n}$ um aberto básico de $\mathbb{R}^{\omega}$ onde

$$
B_{n}=\left\{\begin{array}{cll}
A_{x_{n}}, & \text { se } & 1 \leq n \leq j \\
\mathbb{R}, & \text { se } & n>j
\end{array}\right.
$$

Se $f \in N(y, B)$, então $\Psi(y, f)=\left(f\left(y_{n}\right)\right)_{n \in \omega} \in B$. Em particular, para $1 \leq n \leq j$, temos que $f\left(y_{n}\right)=f\left(x_{n}\right) \in B_{x_{n}}$. Logo $f \in C(X) \cap \prod_{x \in X} A_{x}$. Assim $N(y, B) \subset C(X) \cap \prod_{x \in X} A_{x}$.

Por outro lado, se $f \in C(X) \cap \prod_{x \in X} A_{x} \subset \prod_{x \in X} A_{x}$, então $f\left(x_{n}\right) \in A_{x_{n}}$ para todo $1 \leq n \leq$ $j$. Logo $\Psi(y, f)=\left(f\left(y_{n}\right)\right)_{n} \in B$ e, portanto, $f \in N(y, B)$. Concluímos assim que $\prod_{x \in X} A_{x} \subset$ $N(y, B) \subset C(X)$.

Logo, $\left\{N(x, B): x \in X^{\omega}\right.$ e $B$ é aberto básico de $\left.\mathbb{R}^{\omega}\right\}$ é base para $C(X)$.

Escolhendo-se $\mathfrak{I}$ uma base enumerável de $\mathbb{R}^{\omega}$ formada de abertos básicos, temos ainda que

$$
\mathfrak{P}=\left\{N(x, B): x \in X^{\omega} \text { e } B \in \mathfrak{I}\right\}
$$

é base para topologia pontual de $C(X)$.

Afirmação 2: Para todo $\mathcal{C} \subset \mathfrak{P}$, existe $\mathcal{D} \subset \mathcal{C}$ enumerável tal que

$$
\bigcup \mathcal{C}=\bigcup \mathcal{D}
$$

Demonstração da Afirmação 2. Seja $\mathcal{C} \subset \mathfrak{P}$.

Para cada $B \in \mathfrak{I}$ seja $Y_{B}=\left\{x \in X^{\omega}: N(x, B) \in \mathcal{C}\right\} \subset X^{\omega}$.

Por hipótese, $X^{\omega}$ é hereditariamente separável, logo existe $D_{B} \subset Y_{B}$ denso enumerável em $Y_{B}$. Definamos então

$$
\mathcal{D}:=\left\{N(x, B): x \in D_{B} \wedge B \in \mathfrak{I}\right\} .
$$

Temos que $\mathcal{D}$ é enumerável e $\bigcup \mathcal{D} \subset \bigcup \mathcal{C}$.

Seja $f \in N(x, B) \in \mathcal{C}$. Suponhamos que $B=\prod_{n<\omega} B_{n} \operatorname{com} B_{n}=\mathbb{R}$ se $n \in \omega \backslash\left\{n_{1}, \ldots, n_{j}\right\}$. Como $f$ é contínua, seja

$$
A_{n_{k}}:=f^{-1}\left[B_{n_{k}}\right] \text { para } 1 \leq k \leq j
$$


aberto em $X$ e

$$
A:=\prod_{n<\omega} A_{n}
$$

com $A_{n}=X$ se $n \notin\left\{n_{1}, \ldots, n_{j}\right\}$.

Temos que $x \in Y_{B}$, pois $N(x, B) \in C$. Por outro lado, como $f \in N(x, B)$, segue-se que $\left(f\left(x_{n}\right)\right)_{n} \in B$. Em particular, $f\left(x_{n_{k}}\right) \in B_{n_{k}}$ para $1 \leq k \leq j$, ou seja, $x_{n_{k}} \in A_{n_{k}}$ para $1 \leq k \leq j$. Assim, $x \in A$. Concluímos portanto que $x \in Y_{B} \cap A$. Logo $Y_{B} \cap A$ é aberto não vazio de $Y_{B}$.

Pela escolha de $D_{B}$, existe $y \in D_{B}$ tal que $y \in Y_{B} \cap A$, e isto implica que $\left(f\left(y_{n}\right)\right)_{n} \in B$ e portanto $f \in N(y, B)$. Como $N(y, B) \in \mathcal{D}$, segue-se que $f \in \bigcup \mathcal{D}$. Assim $\bigcup \mathcal{C}=\bigcup \mathcal{D}$.

Vamos mostrar agora que $C(X)$ é hereditariamente Lindelöf na topologia da convergência pontual.

Seja $Y \subset C(X)$ e $\mathcal{C}$ uma cobertura por abertos de $Y$. Como $\mathfrak{P}$ é base para $C(X)$, podemos supor que $\mathcal{C} \subset \mathfrak{P}$.

Pela Afirmação 2, existe $\mathcal{D} \subset \mathcal{C}$ enumerável tal que $\bigcup \mathcal{D}=\bigcup \mathcal{C}$. Assim $\mathcal{D}$ é uma subcobertura enumerável de $\mathcal{C}$ para o subespaço $Y$.

Concluímos portanto que $C(K)$ é hereditariamente Lindelöf na topologia da convergência pontual.

Recordemos que um espaço topológico $X$ é disperso se todo subespaço de $X$ possui um ponto isolado.

Teorema 3.9. Se K é um compacto Hausdorff disperso, então a topologia fraca em $C(K)$ coincide com a topologia da convergência pontual em $C(K)$.

Demonstração. Ver [21], Teorema 7.4, página 1128.

Concluímos a seção com o teorema que nos da condições suficientes que um espaço compacto $K$ deve satisfazer, de tal modo o espaço de Banach $C(K)$ admita apenas sistemas biortogonais enumeráveis.

Teorema 3.10. Seja $K$ um compacto Hausdorff disperso tal que $K^{n}$ é hereditariamente separável para todo $n \in \omega$. Então $C(K)$ não possui sistema biortogonal não enumerável.

Demonstração. Como $K^{n}$ é hereditariamente separável para todo $n \in \mathbb{N}$, segue-se pelo Lema 3.2 que $K^{\omega}$ é hereditariamente separável. Pelo Teorema 3.8 segue-se que $C(K)$ é hereditariamente Lindelöf na topologia da convergência pontual. Agora como $K$ é compacto Hausdorff disperso, pelo Teorema 3.9 segue-se que a topologia fraca em $C(K)$ coincide com a topologia da convergência pontual em $C(K)$. Concluímos assim que $(C(K), \omega)$ é hereditariamente Lindelöf. Logo, pela Proposição 3.5, $C(K)$ não possui sistemas biortogonais não enumeráveis.

Do Teorema 3.10, se $K$ é compacto disperso não metrizável e tal que $K^{n}$ é hereditariamente separável para todo $n \in \omega$, então $C(K)$ é um espaço de Banach não separável que não possui sistemas biortogonais não enumerável. A construção de um compacto $K$ satisfazendo as propriedades acima será estudada no Capítulo 5. Os espaços compactos satisfazendo as propriedades do Teorema 3.10 são denominados de compactos de Kunen. O primeiro espaço de Kunen foi obtido por K. Kunen, sob a Hipótese do Contínuo².

\footnotetext{
${ }^{2}$ Ver [21], Teorema 7.1, página 1123.
} 


\subsection{Sistemas semibiortogonais}

Nesta seção, vamos estudar uma aplicação de sistemas biortogonais em espaços de Banach. $\mathrm{Na}$ verdade, consideramos uma versão mais fraca de sistemas biortogonais, os chamados sistemas semibiortogonais.

Iniciamos a seção com a definição de conjunto suporte em um espaço de Banach e estudamos algumas propriedades relacionadas a conjuntos suportes. Em particular, estudamos o Teorema 3.11, que diz que se um espaço de Banach $X$ possui um conjunto suporte, então $X$ não pode ser um espaço separável.

Em seguida, definimos os sistemas semi-biortogonais e estudamos o Teorema 3.12. O Principal resultado do Teorema 3.12 é o fato de que para um espaço de Banach, a existência de um conjunto suporte é equivalente a existência de um sistema semibiortogonal.

Concluímos a seção estudando o Teorema 3.13, que nos garante a existência de um sistema biortogonal não enumerável para um espaço de Banach $C(K)$, desde de que $K$ satisfaça certas condições.

Definição 3.7. Seja $C$ um fechado convexo de um espaço de Banach $X$.

- Um ponto $x_{0} \in X$ é um ponto suporte de $C$ se, existe um funcional $x_{0}^{*} \in X^{*}$ tal que

$$
x_{0}^{*}\left(x_{0}\right)=\inf _{x \in C} x_{0}^{*}(x)<\sup _{x \in C} x_{0}^{*}(x) .
$$

- $C$ é um conjunto suporte se todo elemento de $C$ é um ponto suporte de $C$.

Teorema 3.11. Se $X$ é um espaço de Banach separável, então $X$ não possui um conjunto suporte.

Demonstração. Suponha por absurdo que $X$ admita um conjunto suporte $C$. Seja $\left\{x_{n}: n \in \omega\right\}$ um conjunto denso e enumerável em $C$. Tomemos uma sequência $\left(b_{n}\right)_{n}$ de números reais tal que $b_{n}>0$ para todo $n \in \omega, \sum b_{n}=1$ e a série $\sum b_{n} x_{n}$ converge.

Seja $\bar{x}=\sum b_{n} c_{n}$. Então $\bar{x} \in C$ e por hipótese, existe $x_{0}^{*} \in X^{*}$ tal que

$$
x_{0}^{*}(\bar{x})=\inf _{x \in C} x_{0}^{*}(x)<\sup _{x \in C} x_{0}^{*}(x) .
$$

Em particular,

$$
\forall n \in \omega \quad\left(x_{0}^{*}(\bar{x}) \leq x_{0}^{*}\left(x_{n}\right)\right) .
$$

$\operatorname{Mas} x_{0}^{*}(\bar{x})=x_{0}^{*}\left(\sum b_{n} x_{n}\right)=\sum b_{n} x_{0}^{*}\left(x_{n}\right)$. Logo

$$
\forall n \in \omega \quad\left(x_{0}^{*}\left(x_{n}\right)=x_{0}^{*}\left(x_{0}\right)\right) .
$$

Portanto, $x_{0}^{*}$ é constante em $C$, uma contradição com 3.5.1.

Com o Teorema 3.11, podemos nos perguntar se é verdade que um espaço de Banach é separável se, e somente se, possui um conjunto suporte. Porém essa questão é independente de ZFC. A construção de um espaço de Banach não separável sem conjuntos suportes pode ser encontrada em $[15]$.

Vamos estudar agora uma caracterização de conjuntos suportes por outros tipos de estruturas.

Definição 3.8. Seja $X$ um espaço de Banach (real). Um subconjunto $C$ de $X$ é denominado um cone se:

- $C$ é não trivial, ou seja, $C \neq \emptyset$ e $C \neq X$,

- $\lambda C \subset C$ para todo real $\lambda$ não negativo,

- $C$ é convexo, 
- $C$ é fechado,

- $C \cap(-C)=\{0\}$, onde $-C:=\{-c, c \in C\}$.

Lema 3.3. Se $X$ tem um conjunto suporte não vazio, então $X \oplus \mathbb{R}$ tem um cone que é um conjunto suporte.

Demonstração. Seja $C$ um conjunto suporte em $X$. Definamos

$$
S:=\{t(x, 1): t \geq 0, x \in C\}
$$

É fácil ver que $S$ é cone em $X \oplus \mathbb{R}$.

Vamos mostrar que $K$ é um conjunto suporte. Seja $t_{0}\left(x_{0}, 1\right) \in S$.

- Se $t_{0}=0$, tomemos $T: X \oplus \mathbb{R} \rightarrow \mathbb{R}$ dado por $T(x, \lambda)=\lambda$. Então $T \in(X \oplus \mathbb{R})^{*} \mathrm{e}$

$$
T\left(t_{0}\left(x_{0}, 1\right)\right)=T(0,0)=0=\inf _{s \in S} T(s)<1=\sup _{s \in S} T(s) .
$$

- Suponhamos agora que $t_{0} \neq 0$. Seja $x_{0}^{*} \in X^{*}$ tal que

$$
x_{0}^{*}\left(x_{0}\right)=\inf _{x \in C} x_{0}^{*}(x)<\sup _{x \in C} x_{0}^{*}(x) .
$$

Seja $T: X \oplus \mathbb{R} \rightarrow \mathbb{R}$ dada por $T(x, \lambda)=x_{0}^{*}(x)-x_{0}^{*}\left(x_{0}\right) \lambda$. Então $T \in(X \oplus \mathbb{R})^{*}$. Temos que $T(s) \geq 0$ para $s \in S$ e

$$
T\left(t_{0}\left(x_{0}, 1\right)\right)=t_{0}\left(x_{0}^{*}\left(x_{0}\right)-x_{0}^{*}\left(x_{0}\right) 1\right)=0=\inf _{s \in S} T(s) .
$$

Por outro lado.

$$
\sup _{s \in S} T(s)=\sup _{t \geq 0, x \in C} T(t(x, 1)) \geq \sup _{x \in C} T(x, 1)=\sup _{x \in C}\left(x_{0}^{*}(x)-x_{0}^{*}\left(x_{0}\right)\right)>0 .
$$

Logo, $S$ é conjunto suporte.

Lema 3.4. Se $X$ admite um conjunto suporte, então todo subespaço de codimensão finita em $X$ admite um conjunto suporte.

Demonstração. Seja $Y$ um subespaço de codimensão finita de $X$.

Suponhamos primeiramente que $Y$ tem codimensão 1 . Assim existe $\Lambda \in X^{*}$ tal que $Y=\Lambda^{-1}(0)$. Consideremos $C$ um conjunto suporte em $X$.

- Suponhamos que $C \subset \Lambda^{-1}(\alpha)$ para algum $\alpha \in \mathbb{R}$. Fixemos um elemento $c \in C$ e definamos $C^{\prime}:=C-c$. Observemos que $C^{\prime} \subset Y$ e é fácil ver que $C^{\prime}$ é convexo fechado.

Dado $c^{\prime} \in C^{\prime}$, existe $x_{0} \in C$ tal que $c^{\prime}=x_{0}-c$. Seja $x_{0}^{*} \in X^{*}$ tal que

$$
x_{0}^{*}\left(x_{0}\right)=\inf _{x \in C} x_{0}^{*}(x)<\sup _{x \in C} x_{0}^{*}(x) .
$$

Consideremos $y_{0} \in Y^{*}$ a restrição do funcional $x_{0}^{*}$ no subespaço $Y$. Temos então que

$$
\begin{gathered}
y_{0}^{*}\left(c^{\prime}\right)=y_{0}^{*}\left(x_{0}-c\right)=y_{0}^{*}\left(x_{0}\right)-y_{0}^{*}(c)=x_{0}^{*}\left(x_{0}\right)-x_{0}^{*}(c)=\inf _{x \in C}\left(x_{0}^{*}(x)-x_{0}^{*}(c)\right)= \\
=\inf _{y \in C^{\prime}} x_{0}^{*}(y)=\inf _{y \in C^{\prime}} y_{0}^{*}(y) .
\end{gathered}
$$

Por outro lado

$$
\sup _{y \in C^{\prime}} y_{0}^{*}(y)=\sup _{x \in C} x_{0}^{*}(x-c)=\sup _{x \in C}\left(x_{0}^{*}(x)-x_{0}^{*}(c)\right)>x_{0}^{*}\left(x_{0}\right)-x_{0}^{*}(c)=
$$




$$
=x_{0}^{*}\left(x_{0}-c\right)=x_{0}^{*}\left(c^{\prime}\right)=y_{0}^{*}\left(c^{\prime}\right) .
$$

Temos portanto que

$$
y_{0}^{*}\left(c^{\prime}\right)=\inf _{y \in C^{\prime}} y_{0}^{*}(y)<\sup _{y \in C^{\prime}} y_{0}^{*}(y) .
$$

Concluímos assim que $C^{\prime}$ é um conjunto suporte em $Y$.

- Suponhamos agora que $\forall \alpha \in \mathbb{R} \quad\left(C \not \subset \Lambda^{-1}(\alpha)\right)$.

Tomemos $\alpha \in \mathbb{R}$ tal que

$$
\inf _{x \in C} \Lambda(x)<\alpha<\sup _{x \in C} \Lambda(x) .
$$

e seja $C_{\alpha}:=C \cap \Lambda^{-1}(\alpha)$. Temos que $C_{\alpha}$ é fechado convexo e não vazio. $C_{\alpha}$ é não vazio, pois $C$ é convexo e existe $c_{1}, c_{2} \in C$ tal que $\Lambda\left(c_{1}\right)<\alpha<\Lambda\left(c_{2}\right)$.

Vamos mostrar que $C_{\alpha}$ é um conjunto suporte em $\Lambda^{-1}(\alpha)$. Tomemos $x_{0} \in C_{\alpha}$. Como $x_{0} \in C$ e $C$ é um conjunto suporte, existe $\phi \in X^{*}$ tal que

$$
\phi\left(x_{0}\right)=\inf _{x \in C} \phi(x)<\sup _{x \in C} \phi(x) .
$$

Consideremos $\varphi$ a restrição de $\phi$ ao subespaço $\Lambda^{-1}(\alpha)$. Para mostrarmos que $x_{0}$ é um ponto suporte para $C_{\alpha}$, basta encontrarmos $z \in C_{\alpha}$ tal que

$$
\phi(z)>\phi\left(x_{0}\right)
$$

pois neste caso teremos

$$
\inf _{x \in C_{\alpha}} \varphi(x) \leq \inf _{x \in C} \phi(x)=\phi\left(x_{0}\right)<\phi(z)=\varphi(z) \leq \sup _{x \in C_{\alpha}} \varphi(x) .
$$

Fixemos $\overline{x_{0}} \in C$ tal que $\phi\left(\overline{x_{0}}\right)>\phi\left(x_{0}\right)$.

- Se $\overline{x_{0}} \in C_{\alpha}$, basta tomarmos $z=\overline{x_{0}}$ em 3.5.2.

- Suponhamos então que $\overline{x_{0}} \notin C_{\alpha}$, ou seja, $\Lambda\left(\overline{x_{0}}\right) \neq \alpha$. Sem perda de generalidade, suponhamos que $\Lambda\left(\overline{x_{0}}\right)>\alpha$. Escolhemos $y \in C$ tal que $\Lambda(y)<\alpha$. Como $C$ é convexo, existe $t \in(0,1)$ tal que $y+t \overline{x_{0}} \in C$ e $\Lambda\left(y+t \overline{x_{0}}\right)=\alpha$. Assim $y+t \overline{x_{0}} \in C_{\alpha}$ e $\phi\left(y+t \overline{x_{0}}\right)=\phi(y)+t \phi\left(\overline{x_{0}}\right)>\phi\left(x_{0}\right)$. Portanto, basta tomarmos $z:=y+t \overline{x_{0}}$ em 3.5.2.

Assim, $C_{\alpha}$ é um conjunto suporte em $\Lambda^{-1}(\alpha)$.

Fixemos agora $x \in C_{\alpha}$ e definamos $C_{\alpha}^{\prime}:=C_{\alpha}-x$. Temos assim que $C_{\alpha}^{\prime}$ é um conjunto suporte em $Y=\Lambda^{-1}(0)$.

Vamos provar agora o caso geral por indução em $n$, onde $n$ é a codimensão de $Y$.

$\mathrm{O}$ caso $n=1$ já foi provado acima. Suponhamos o resultado válido para todo subespaço de codimensão $n-1$. Assim, $X=Y \oplus\left\langle x_{1}, \ldots, x_{n}\right\rangle=Y \oplus\left\langle x_{n}\right\rangle \oplus\left\langle x_{1}, \ldots, x_{n-1}\right\rangle$.

Logo $Y^{\prime}:=Y \oplus\left\langle x_{n}\right\rangle$ é subespaço de $X$ de codimensão $n-1$. Pela hipótese de indução, existe $C^{\prime} \subset Y^{\prime}$ um conjunto suporte. Agora $Y$ é hiperplano de $Y^{\prime}$, e pelo caso anterior, $Y$ possui um conjunto suporte.

Definição 3.9. Seja $X$ um espaço de Banach e $\kappa$ um cardinal. Um sistema $\left(x_{\alpha}, f_{\alpha}\right)_{\alpha \in \kappa}$ em $X \times X^{*}$ é um sistema semibiortogonal se:

- $f_{\beta}\left(x_{\alpha}\right)=0$ se $\alpha<\beta$,

- $f_{\beta}\left(x_{\beta}\right)=1$ para todo $\beta<\kappa e$

- $f_{\beta}\left(x_{\alpha}\right) \geq 0$ para todo $\alpha, \beta<\kappa$. 
Com os lemas acimas, podemos agora estabelecer o seguinte teorema:

Teorema 3.12. Para um espaço de Banach $X$, as seguintes afirmações são equivalentes:

(i) Existe um sistema semibiortogonal não enumerável $\left(x_{\alpha}, f_{\alpha}\right)_{\alpha<\omega_{1}}$ em $X \times X^{*}$.

(ii) $X$ possui um cone suporte.

(iii) $X$ possui um conjunto suporte limitado (não vazio).

Demonstração. $(i) \rightarrow($ iii $)$. Normalizemos o sistema de forma que $\forall \alpha<\omega_{1}\left(\left\|x_{\alpha}\right\|=1\right)$.

Definamos $C:=\overline{\operatorname{conv}}\left\{x_{\alpha}: \alpha<\omega_{1}\right\}$. Vamos mostrar que $C$ é um conjunto suporte limitado.

Tomemos $y \in C$. Então existe $\left(y_{n}\right)_{n} \subset \operatorname{conv}\left\{x_{\alpha}: \alpha<\omega_{1}\right\}$ que converge para $y$. Como $\omega_{1}$ é regular, existe $\beta<\omega_{1}$ tal que $\left(y_{n}\right)_{n} \subset \operatorname{span}\left\{x_{\alpha}: \alpha<\beta\right\}$ e, portanto, $y \in \overline{\operatorname{span}}\left\{x_{\alpha}: \alpha<\beta\right\}$. Logo

$$
0=f_{\beta+1}(y)=\inf _{x \in C} f_{\beta+1}(x)<1=f_{\beta+1}\left(x_{\beta+1}\right) \leq \sup _{x \in C} f_{\beta+1}(x) .
$$

Assim, $C$ é um conjunto suporte. Além disso, para todo $x \in C$ temos que $\|x\|=1$.

$($ iii $) \rightarrow($ ii $)$. Sejam $x \in X$ e $Y$ um subespaço de $X$ tal que $X=Y \oplus\langle x\rangle$. Por hipótese, $X$ tem um conjunto suporte e, como $Y$ é subespaço de codimensão finita de $X$, segue-se pelo Lema 3.4 que $Y$ tem um conjunto suporte. Agora, como $Y$ tem conjunto suporte, $Y \oplus\langle x\rangle \equiv Y \oplus \mathbb{R}$ tem cone suporte pelo Lema 3.3. Logo, $X$ tem cone suporte.

$(i i) \rightarrow(i)$. Seja $S \subset X$ um cone suporte. Vamos construir um sistema semibiortogonal $\left(x_{\alpha}, f_{\alpha}\right)_{\alpha<\omega_{1}}$ em $X \times X^{*}$.

Fixemos $y \in S$ e seja (pela propriedade de $S$ ser suporte ) $f_{0} \in X^{*}$ tal que

$$
f_{0}(y)=\inf _{x \in S} f_{0}(x)<\sup _{x \in S} f_{0}(x) .
$$

Como $S$ é cone, para todo $t \geq 0$ temos que $t S \subset S$. Logo, devemos ter que $f_{0}(y)=0$ (caso contrário, se $f_{0}\left(x_{0}\right)=\lambda>0$, então $\frac{y}{2} \in S$ e $f_{0}\left(\frac{y}{2}\right)=\frac{\lambda}{2}<\lambda=\inf _{x \in S} f_{0}(x)$ ). Em particular, para todo $x \in S$, temos que $f_{0}(x) \geq 0$.

Seja agora $x_{0} \in S_{X} \cap S$ tal que $f_{0}\left(x_{0}\right)=1$ (caso não exista, basta multiplicarmos $f_{0}$ por uma constante positiva).

Seja $\mu<\omega_{1}$ e suponhamos que temos um sistema $\left(x_{\alpha}, f_{\alpha}\right)_{\alpha<\mu}$ em $\left(S_{X} \cap S\right) \times X^{*}$ tal que

1. $\forall \alpha<\mu \quad\left(f_{\alpha}\left(x_{\alpha}\right)=1\right)$,

2. $\forall \beta<\alpha<\mu \quad\left(f_{\alpha}\left(x_{\beta}\right)=0\right)$,

3. $\forall \alpha, \beta \in \mu \quad\left(f_{\alpha}\left(x_{\beta}\right) \geq 0\right)$.

Seja $\bar{x}=\sum_{\alpha<\mu} c_{\alpha} x_{\alpha}$, onde $\sum_{\alpha<\mu} c_{\alpha}=1$ e $\forall \alpha<\mu\left(c_{\alpha}>0\right)$.

Então, $\bar{x} \in S$. Seja $f_{\mu} \in X^{*}$ tal que

$$
f_{\mu}(\bar{x})=\inf _{x \in S} f_{\mu}(x)<\sup _{x \in S} f_{\mu}(x) .
$$

Novamente, $f_{\mu}(\bar{x})=0$ pois $K$ é cone. Assim,

$$
0=f_{\mu}(\bar{x})=f_{\mu}\left(\sum_{\alpha<\mu} c_{\alpha} x_{\alpha}\right)=\sum_{\alpha<\mu} c_{\alpha} f_{\mu}\left(x_{\alpha}\right) .
$$

Além disso, como $f_{\mu}(\bar{x})=\inf _{x \in S} f_{\mu}(x)$, segue-se que $0 \leq f_{\mu}(x)$ para todo $x \in S$. Logo $\forall \alpha<$ $\mu\left(f_{\mu}\left(x_{\alpha}\right)=0\right)$. Seja então $x_{\mu} \in S_{X} \cap K$ tal que $f_{\mu}\left(x_{\mu}\right)=1$.

Observe que $\left(x_{\alpha}, f_{\alpha}\right)_{\alpha<\omega_{1}}$ construído desta forma é um sistema semibiortogonal.

A consistência de que todo espaço de Banach não separável possui um conjunto suporte é consequência da consistência de que todo espaço de Banach não separável possui um sistema biortogonal não enumerável e do Teorema 3.12, pois todo sistema biortogonal é claramente um sistema semi-biortogonal. 
Estudaremos agora a existência de conjuntos suportes para espaços de Banach da forma $C(K)$. Primeiramente, temos o seguinte resultado:

Proposição 3.6. Se $K$ é um espaço compacto Hausdorff e $F$ é um fechado não $G_{\delta}$ de $K$, então

$$
C:=\{f \in C(K): f[F]=\{0\}, f \geq 0\}
$$

é um cone suporte e $C \cap\{f:\|f\| \leq r\}$ é um conjunto suporte limitado em $C(K)$ para todo $r>0$.

Demonstração. Da definição segue que $C$ é cone. Vejamos que é um conjunto suporte.

Seja $f \in C$. Temos que $f^{-1}(0)$ é um $G_{\delta}$ e, portanto, $F$ é um subconjunto próprio de $f^{-1}(0)$. Seja então $x \in K$ tal que $x \notin F$ e $f(x)=0$. Consideremos o funcional $\delta_{x} \in C(K)^{*}$, definido por $\delta_{x}(g)=g(x)$ para todo $g \in C(K)$. Então

$$
\delta_{x}(f)=f(x)=0=\inf _{g \in C} \delta_{x}(g) .
$$

Observemos que a ultima igualdade segue-se do fato de que $g \geq 0$ para todo $g \in C$.

Por outro lado, como $x \notin F$, existe $h: K \rightarrow[0,1]$ tal que $h[F] \subset\{0\}$ e $h(x)=1$. Assim ${ }^{3}$

$$
1=h(x)=\delta_{x}(h) \leq \sup _{g \in C} \delta_{x}(g) .
$$

Concluímos portanto que

$$
\delta_{x}(f)=f(x)=0=\inf _{g \in C} \delta_{x}(g)<\sup _{g \in C} \delta_{x}(g) .
$$

Logo $f$ é um ponto suporte de $C$. Como $f \in C$ era arbitrário, concluímos que $C$ é um conjunto suporte.

Seja agora $r>0$ e consideremos $f \in C_{r}:=C \cap\{f:\|f\| \leq r\}$. Pela demonstração da primeira parte, existe $T \in C(K)^{*}$ tal que

$$
0=T(f)=\inf _{g \in C} T(g)<\sup _{g \in C} T(g) .
$$

Seja $h \in C$ tal que $T(h)>0$. Assim $\frac{r}{\|h\|} h \in C_{r}$ e

$$
0=T(f)=\inf _{g \in C_{r}} T(g)<T\left(\frac{r}{\|h\|} h\right) \leq \sup _{g \in C_{r}} T(g) .
$$

Concluímos assim que $C_{r}$ é um conjunto suporte.

Pela Proposição 3.6 , se $K$ possui um fechado que não é um $G_{\delta}$, então o espaço de Banach $C(K)$ possui um conjunto suporte, e pelo Teorema 3.12, isto implica que $C(K)$ possui um sistema semi-biortogonal não enumerável. Assim podemos restringir o estudo da existência de sistemas semibiortogonais não enumeráveis para espaços de Banach da forma $C(K)$, nos quais todo fechado de $K$ são $G_{\delta}$.

Neste caso, veremos que uma condição suficiente para a existência de sistemas semi-biortogonais é a de que o espaço compacto $K$ possua peso maior que $\omega_{1}$, e este é o conteúdo do Teorema 3.13. Na verdade, obteremos neste caso a existência de um sistema biortogonal não enumerável. Para a demonstração do Teorema 3.13, precisamos antes de um lema.

Definição 3.10. Seja $K$ um compacto Hausdoff. Para $f \in C(K)$ e $r \in \mathbb{R}$ definamos

- $\left.\left.K(f \leq r):=f^{-1}(] \infty, r\right]\right)$.

- $K(f<r):=f^{-1}(] \infty, r[)$.

\footnotetext{
${ }^{3}$ Observemos que $h \in C$.
} 
- $K(f \geq r)=K \backslash K(f<r)$.

- $K(f>r)=K \backslash K(f \leq r)$.

Lema 3.5. Seja $K$ compacto Hausdorff de peso maior que $\omega_{1}$. Então existe uma família crescente e contínua $\left(F_{\gamma}\right)_{\gamma \in \omega_{2}}$ de subconjuntos de $C(K)$ de tamanho no máximo $\omega_{1}$ tal que cada $F_{\gamma}$ é fechado pelas seguintes operações:

1) $f \in F_{\gamma}$ implica $-f \in F_{\gamma}$.

2) Se $K(f \leq p) \cap K(g \geq q)=\emptyset$ para $f, g \in F_{\gamma}$ e $p, q \in \mathbb{Q}$, então existe $h \in F_{\gamma}$ tal que

$$
h_{\mid K(f \leq p)} \equiv 0 \text { e } h_{\mid K(g \geq q)} \equiv 1 .
$$

Mais ainda, para cada $\gamma \in \omega_{1}$, existe $x_{\gamma}, y_{\gamma} \in K$ e $f_{\gamma}: K \rightarrow[0,1]$ em $F_{\gamma+1}$ tal que:

3) $f_{\gamma}\left(x_{\gamma}\right)=0$ e $f_{\gamma}\left(y_{\gamma}\right)=1$.

4) $\forall g \in F_{\gamma}\left(g\left(x_{\gamma}\right)=g\left(y_{\gamma}\right)\right)$.

Se todo fechado de $K$ é $G_{\delta}$, então podemos no lugar de 3$)$ ter

$\left.3^{\prime}\right) f_{\gamma}^{-1}(0)=\left\{x_{\gamma}\right\}$ e $f_{\gamma}^{-1}(0)=\left\{y_{\gamma}\right\}$.

Demonstração. Começamos a demonstração com a seguinte afirmação:

Afirmação $S e F \subset C(K)$ e $|F| \leq \omega_{1}$, então $F$ não separa pontos de $K$.

Demonstração da Afirmação. De fato, suponhamos que $F$ separa pontos de $K$. Seja $A$ um aberto não vazio de $K$ e tomemos $x \in A$. Para todo $y \in X \backslash A$, existe por hipótese $g \in F$ e $q_{y} \in \mathbb{Q}$ tal que

$$
x \in C_{y}:=g^{-1}(]-\infty, q[) \text { e } y \in B_{y}:=g^{-1}(] q, \infty[),
$$

$\mathrm{Ou}$

$$
y \in B_{y}:=g^{-1}(]-\infty, q[) \text { e } x \in C_{y}:=g^{-1}(] q, \infty[) .
$$

Assim, $K \backslash A \subset \bigcup_{y \in K} B_{y}$. Por compacidade, existem $y_{1}, \ldots, y_{n} \in K$ tal que $K \backslash A \subset \bigcup_{i=1}^{n} B_{y_{i}}$. Logo, $x \in \bigcap_{i=1}^{n} C_{y_{i}} \subset A$.

Mostramos assim que

$$
B:=\left\{g^{-1}(]-\infty, q[): g \in F, q \in \mathbb{Q}\right\} \cup\left\{g^{-1}(] q, \infty[): g \in F, q \in \mathbb{Q}\right\}
$$

é uma sub-base para $K$. Observe que $|B| \leq \omega_{1}$, contradizendo o fato de que $K$ tem peso maior que $\omega_{1}$, o que conclui a demonstração da Afirmação.

Vamos agora construir a sequência $\left(F_{\gamma}\right)_{\gamma<\omega_{2}}$ por indução.

Seja $F_{0}:=\emptyset$.

Se $\gamma<\omega_{2}$ é limite, definamos $F_{\gamma}:=\bigcup_{\delta<\gamma} F_{\delta}$.

Para qualquer $\gamma<\omega_{2}$, suponhamos que temos definido $\left(F_{\alpha}\right)_{\alpha \leq \gamma}$ satisfazendo as condições 1) a 4) e vamos construir $F_{\gamma+1}$ fazendo uma indução sobre os naturais.

Pelo Afirmação, como $\left|F_{\gamma}\right| \leq \omega_{1}$, existem $x_{\gamma}, y_{\gamma} \in K$ tais que

$$
\forall g \in F_{\gamma} \quad\left(g\left(x_{\gamma}\right)=g\left(y_{\gamma}\right)\right) .
$$

Seja então $f_{\gamma} \in C(K)$ tal que $f_{\gamma}\left(x_{\gamma}\right)=0$ e $f_{\gamma}\left(y_{\gamma}\right)=1$ (no caso de $\{x\}$ e $\{y\}$ serem $G_{\delta}$, podemos tomar $f_{\gamma}$ satisfazendo $f_{\gamma}^{-1}(0)=\left\{x_{\gamma}\right\}$ e $f_{\gamma}^{-1}(0)=\left\{y_{\gamma}\right\}$ ). Definamos $F_{\gamma+1}^{0}:=F_{\gamma} \cup\left\{f_{\gamma},-f_{\gamma}\right\}$.

Suponhamos definido $F_{\gamma+1}^{n}$ e definamos

$$
Q:=\left\{\langle\langle p, q\rangle,\langle f, g\rangle\rangle \in \mathbb{Q}^{2} \times\left(F_{\gamma+1}^{n}\right)^{2}: K(f \leq p) \cap K(g \geq q)=\emptyset\right\} .
$$

Para cada elemento $z=\langle\langle p, q\rangle,\langle f, g\rangle\rangle \in Q$, escolhemos um elemento $h^{z} \in C(K)$ tal que

$$
h_{\mid K(f \leq p)}^{z} \equiv 0 \text { e } h_{\mid K(g \geq q)}^{z} \equiv 1 .
$$


Definamos então

$$
F_{\gamma+1}^{n+1}:=F_{\gamma+1}^{n} \cup\left\{h^{z}: z \in Q\right\} \cup\left\{-h^{z}: z \in Q\right\} .
$$

Por construção, para cada $n \in \omega$ vale que se $f \in F_{\gamma+1}^{n}$ então $-f \in F_{\gamma+1}^{n}$ e se $f, g \in F_{\gamma+1}^{n}$ satisfazem $K(f \leq p) \cap K(g \geq q)=\emptyset$, então existe $h \in F_{\gamma+1}^{n+1}$ tal que

$$
h_{\mid K(f \leq p)} \equiv 0 \text { e } h_{\mid K(g \geq q)} \equiv 1 \text {. }
$$

Deste modo, definindo

$$
F_{\gamma+1}:=\bigcup_{n \in \omega} F_{\gamma+1}^{n},
$$

temos que a família $\left(F_{\alpha}\right)_{\alpha<\gamma+2}$ satisfaz as propriedades de 1) a 4 ).

Por indução transfinita, obtemos a família $\left(F_{\alpha}\right)_{\alpha<\omega_{2}}$ como no enunciado.

Teorema 3.13 (S. Todorcevic [25]). Seja K um compacto Hausdorff de peso maior que $\omega_{1}$. Se todo fechado de $K$ é um $G_{\delta}$, então $C(K)$ admite um sistema biortogonal não enumerável.

Demonstração. Sejam $\left(F_{\gamma}\right)_{\gamma<\omega_{2}}$ uma família de subconjuntos de $C(K)$ e pontos $\left(x_{\gamma}, y_{\gamma}\right)_{\gamma<\omega_{2}}$ como no enunciado do Lema 3.5.

Para cada $\gamma<\omega_{2}$, tomemos

$$
U_{\gamma}:=\left\{K(f<p), K(g>q): f, g \in F_{\gamma}, p, q \in \mathbb{Q}\right\} .
$$

Observemos que, pela continuidade da família $\left(F_{\gamma}\right)_{\gamma<\omega_{2}}$, segue-se que para todo ordinal limite $\gamma<\omega_{2}$

$$
U_{\gamma}:=\bigcup_{\alpha<\gamma} U_{\alpha}
$$

Assim, a sequência $\left(U_{\alpha}\right)_{\alpha<\omega_{2}}$ é contínua. Além disso, ela é crescente.

Fixemos agora $\gamma<\omega_{2}$ de cofinalidade $\omega_{1}$.

Afirmação 1. Existe $\xi_{\gamma}<\gamma$ tal que para todos $p, q \in \mathbb{Q}$

$$
\begin{gathered}
\bigcup\left\{V \in U_{\gamma}: V \subset K\left(f_{\gamma}<p\right)\right\}=\bigcup\left\{V \in U_{\xi_{\gamma}}: V \subset K\left(f_{\gamma}<p\right)\right\} \\
e \\
\bigcup\left\{V \in U_{\gamma}: V \subset K\left(f_{\gamma}>q\right)\right\}=\bigcup\left\{V \in U_{\xi_{\gamma}}: V \subset K\left(f_{\gamma}>q\right)\right\} .
\end{gathered}
$$

Demonstração da Afirmação 1. Temos que $\gamma$ é ordinal limite. Temos então que, para $p \in \mathbb{Q}$,

$$
\bigcup\left\{V \in U_{\gamma}: V \subset K\left(f_{\gamma}<p\right)\right\}=\bigcup_{\alpha<\gamma} \bigcup\left\{V \in U_{\alpha}: V \subset K\left(f_{\gamma}<p\right)\right\} .
$$

Para cada $\alpha<\gamma$, definamos

$$
B_{\alpha}:=\bigcup\left\{V \in U_{\alpha}: V \subset K\left(f_{\gamma}<p\right)\right\} .
$$

Por hipótese, todo aberto de $K$ é $F_{\sigma}, \operatorname{logo}$ existem $F_{n}, n \in \omega$, fechados tais que

$$
\bigcup_{n \in \omega} F_{n}=\bigcup\left\{V \in U_{\gamma}: V \subset K\left(f_{\gamma}<p\right)\right\}=\bigcup_{\alpha<\gamma} B_{\alpha} .
$$

Assim, para todo $n \in \omega \quad\left(F_{n} \subset \bigcup_{\alpha<\gamma} B_{\alpha}\right)$. 
Fixemos $n_{0} \in \omega . F_{n_{0}}$ é fechado em $K$, logo é compacto. Assim, existe $y \subset \gamma$ finito tal que

$$
F_{n_{0}} \subset \bigcup_{\alpha \in y} B_{\alpha}=B_{\sup y}
$$

Daí, para todo $n \in \omega$ existe $\alpha_{n} \in \gamma$ tal que $F_{n} \subset B_{\alpha_{n}}$. Como $c f(\gamma)=\omega_{1}$, existe $\beta_{p} \in \gamma$ tal que para todo $n\left(\alpha_{n}<\beta_{p}\right)$.

Logo para todo $n \in \omega \quad\left(F_{n} \subset B_{\alpha_{n}} \subset B_{\beta_{p}}\right)$.

Assim, $\bigcup F_{n} \subset B_{\beta_{p}}$. Logo,

$$
\bigcup\left\{V \in U_{\gamma}: V \subset K\left(f_{\gamma}<p\right)\right\}=B_{\beta_{p}}=\bigcup\left\{V \in U_{\beta_{p}}: V \subset K\left(f_{\gamma}<p\right)\right\} .
$$

Temos portanto uma aplicação $\beta: \mathbb{Q} \rightarrow \gamma$ dada por $\beta(p)=\beta_{p}$.

Como $c f(\gamma)=\omega_{1}$, existe $\phi<\gamma$ tal que $\forall p \in \mathbb{Q}\left(\beta_{p}<\phi\right)$. Assim, para todo $p \in \mathbb{Q}$,

$$
\bigcup\left\{V \in U_{\gamma}: V \subset K\left(f_{\gamma}<p\right)\right\}=\bigcup\left\{V \in U_{\phi}: V \subset K\left(f_{\gamma}<p\right)\right\} .
$$

De modo análogo, podemos encontrar $\phi^{\prime}<\gamma$ tal que para todo $p \in \mathbb{Q}$.

$$
\bigcup\left\{V \in U_{\gamma}: V \subset K\left(f_{\gamma}>p\right)\right\}=\bigcup\left\{V \in U_{\phi}: V \subset K\left(f_{\gamma}>p\right)\right\} .
$$

Seja $\xi_{\gamma}=\max \left\{\phi, \phi^{\prime}\right\}$. Então $\xi<\gamma$ e para todos $p, q \in \mathbb{Q}$,

$$
\begin{gathered}
\bigcup\left\{V \in U_{\gamma}: V \subset K\left(f_{\gamma}<p\right)\right\}=\bigcup\left\{V \in U_{\xi_{\gamma}}: V \subset K\left(f_{\gamma}<p\right)\right\} \\
\text { e } \\
\bigcup\left\{V \in U_{\gamma}: V \subset K\left(f_{\gamma}>q\right)\right\}=\bigcup\left\{V \in U_{\xi_{\gamma}}: V \subset K\left(f_{\gamma}>q\right)\right\},
\end{gathered}
$$

como queríamos.

Seja então $S:=\left\{\gamma<\omega_{2}: c f(\gamma)=\omega_{1}\right\}$. Temos que $S$ é estacionário em $\omega_{2}$ e pela Afirmação 1 , temos uma aplicação regressiva $f: S \rightarrow \omega_{2}$, onde $f(\gamma)=\xi_{\gamma}$.

Pelo Teorema de Fodor 2.11, existe $\Gamma \subset S$ estacionário e $\beta<\omega$ tal que $f_{\mid \Gamma} \equiv \beta$. Assim, para todo $\gamma \in \Gamma$ e para todos $p, q \in \mathbb{Q}$,

$$
\begin{gathered}
\bigcup\left\{V \in U_{\gamma}: V \subset K\left(f_{\gamma}<p\right)\right\}=\bigcup\left\{V \in U_{\beta}: V \subset K\left(f_{\gamma}<p\right)\right\} \\
\text { e } \\
\bigcup\left\{V \in U_{\gamma}: V \subset K\left(f_{\gamma}>q\right)\right\}=\bigcup\left\{V \in U_{\beta}: V \subset K\left(f_{\gamma}>q\right)\right\} .
\end{gathered}
$$

Afirmação 2. $f_{\delta}\left(x_{\gamma}\right)=f_{\delta}\left(y_{\gamma}\right)$ para todos $\delta, \gamma \in \Gamma \operatorname{com} \gamma<\delta$.

Demonstração da Afirmação 2. Suponhamos por absurdo que existam $\delta, \gamma \in \Gamma \operatorname{com} \gamma<\delta$ e tais que $f_{\delta}\left(x_{\gamma}\right) \neq f_{\delta}\left(y_{\gamma}\right)$. Então existem racionais $p<q$ tais que

1) $f_{\delta}\left(x_{\gamma}\right)<p<q<f_{\delta}\left(y_{\gamma}\right)$ ou

2) $f_{\delta}\left(y_{\gamma}\right)<p<q<f_{\delta}\left(x_{\gamma}\right)$.

Suponhamos que ocorre 1).

Temos que

$$
K \backslash K\left(f_{\delta}<p\right)=K\left(f_{\delta} \geq p\right) \subset \bigcup_{q>0} K\left(f_{\gamma}>q\right),
$$

pois $f_{\gamma}^{-1}(\{0\})=\left\{x_{\gamma}\right\}$ e $x_{\gamma} \notin K\left(f_{\delta} \geq p\right)$ ( e lembrando que $f_{\gamma}: K \rightarrow[0,1]$ ). 
Como $K$ é compacto, existem $0<q_{0}, \ldots, q_{n}$ racionais tais que

$$
K\left(f_{\delta} \geq p\right) \subset \bigcup_{i=0}^{n} K\left(f_{\gamma}>q_{i}\right)=K\left(f_{\gamma}>q_{0}\right) .
$$

Assim, $x_{\gamma} \in K\left(f_{\gamma} \leq q_{0}\right) \subset K\left(f_{\delta}<p\right)$. Como $0<q_{0}$ e $f_{\gamma}\left(x_{\gamma}\right)=0$, podemos tomar $0<r<q_{0}$ e assim obtemos que

$$
x_{\gamma} \in K\left(f_{\gamma}<r\right) \subset K\left(f_{\delta}<p\right) .
$$

De modo análogo, existe $s \in \mathbb{Q}$ tal que

$$
y_{\gamma} \in K\left(f_{\gamma}>s\right) \subset K\left(f_{\delta}>q\right) .
$$

Pela definição de $U_{\delta}$ e lembrando que $F_{\delta}=\bigcup_{\xi<\delta} F_{\xi}$, temos que $K\left(f_{\gamma}<r\right), K\left(f_{\gamma}>s\right) \in U_{\delta}$.

Pela escolha de $\beta$ segue que

$$
\bigcup\left\{V \in U_{\delta}: V \subset K\left(f_{\delta}<p\right)\right\}=\bigcup\left\{V \in U_{\delta}: V \subset K\left(f_{\delta}<p\right)\right\} .
$$

Como $x_{\gamma} \in K\left(f_{\delta}<r\right) \in U_{\delta}$, segue-se que $x_{\gamma} \in \bigcup\left\{V \in U_{\delta}: V \subset K\left(f_{\delta}<p\right)\right\}$. Logo, deve existir $V \in U_{\beta}$ tal que

$$
x_{\gamma} \in V \subset K\left(f_{\delta}<p\right) .
$$

De modo análogo, deve existir $W \in U_{\beta}$ tal que

$$
y_{\gamma} \in W \subset K\left(f_{\delta}>q\right) .
$$

Como $V, W \in U_{\beta}$, devem existir $g, h \in F_{\beta}$ e $t, v \in \mathbb{Q}$ tais que:

1) $V=K(g<t), W=K(h>v)$

2) $V=K(g>t), W=K(h<v)$

3) $V=K(g<t), W=K(h<v)=K(-h>-v)$ (análogo ao Caso 1)

4) $V=K(g>t), W=K(h>v)=K(-h<-v)$ (análogo ao Caso 2).

Suponha que ocorre 1).

Assim,

$$
\begin{gathered}
x_{\gamma} \in K(g<t) \subset K\left(f_{\delta}<p\right) \mathrm{e} \\
y_{\gamma} \in K(h>v) \subset K\left(f_{\delta}>q\right) .
\end{gathered}
$$

Observemos que podemos tomar $t, v \in \mathbb{Q}$ tal que $K(g \leq t) \cap K(h \geq v)=\emptyset^{4}$.

Assim

$$
K(g \leq t) \cap K(h \geq v) \subset K\left(f_{\delta}<p\right) \cap K\left(f_{\delta}>q\right)=\emptyset .
$$

Pela Propriedade 2) do Lema 3.5, existe $f \in F_{\beta}$ tal que

$$
f_{\mid K(g \leq t)} \equiv 0 \text { e } f_{\mid K(h \geq v)} \equiv 1 .
$$

Em particular,

$$
f\left(x_{\gamma}\right)=0 \neq 1=f\left(y_{\gamma}\right) .
$$

Porém, $\beta<\min \Gamma \leq \gamma$ e, pela Propriedade 4) do Lema 3.5, $g^{\prime}\left(x_{\gamma}\right)=g^{\prime}\left(y_{\gamma}\right)$ para todo $g^{\prime} \in F_{\sigma}$ com $\sigma<\gamma$, uma contradição com 3.5.4.

Com isto, concluímos a demonstração da Afirmação 2).

Para cada $\gamma \in \Gamma$, seja $\phi_{\gamma}=\delta_{y_{\gamma}}-\delta_{x_{\gamma}}$.

Então, $\left(f_{\gamma}, \phi_{\gamma}\right)_{\gamma \in \Gamma}$ é um sistema biortogonal não enumerável em $C(K)$.

\footnotetext{
${ }^{4}$ De fato, $g\left(x_{\gamma}\right)<t_{1}<t$ para um certo $t_{1} \in \mathbb{Q}$ e $h\left(y_{\gamma}\right)>v_{1}>v$ para um certo $v_{1} \in \mathbb{Q}$, logo basta tomarmos $t=t_{1}$ e $v=v_{1}$
} 
Teorema 3.14. Todo espaço de Banach $C(K)$ de densidade maior que $\omega_{1}$ possui um sistema semi-biortogonal não enumerável.

Demonstração. Se $K$ possui um fechado que não é $G_{\delta}$, o resultado segue da Proposição 3.6 e do Teorema 3.12 .

Se todo fechado de $K$ é um $G_{\delta}$, então o resultado segue do Teorema 3.13 . 


\section{Capítulo 4}

\section{Funções cardinais em termos de sistemas biortogonais}

Neste capítulo, estudamos algumas funções cardinais em espaços de Banach associados aos sistemas biortogonais. Generalizamos um resultado de S.Todorcevic [24] para espaços compactos Hausdorff quaisquer e obtivemos um resultado para espaços compactos Hausdorff análogo ao Teorema de McKenzie para álgebras de Boole.

Iniciamos o capítulo com a Seção 4.1, onde apresentamos as funções cardinais que utilizaremos nas próximas seções e estudamos algumas propriedades básicas dessas funções. Na Seção 4.2, estudamos a relação entre os sistemas biortogonais em $C(K)$ e o peso topológico de $K$, para $K$ um compacto Hausdorff. Em particular, obtemos nessa seção o Teorema 4.2, que é uma generalização do Teorema 4.1 de S. Todorcevic [24] para compactos Hausdorff quaisquer. Na Seção 4.3 estudamos a noção de irredundância para algebras de Boole, espaços de Banach e álgebras de Banach. Definimos uma nova função cardinal associada à irredundância para álgebras de Banach $C(K) \mathrm{e}$ obtemos o Teorema 4.6, que é uma versão do Teorema 4.5 de McKenzie.

\subsection{Funções cardinais de espaços topológicos e de Banach}

Nesta seção, estudaremos algumas funções cardinais que serão utilizadas nas próximas seções.

Funções cardinais associam a cada elemento de uma certa classe de espaços (topológicos, espaços de Banach, etc) um cardinal que tem por objetivo mensurar certa propriedade.

Começamos introduzindo algumas funções cardinais:

Definição 4.1. Seja X um espaço topológico.

- O peso topológico de $X$ é definido por

$$
w(X):=\min \{|\mathfrak{B}|: \mathfrak{B} \text { é uma base para } X\} .
$$

- A densidade de $X$ é definida por

$$
d(X):=\min \{|D|: D \text { é um subconjunto denso de } X\} .
$$

- O grau de Lindelöf de $X$ é definido por

$$
h L(X):=\sup \{L(Y): Y \subset X\},
$$

onde

$L(Y):=\min \{\kappa:$ toda cobertura por abertos de $Y$ possui uma subcobertura de cardinalidade $\kappa\}$. 
- $O$ caráter de $X$ é definido por

$$
\chi(X):=\sup \{\chi(p, X): p \in X\}
$$

onde dado $p \in X, \chi(p, X):=\min \{|V|: V$ é base para $p\}$.

As funções acima definidas e suas principais propriedades encontram-se em R.Hodel, Cardinal functions[11].

Definição 4.2. Seja X um espaço de Banach.

- $\operatorname{biort}(X):=\sup \{\kappa$ : existe um sistema biortogonal de cardinalidade $\kappa\}$.

- $\operatorname{sbiort}(X):=\sup \{\kappa:$ existe um sistema semibiortogonal de cardinalidade $\kappa\}$.

Para espaços compactos, temos ainda a seguinte definição:

Definição 4.3. Seja K um compacto Hausdorff.

- Um sistema biortogonal nice em $C(K) \times C(K)^{*}$ de cardinalidade $\kappa$ é um sistema biortogonal da forma $\left(f_{\alpha}, \delta_{x_{\alpha}}-\delta_{y_{\alpha}}\right)_{\alpha<\kappa}$ em $C(K) \times C(K)^{*}$.

- Definamos o cardinal nbiort $2(K)$ por

$$
\text { nbiort }_{2}(K):=\sup \{\kappa: \text { existe um sistema biortogonal nice de cardinalidade } \kappa\} \text {. }
$$

Mencionamos aqui o artigo [16] de P.Koszmider, para as funções cardinais associados aos sistemas biortogonais.

Uma grande parte dos estudos relacionados a sistemas biortogonais está ligado aos cardinais $d(X)$ e biort $(X)$ para um espaço de Banach $X$.

Nas próximas seções, relacionaremos as funções cardinais relacionadas a sistemas biortogonais em espaços de Banach $C(K)$ com as funções cardinais definidas para o compacto $K$. Com este intuito, estudaremos nesta seção o comportamento de algumas funções cardinais para espaços compactos Hausdorff.

Começamos com o seguinte lema:

Lema 4.1. Seja K um compacto Hausdorff. Então:

$$
h L(K)=\varphi(K),
$$

onde $\varphi(K):=\min \{\kappa:$ todo fechado de $K$ é intersecção de $\kappa$ abertos $\}$.

Demonstração. Pela definição de $h L(K)$, é fácil ver que $h L(K)=\sup \{L(B): B$ aberto em $K\}$ e, como complemento de aberto é um fechado, também temos que $\varphi(K)=\min \{\kappa:$ todo aberto de $K$ é a união de $\kappa$ fechados $\}$.

Vamos primeiramente mostrar que $h L(K) \leq \varphi(K)$.

Seja $B$ um aberto de $K$ e $\mathcal{B}:=\left(B_{\alpha}\right)_{\alpha \in \Gamma}$ um cobertura por abertos de $B$. Por hipótese, existem $\left(F_{\alpha}\right)_{\alpha<\varphi(K)}$ fechados tais que

$$
B=\bigcup_{\alpha \in \varphi(K)} F_{\alpha}=\bigcup_{\alpha \in \Gamma} B_{\alpha} .
$$

Para cada $\beta \in \varphi(K)$, temos que $F_{\beta}$ é compacto e $F_{\beta} \subset \bigcup_{\alpha \in \Gamma} B_{\alpha}$. Logo existe um conjunto finito $\Gamma_{\beta} \subset \Gamma$ tal que $F_{\beta} \subset \bigcup_{\alpha \in \Gamma_{\beta}} B_{\alpha}$. Assim

$$
B=\bigcup\left\{B_{\alpha}: \alpha \in \Gamma_{\beta}, \beta \in \varphi(K)\right\} .
$$

Achamos portanto um subconjunto de $\mathcal{B}$ de cardinalidade no máximo $\varphi(K)$ que ainda cobre $B$. Como $B$ era arbitrário, concluímos que $h L(K) \leq \varphi(K)$. 
Mostraremos agora que $\varphi(K) \leq h L(K)$.

Seja $B$ um aberto de $K$. Para cada $x \in B$, existe pela regularidade do espaço $K$, uma vizinhança aberta $V_{x}$ de $x$ tal que $x \in V_{x} \in \overline{V_{x}} \subset B$. Temos então que $B=\bigcup_{x \in B} V_{x}$. Por hipótese, existe $E \subset B$ com $|E| \leq h L(K)$ tal que $B=\bigcup_{x \in E} V_{x}$. Logo $B=\bigcup_{x \in E} \overline{V_{x}}$. Mostramos portanto que todo aberto de $K$ pode ser escrito como a união de menos que $h L(K)$ fechados. Assim, $\varphi(K) \leq h L(K)$.

Lema 4.2. Seja K compacto Hausdorff. Então:

$$
\chi(K) \leq h L(K) .
$$

Demonstração. Seja $x \in K$. Pelo Lema 4.1, existem abertos $\left(B_{\alpha}\right)_{\alpha \in h L(K)}$ tais que $\{x\}=\bigcap\left\{B_{\alpha}\right.$ : $\alpha \in h L(K)\}$. Como $K$ é regular, para cada $\alpha \in h L(K)$, seja $A_{\alpha}$ um aberto de $K$ tal que $x \in A_{\alpha} \subset$ $\overline{A_{\alpha}} \subset B_{\alpha}$.

Afirmação: $\left\{A_{\alpha}: \alpha \in h L(K)\right\}$ é uma subbase para o ponto $x$.

Demonstração. Seja $G$ um aberto que contém $x$. Então $\{x\}=\bigcap\left\{\overline{A_{\alpha}}: \alpha \in h L(K)\right\} \subset G, \operatorname{logo}$ $K \backslash G \subset \bigcup\left\{K \backslash \overline{A_{\alpha}}: \alpha \in h L(K)\right\}$. Como $K \backslash G$ é compacto, existe $\Gamma \subset h L(K)$ finito tal que $K \backslash G \subset \bigcup\left\{K \backslash \overline{A_{\alpha}}: \alpha \in \Gamma\right\}$. Logo,

$$
x \in \bigcap_{\alpha \in \Gamma} A_{\alpha} \subset \bigcap_{\alpha \in \Gamma} \overline{A_{\alpha}} \subset G .
$$

Assim, $\left\{A_{\alpha}: \alpha \in h L(K)\right\}$ é sub-base para $x$.

Concluímos da Afirmação que $\chi(K) \leq h L(K)$.

Lema 4.3. Seja $X$ um espaço topológico normal. Dado $x \in X$, existe uma família $F_{x}$ de funções contínuas, de cardinalidade no máximo $\chi(X)$, tal que $\left\{f^{-1}(]-\infty, 1[): f \in F_{x}\right\}$ forma uma base para $x$.

Demonstração. Seja $x \in X$ e $\mathcal{V}_{x}$ uma base para $x$ de cardinalidade menor ou igual a $\chi(X)$.

Para cada $A \in \mathcal{V}_{x}$, seja pelo Lema de Urysohn, $f_{A}: X \rightarrow[0,1]$ tal que $f_{A}(x)=0$ e $f_{A}(y)=1$ se $y \in X \backslash A$. Então $F_{x}:=\left\{f_{A}: A \in V_{x}\right\}$ satisfaz as condições do lema.

\subsection{Sistemas biortogonais e peso topológico}

Obteremos nesta seção, para todo espaço compacto Hausdorff $K$, uma relação entre as funções cardinais $w(K), h L(K)$ e nbiort $t_{2}(K)$. Essa relação é uma modificação do Teorema 3.13.

Para espaços booleanos, temos o seguinte resultado devido a S.Todorcevic:

Teorema 4.1 (S. Todorcevic [24]). Para K um espaço booleano, temos que

$$
w(K) \leq \text { nbiort }_{2}(K) \cdot h L(K)^{+} .
$$

Demonstração. Ver [24], Proposição 1.

Com uma modificação da demonstração do Teorema 3.13, obteremos uma generalização da Desigualdade 4.2.1 para todo espaço compacto Hausdorff, não necessariamente booleano.

Começamos provando uma modificação do Lema 3.5. Antes recordemos a seguinte definição:

Definição 4.4. Seja $K$ um compacto Hausdoff. Para $f \in C(K)$ e $r \in \mathbb{R}$ definamos

- $\left.\left.K(f \leq r):=f^{-1}(] \infty, r\right]\right)$,

- $K(f<r):=f^{-1}(] \infty, r[)$,

- $K(f \geq r)=K \backslash K(f<r)$, 
- $K(f>r)=K \backslash K(f \leq r)$.

Lema 4.4. Seja $K$ compacto Hausdorff de peso $\kappa$ e tal que $h L(K)^{+}<\kappa$. Então existe uma família crescente e contínua $\overline{F_{\gamma}}(\gamma \in \kappa)$ de subconjuntos de $C(K)$ de tamanho estritamente menor que $\kappa$ tal que cada $F_{\gamma}$ é fechado pelas seguintes operações:

1) $f \in F_{\gamma}$ implica $-f \in F_{\gamma}$.

2) Se $K(f \leq p) \cap K(g \geq q)=\emptyset$ para $f, g \in F_{\gamma}$ e $p, q \in \mathbb{Q}$, então existe $h \in F_{\gamma}$ tal que

$$
h_{\mid K(f \leq p)} \equiv 0 \text { e } h_{\mid K(g \geq q)} \equiv 1 .
$$

Além disso, a família $F_{\gamma}(\gamma \in \kappa)$ satisfaz:

Para cada $\gamma \in \kappa$, existe $x_{\gamma}, y_{\gamma} \in K$ e $f_{\gamma}: K \rightarrow[0,1]$ em $F_{\gamma+1}$ tal que:

3) $f_{\gamma}\left(x_{\gamma}\right)=0$ e $f_{\gamma}\left(y_{\gamma}\right)=1$.

4) Para todo $g \in F_{\gamma}$, temos que $g\left(x_{\gamma}\right)=g\left(y_{\gamma}\right)$.

E para cada $\alpha \in \kappa$

5) Se $x_{\alpha} \in V$ com $V$ aberto, então existe $f \in F_{\alpha+1}$ tal que

$$
x_{\alpha} \in K(f<1) \subset V,
$$

6) Se $y_{\alpha} \in V$ com $V$ aberto, então existe $g \in F_{\alpha+1}$ tal que

$$
y_{\alpha} \in K(g>0) \subset V \text {. }
$$

Demonstração. Seja $K$ compacto Hausdorff de peso $\kappa$ e tal que $h L(K)^{+}<\kappa$.

Afirmação : Se $F \subset C(K)$ e $|F|<\kappa$, então $F$ não separa pontos de $K$.

Demonstração da Afirmação. De fato, suponhamos que $F$ separa pontos de $K$. Seja $A$ um aberto não vazio de $K$.

Tomemos $x \in A$. Para todo $y \in K \backslash A$ existe por hipótese $g \in F$ e $q \in \mathbb{Q}$ tal que

$$
x \in C_{y}:=g^{-1}(]-\infty, q[) \text { e } y \in B_{y}:=g^{-1}(] q, \infty[)
$$

$\mathrm{Ou}$

$$
y \in B_{y}:=g^{-1}(]-\infty, q[) \text { e } x \in C_{y}:=g^{-1}(] q, \infty[) .
$$

Assim $K \backslash A \subset \bigcup_{y \in K} B_{y}$. Por compacidade, existem $y_{1}, \ldots, y_{n} \in K$ tal que $K \backslash A \subset \bigcup_{i=1}^{n} B_{y_{i}}$. Logo $x \in \bigcap_{i=1}^{n} C_{y_{i}} \subset A$.

Mostramos assim que

$$
\mathfrak{B}:=\left\{g^{-1}(]-\infty, q[): g \in F, q \in \mathbb{Q}\right\} \cup\left\{g^{-1}(] q, \infty[): g \in F, q \in \mathbb{Q}\right\}
$$

é uma sub-base para $K$, contradizendo o fato que $K$ tem peso $\kappa$ e $|\mathfrak{B}| \leq|F|<\kappa$.

Vamos construir a sequência $F_{\alpha}(\alpha<\kappa)$ por indução.

- Seja $F_{0}:=\emptyset$.

Dado $\beta<\kappa$, suponhamos que temos construídos $F_{\alpha}(\alpha \in \beta)$ satisfazendo as propriedades 1) e 2) tal que

$$
\forall \alpha<\beta \quad\left(\left|F_{\alpha}\right| \leq \omega \cdot h L(K) \cdot|\alpha|\right)
$$

e temos construídos $x_{\alpha}, y_{\alpha}$ para $\alpha<\beta$ satisfazendo as condições de 3) a 6).

- Caso $\beta$ limite: Definamos $F_{\beta}:=\bigcup_{\delta<\beta} F_{\delta}$.

Então $F_{\beta}$ satisfaz as condições 1) e 2) e $\left|F_{\beta}\right| \leq \omega \cdot h L(K) \cdot|\beta|$. 
- Caso $\beta=\gamma+1$ :

Pela Afirmação, como $\left|F_{\gamma}\right| \leq \omega \cdot h L(K) \cdot|\alpha|<\kappa$, existem $x_{\gamma}, y_{\gamma} \in K$ tais que

$$
\forall g \in F_{\gamma}\left(g\left(x_{\gamma}\right)=g\left(y_{\gamma}\right)\right) .
$$

Seja então $f_{\gamma} \in C(K)$ tal que $f_{\gamma}\left(x_{\gamma}\right)=0$ e $f_{\gamma}\left(y_{\gamma}\right)=1$. Consideremos as famílias de funções $F_{x_{\gamma}} \subset C(K)$ e $F_{y_{\gamma}} \subset C(K)$ dadas pelo Lema 4.3. Vamos construir $F_{\gamma+1}$ fazendo uma indução sobre os naturais.

Definamos $F_{\gamma+1}^{0}:=F_{\gamma} \cup\left\{f_{\gamma},-f_{\gamma}\right\} \cup\left\{f,-f: f \in F_{x_{\gamma}}\right\} \cup\left\{g,-g: g \in F_{y_{\gamma}}\right\}$. Suponhamos definido $F_{\gamma+1}^{n}$. Definamos

$$
Q:=\left\{\langle\langle p, q\rangle,\langle f, g\rangle\rangle \in \mathbb{Q}^{2} \times\left(F_{\gamma+1}^{n}\right)^{2}: K(f \leq p) \cap K(g \geq q)=\emptyset\right\} .
$$

Para cada elemento $z=\langle\langle p, q\rangle,\langle f, g\rangle\rangle \in Q$ escolhemos um elemento $h^{z} \in C(K)$ tal que

$$
h_{\mid K(f \leq p)}^{z} \equiv 0 \text { e } h_{\mid K(g \geq q)}^{z} \equiv 1 .
$$

Definamos então

$$
F_{\gamma+1}^{n+1}:=F_{\gamma+1}^{n} \cup\left\{h^{z}: z \in Q\right\} \cup\left\{-h^{z}: z \in Q\right\}
$$

Por fim, seja

$$
F_{\gamma+1}:=\bigcup_{n \in \omega} F_{\gamma+1}^{n}
$$

Observemos que, para cada $n \in \omega \quad\left(\left|F_{\gamma+1}^{n}\right| \leq\left|F_{\gamma}\right| \cdot h l(K) \leq \omega \cdot h L(K) \cdot|\gamma+1|\right)$.

Teorema 4.2. Seja K um compacto Hausdorff. Então:

$$
w(K) \leq \text { nbiort }_{2}(K) \cdot h L(K)^{+} .
$$

Demonstração. Com o Lema 4.4, a demonstração do teorema é análoga à demonstração do Teorema 3.13 .

Suponhamos que $h L(K)^{+}<w(K)$. Seja $\lambda$ um cardinal regular tal que $h L(K)^{+}<\lambda \leq w(K)$. Consideremos pelo Lema 4.4 uma família $\left(F_{\gamma}\right)_{\gamma<\lambda}$ de subconjuntos de $C(K)$ tal que cada conjunto da família tenha tamanho estritamente menor que $\kappa$ e $x_{\alpha}, y_{\alpha} \in K$ para $\alpha<\lambda$ satisfazendo:

1) $f \in F_{\gamma}$ implica $-f \in F_{\gamma}$.

2) Se $K(f \leq p) \cap K(g \geq q)=\emptyset$ para $f, g \in F_{\gamma}$ e $p, q \in \mathbb{Q}$, então existe $h \in F_{\gamma}$ tal que

$$
h_{\mid K(f \leq p)} \equiv 0 \text { e } h_{\mid K(g \geq q)} \equiv 1 .
$$

Para cada $\gamma \in \lambda$, existe $x_{\gamma}, y_{\gamma} \in K$ e $f_{\gamma}: K \rightarrow[0,1]$ em $F_{\gamma+1}$ tal que:

3) $f_{\gamma}\left(x_{\gamma}\right)=0$ e $f_{\gamma}\left(y_{\gamma}\right)=1$

4) $\forall g \in F_{\gamma}\left(g\left(x_{\gamma}\right)=g\left(y_{\gamma}\right)\right)$.

E para cada $\alpha \in \lambda$

5) Se $x_{\alpha} \in V$ com $V$ aberto, então existe $f \in F_{\alpha+1}$ tal que

$$
x_{\alpha} \in K(f<1) \subset V .
$$

6) Se $y_{\alpha} \in V$ com $V$ aberto, então existe $g \in F_{\alpha+1}$ tal que

$$
y_{\alpha} \in K(g>0) \subset V .
$$


Definamos para cada $\gamma<\lambda$

$$
U_{\gamma}:=\left\{K(f<p), K(g>q): f, g \in F_{\gamma}, p, q \in \mathbb{Q}\right\} .
$$

Observemos que, pela continuidade da família $\left(F_{\gamma}\right)_{\gamma<\lambda}$, segue-se que, para todo ordinal limite $\gamma<\lambda$,

$$
U_{\gamma}:=\bigcup_{\alpha<\gamma} U_{\alpha}
$$

Assim, a sequência $\left(U_{\alpha}\right)_{\alpha<\lambda}$ é contínua. Além disso, ela é crescente.

Fixemos agora $\gamma<\lambda$ de cofinalidade $h L(K)^{+}$.

Afirmação 1. Existe $\xi_{\gamma}<\gamma$ tal que para todos $p, q \in \mathbb{Q}$

$$
\begin{aligned}
& \bigcup\left\{V \in U_{\gamma}: V \subset K\left(f_{\gamma}<p\right)\right\}=\bigcup\left\{V \in U_{\xi_{\gamma}}: V \subset K\left(f_{\gamma}<p\right)\right\} . \\
& \bigcup\left\{V \in U_{\gamma}: V \subset K\left(f_{\gamma}>q\right)\right\}=\bigcup\left\{V \in U_{\xi_{\gamma}}: V \subset K\left(f_{\gamma}>q\right)\right\} .
\end{aligned}
$$

Demonstração da Afirmação 1. Como $\gamma$ é ordinal limite, podemos escrever para todo $p \in \mathbb{Q}$ :

$$
\bigcup\left\{V \in U_{\gamma}: V \subset K\left(f_{\gamma}<p\right)\right\}=\bigcup_{\alpha<\gamma} \bigcup\left\{V \in U_{\alpha}: V \subset K\left(f_{\gamma}<p\right)\right\}
$$

Definamos para todo $\alpha<\gamma$

$$
B_{\alpha}:=\bigcup\left\{V \in U_{\alpha}: V \subset K\left(f_{\gamma}<p\right)\right\} .
$$

Por definição de $h L(K)$, existe $\Gamma_{p} \subset \gamma$ de cardinalidade menor ou igual a $h L(K)$ tal que

$$
\bigcup\left\{V \in U_{\gamma}: V \subset K\left(f_{\gamma}<p\right)\right\}=\bigcup_{\alpha \in \Gamma_{p}} B_{\alpha} .
$$

Como $\left|\Gamma_{p}\right| \leq h L(K)$ e $\gamma$ tem cofinalidade $h L(K)^{+}$, existe $\beta_{p} \in \gamma$ tal que $\sup \Gamma_{p} \leq \beta_{p}$. Assim:

$$
\bigcup\left\{V \in U_{\gamma}: V \subset K\left(f_{\gamma}<p\right)\right\}=\bigcup_{\alpha \in \Gamma_{p}} B_{\alpha}=B_{\beta_{p}} .
$$

Temos portanto uma aplicação $\beta: \mathbb{Q} \rightarrow \gamma$ dada por $\beta(p)=\beta_{p}$. Como $c f(\gamma)=\omega_{1}$, existe $\phi<\gamma$ tal que para todo $p \in \mathbb{Q}$ temos que $\beta_{p}<\phi$.

Assim, para todo $p \in \mathbb{Q}$,

$$
\bigcup\left\{V \in U_{\gamma}: V \subset K\left(f_{\gamma}<p\right)\right\}=\bigcup\left\{V \in U_{\phi}: V \subset K\left(f_{\gamma}<p\right)\right\} .
$$

De modo análogo, podemos encontrar $\phi^{\prime}<\gamma$ tal que, para todo $p \in \mathbb{Q}$,

$$
\bigcup\left\{V \in U_{\gamma}: V \subset K\left(f_{\gamma}>p\right)\right\}=\bigcup\left\{V \in U_{\phi}: V \subset K\left(f_{\gamma}>p\right)\right\} .
$$

Seja então $\xi_{\gamma}=\max \left\{\phi, \phi^{\prime}\right\}$. Então $\xi_{\gamma}<\gamma$ e para todos $p, q \in \mathbb{Q}$

$$
\begin{aligned}
& \bigcup\left\{V \in U_{\gamma}: V \subset K\left(f_{\gamma}<p\right)\right\}=\bigcup\left\{V \in U_{\xi_{\gamma}}: V \subset K\left(f_{\gamma}<p\right)\right\} \\
& \bigcup\left\{V \in U_{\gamma}: V \subset K\left(f_{\gamma}>q\right)\right\}=\bigcup\left\{V \in U_{\xi_{\gamma}}: V \subset K\left(f_{\gamma}>q\right)\right\} .
\end{aligned}
$$


Consideremos $S:=\left\{\gamma<\lambda: c f(\gamma)=h L(K)^{+}\right\}$. Temos que $S$ é estacionário em $\lambda$ e pela Afirmação 1, temos uma aplicação regressiva $f: S \rightarrow \lambda$, onde $f(\gamma)=\xi_{\gamma}$.

Pelo Teorema de Fodor 2.11, existe $\Gamma \subset S$ estacionário e $\beta<\lambda$ tal que $f_{\mid \Gamma} \equiv \beta$. Assim, para todo $\gamma \in \Gamma$ e para todos $p, q \in \mathbb{Q}$,

$$
\begin{aligned}
& \bigcup\left\{V \in U_{\gamma}: V \subset K\left(f_{\gamma}<p\right)\right\}=\bigcup\left\{V \in U_{\beta}: V \subset K\left(f_{\gamma}<p\right)\right\} \\
& \bigcup\left\{V \in U_{\gamma}: V \subset K\left(f_{\gamma}>q\right)\right\}=\bigcup\left\{V \in U_{\beta}: V \subset K\left(f_{\gamma}>q\right)\right\} .
\end{aligned}
$$

Afirmação 2. $f_{\delta}\left(x_{\gamma}\right)=f_{\delta}\left(y_{\gamma}\right)$ para todos $\delta, \gamma \in \Gamma \operatorname{com} \gamma<\delta$.

Demonstração da Afirmação 2. Suponhamos que existam $\delta, \gamma \in \Gamma \operatorname{com} \gamma<\delta$ e tais que $f_{\delta}\left(x_{\gamma}\right) \neq$ $f_{\delta}\left(y_{\gamma}\right)$. Então existem racionais $p<q$ tais que

I) $f_{\delta}\left(x_{\gamma}\right)<p<q<f_{\delta}\left(y_{\gamma}\right)$ ou

II) $f_{\delta}\left(y_{\gamma}\right)<p<q<f_{\delta}\left(x_{\gamma}\right)$.

Suponhamos que ocorre $I)$.

Temos então que $x_{\gamma} \in K\left(f_{\delta}<p\right)$ e $y_{\gamma} \in K\left(f_{\delta}>q\right)$. Pela construção da família $F_{\alpha}(\alpha<\lambda)$, existem $f, g \in F_{\gamma+1} \subset F_{\delta}$ tais que

$$
x_{\gamma} \in K(f<1) \subset K\left(f_{\delta}<p\right) \text { e } y_{\gamma} \in K(g<1) \subset K\left(f_{\delta}>q\right) .
$$

Pela definição de $U_{\delta}$, temos que $K(f<1), K(g<1) \in U_{\delta}$.

Logo, $x_{\gamma} \in K(f<1) \subset \bigcup\left\{V \in U_{\delta}: V \subset K\left(f_{\delta}<p\right)\right\}$. Pela escolha de $\beta$,

$$
\bigcup\left\{V \in U_{\delta}: V \subset K\left(f_{\delta}<p\right)\right\}=\bigcup\left\{V \in U_{\delta}: V \subset K\left(f_{\delta}<p\right)\right\} .
$$

Logo, deve existir $V \in U_{\beta}$ tal que

$$
x_{\gamma} \in V \subset K\left(f_{\delta}<p\right) .
$$

De modo análogo, deve existir $W \in U_{\beta}$ tal que

$$
y_{\gamma} \in W \subset K\left(f_{\delta}>q\right) .
$$

Como $V, W \in U_{\beta}$, devem existir $g, h \in F_{\beta}$ e $t, v \in \mathbb{Q}$ tais que, um dos seguintes casos ocorra:

1) $V=K(g<t), W=K(h>v)$

2) $V=K(g>t), W=K(h<v)$

3) $V=K(g<t), W=K(h<v)=K(-h>-v)$ (análogo ao Caso 1)

4) $V=K(g>t), W=K(h>v)=K(-h<-v)$ (análogo ao Caso 2).

Suponha que ocorre 1$)$.

Assim,

$$
x_{\gamma} \in K(g<t) \subset K\left(f_{\delta}<p\right) \text { e } y_{\gamma} \in K(h>v) \subset K\left(f_{\delta}>q\right) .
$$

Observemos que podemos tomar $t, v \in \mathbb{Q}$ tais que $x_{\gamma} \in K(g \leq t)$ e $y_{\gamma} \in K(h \geq v)^{1}$.

Assim,

$$
K(g \leq t) \cap K(h \geq v) \subset K\left(f_{\delta}<p\right) \cap K\left(f_{\delta}>q\right)=\emptyset .
$$

Pela propriedade 2) da família $\left(F_{\gamma}\right)_{\gamma<\lambda}$, existe $h \in F_{\beta}$ tal que

$$
f_{\mid K(g \leq t)} \equiv 0 \text { e } f_{\mid K(h \geq v)} \equiv 1 .
$$

Assim, em particular, $f\left(x_{\gamma}\right)=0 \neq 1=f\left(y_{\gamma}\right)$.

Porém, $\beta<\min \Gamma \leq \gamma$ e, por hipótese, $g\left(x_{\gamma}\right)=g\left(y_{\gamma}\right)$ para todo $g \in F_{\sigma} \operatorname{com} \sigma<\gamma$.

Com isto, concluímos a demonstração da Afirmação 2.

\footnotetext{
${ }^{1}$ De fato, $g\left(x_{\gamma}\right)<t_{1}<t$ para um certo $t_{1} \in \mathbb{Q}$ e $h\left(y_{\gamma}\right)>v_{1}>v 4$ para um certo $v_{1} \in \mathbb{Q}$, logo basta tomarmos $t=t_{1}$ e $v=v_{1}$
} 
Para cada $\gamma \in \Gamma$, seja $\phi_{\gamma}=\delta_{y_{\gamma}}-\delta_{x_{\gamma}}$.

Então $\left(f_{\gamma}, \phi_{\gamma}\right)_{\gamma \in \Gamma}$ é um sistema biortogonal nice de tamanho $\lambda$ em $C(K)$.

Logo $\lambda \leq$ biort $_{2}(K)$ para todo cardinal regular $\lambda \leq w(K)$. Assim $w(K) \leq$ biort $_{2}(K)$.

Corolário 4.1. Seja K um compacto Hausdorff. Então

$$
d(C(K)) \leq \operatorname{sbiort}(C(K))^{+} .
$$

Demonstração. Para todo espaço compacto Hausdorff $L$, temos as desigualdades:

$$
\text { nbiort }_{2}(L) \leq \operatorname{biort}(C(L)) \leq \operatorname{sbiort}(C(L)) .
$$

Da Proposição 3.6 e do Lema 4.1, segue-se que

$$
h L(K) \leq \operatorname{sbiort}(C(K)) .
$$

Assim, pelo Teorema 4.2 concluímos que

$$
w(K) \leq \text { nbiort }_{2}(K) \cdot h L(K)^{+} \leq \operatorname{sbiort}(C(K))^{+} .
$$

O Corolário 4.1 é uma resposta afirmativa para a Questão 6.7 de [16] restrita a espaços de Banach da forma $C(K)$.

\subsection{Irredundância e biortogonalidade}

Estudaremos nesta seção a noção de irredundância para álgebras de Boole e para espaços de Banach da forma $C(K)$. Definiremos funções cardinais relacionadas à irredundância e estudaremos suas relações com as funções cardinais associadas a sistemas biortogonais.

Em particular, veremos que a existência de sistemas biortogonais nice para um espaço de Banach $C(K)$ está associada à existência de conjuntos irredundantes em $C(K)$.

Por fim, obteremos um teorema sobre irredundância para espaços de Banach da forma $C(K)$ análogo ao Teorema de McKenzie para álgebras de Boole.

Iniciamos com uma definição geral de irredundância:

Definição 4.5. Seja $\mathfrak{S}$ uma classe de estruturas com uma familia fixa de subestruturas. Sejam $S \in \mathfrak{S}$ e $R \subset S$. Dizemos que $R$ é irredundante se, e somente se, para todo $r \in R$ existe uma subestrutura de $S$ contendo $R \backslash\{r\}$ e não contendo $r$.

$A$ irredundância $\operatorname{irr}_{\mathfrak{S}}(S)$ de $S$ é o supremo das cardinalidades de subconjuntos irredundantes de $S$.

Usaremos as seguintes classes de estruturas:

- $\mathfrak{S}=B A$ - Álgebras de Boole e subálgebras.

- $\mathfrak{S}=B a S$ - Espaços de Banach e subespaços fechados.

- $\mathfrak{S}=B a A$ - Álgebras de Banach da forma $C(K)$ e subálgebras fechadas.

Observemos que, para um compacto Hausdorff $K$, temos os cardinais $\operatorname{irr}_{B a A}(C(K))$ e $\operatorname{irr}_{B a S}(C(K))$. No caso em que $K$ é o espaço de Stone da álgebra $A$, temos ainda o cardinal $\operatorname{irr}_{B A}(A)$. Vamos estudar a relação entre esses cardinais.

Começamos relacionando os cardinais $i r r_{B A}, \operatorname{irr}_{B a A}$ e $i r r_{B a S}$ :

Proposição 4.1. Seja A uma álgebra de Boole e K um compacto.

1. $\operatorname{irr}_{B A}(A) \leq \operatorname{irr}_{B a A}(C(S(A)))$. 
2. $\operatorname{irr}_{B a A}(C(K)) \leq i r r_{B a S}(C(K))$.

Demonstração. $\quad$ 1. Seja $S \subset A$ um conjunto irredundante.

Consideremos

$$
S^{\prime}=\left\{\chi_{s(a)}: a \in S\right\} \subset C(S(A)) .
$$

Vamos mostrar que $S^{\prime}$ é irredundante em $C(S(A))$. Seja $a \in S$. Por hipótese, $a \in A \backslash\langle S \backslash\{a\}\rangle$. Pelo Lema 1.2, existem $p, q \in S(A)$ tais que $\chi_{s(a)}(p) \neq \chi_{s(a)}(q)$, mas para todo $b \in A \backslash\langle S \backslash\{a\}\rangle$ temos que $\chi_{s(b)}(p)=\chi_{s(b)}(q)$. Logo, $\chi_{s(a)} \notin \overline{\left\langle S^{\prime} \backslash\left\{\chi_{s(a)}\right\}\right\rangle}$.

Concluímos assim que $S^{\prime}$ é irredundante em $C(S(A))$.

2. Segue-se do fato que toda subálgebra de Banach fechada é, em particular, um subespaço fechado.

O próximo resultado, relaciona a noção de irredundância para um espaço de Banach com a noção de biortogonalidade.

Proposição 4.2. Seja $X$ um espaço de Banach e $\left(x_{\alpha}\right)_{\alpha \in \kappa}$ uma sequência de pontos de $X$. Existe uma sequência $\left(x_{\alpha}^{*}\right)_{\alpha \in \kappa}$ de pontos de $X^{*}$ tal que $\left(x_{\alpha}, x_{\alpha}^{*}\right)_{\alpha \in \kappa}$ forma um sistema biortogonal se, $e$ somente se, $x_{\alpha} \notin \operatorname{span}\left(\left\{x_{\beta}: \beta \neq \alpha\right\}\right)$ para todo $\alpha \in \kappa$. Em particular,

$$
\operatorname{irr}_{B a S}(X)=\operatorname{biort}(X) .
$$

Demonstração. $(\Rightarrow)$. Suponhamos que exista uma sequência $\left(x_{\alpha}^{*}\right)_{\alpha \in \kappa}$ de pontos de $X^{*}$ tal que $\left(x_{\alpha}, x_{\alpha}^{*}\right)_{\alpha \in \kappa}$ forma um sistema biortogonal $X \times X^{*}$. Então, para todo $\alpha \in \kappa, x_{\alpha} \notin \operatorname{Ker}\left(x_{\alpha}^{*}\right)$. Como $\overline{\operatorname{span}\left(\left\{x_{\beta}: \beta \neq \alpha\right\}\right)} \subset \operatorname{Ker}\left(x_{\alpha}^{*}\right)$, segue-se que $x_{\alpha} \notin \overline{\operatorname{span}\left(\left\{x_{\beta}: \beta \neq \alpha\right\}\right)}$.

$(\Leftarrow)$ Suponhamos agora que $x_{\alpha} \notin \overline{\operatorname{span}\left(\left\{x_{\beta}: \beta \neq \alpha\right\}\right)}$ para todo $\alpha \in \kappa$. Para $\alpha$ fixo, seja $\left.Y:=\overline{\operatorname{span}\left(\left\{x_{\beta}: \beta \neq \alpha\right\}\right)}\right)$. Então $x_{\alpha} \notin Y$. Seja, pelo Teorema de Hahn-Banach, $x_{\alpha}^{*} \in X^{*}$ tal que $x_{\alpha}^{*}\left(x_{\alpha}\right)=1$ e $x_{\alpha}^{*}(y)=0$ para todo $y \in Y$.

Então $\left(x_{\alpha}, x_{\alpha}^{*}\right)_{\alpha \in \kappa}$ é um sistema biortogonal.

Como vimos na Proposição 4.1, todo subconjunto irredundante no sentido de álgebras de Banach em $C(K)$ é em particular irredundante no sentido de espaços de Banach em $C(K)$. O próximo resultado, relaciona um conjunto irredundante no sentido de álgebras de Banach em $C(K)$ com um sistema biortogonal nice em $C(K)$.

Teorema 4.3. Seja $K$ um compacto Hausdorff. $F \subset C(K)$ é irredundante se, e somente se, para todo $f \in F$ existem $x_{f}, y_{f} \in K$ tais que $f\left(x_{f}\right)-f\left(y_{f}\right)>0$ e $f\left(x_{g}\right)=f\left(y_{g}\right)$ para distintos $f, g \in F$. Em particular,

$$
\operatorname{irr}_{B a A}(C(K))=\text { nbiort }_{2}(K) .
$$

Demonstração. $(\Leftarrow)$ Suponha que $F \subset C(K)$ e para cada $f \in F$ existam $x_{f}, y_{f} \in K$ tais que $f\left(x_{f}\right)-f\left(y_{f}\right)>0$ e $f\left(x_{g}\right)=f\left(y_{g}\right)$ para distintos $f, g \in F$.

Então dado $f \in F$, a subálgebra fechada gerada por $F \backslash\{f\}$ não separa os pontos $x_{f}$ e $y_{f}$. Logo, $f$ não pode pertencer a álgebra gerada por $F \backslash\{f\}$.

$(\Rightarrow)$ Suponhamos que $F$ é irredundante. Seja $f \in F$ e $A$ a subálgebra fechada gerada por $F \backslash\{f\}$. Vamos determinar os pontos $x_{f}, y_{f}$.

Definamos em $K$ a relação

$$
x E y \Leftrightarrow \forall g \in A(g(x)=g(y)) .
$$

Seja $L=K / E$ com a topologia quociente e $\varphi: K \rightarrow L$ a aplicação quociente. 
Para cada $g \in A$, definamos $[g]: L \rightarrow \mathbb{R}$ por $[g]([x])=g(x)$. Por definição da relação $E$ sobre $K,[g]$ está bem definida. Além disso, $[g] \circ \varphi=g$ é contínua. Logo, $[g]: L \rightarrow \mathbb{R}$ é contínua, ou seja, $[g] \in C(L)$. Assim, $\{[g]: g \in A\} \subset C(K)$.

Afirmação: $\{[g]: g \in A\}$ é fechado em $C(L)$.

Demonstração. De fato, suponhamos que $\left(\left[g_{n}\right]\right)_{n}$ converge para $f \in C(L)$. Temos que

$$
\begin{gathered}
\left\|\left[g_{n}\right]-f\right\|_{\infty}^{C(L)}=\sup _{x \in L}\left|\left[g_{n}\right](x)-f(x)\right|=\sup _{x \in K}\left|\left[g_{n}\right](\varphi(x))-f(\varphi(x))\right|= \\
=\sup _{x \in K}\left|g_{n}(x)-f \circ \varphi(x)\right|=\left\|g_{n}-f \circ \varphi\right\|_{\infty}^{C(K)} .
\end{gathered}
$$

Logo, a convergência de $\left(\left[g_{n}\right]\right)_{n}$ para $f$ em $C(L)$ implica a convergência de $\left(g_{n}\right)_{n}$ para $f \circ \varphi$ em $C(K)$. Como $A$ é fechado em $C(K)$, temos que $f \circ \varphi \in A$. Mas $[f \circ \varphi]=f$. Logo, $f \in\{[g]: g \in A\}$.

Concluímos portanto que $\{[g]: g \in A\}$ é fechado em $C(L)$.

Pela Afirmação, temos que $\{[g]: g \in A\}$ é fechado. Além disso, separa pontos de $L$ e contém as funções constantes. Logo, pelo Teorema de Stone Weierstrass 1.7, $C(L)=\{[g]: g \in A\}$.

Suponhamos agora que $f$ é constante em cada classe de $L$. Então podemos definir $[f] \in C(L)$. Assim, $[f]=[g]$ para algum $g \in A$ e, portanto, $f=g \in A$, uma contradição. Logo, existem $x, y \in K$ tais que $\varphi(x)=\varphi(y)$ (ou seja para todo $g \in$ temos $A(g(x)=g(y))$ ) e tais que $f(x) \neq f(y)$. Assim, basta definirmos $\left\{x_{f}, y_{f}\right\}=\{x, y\}$ de tal modo que $f\left(x_{f}\right)-f\left(y_{f}\right)>0$.

Vamos mostrar agora a igualdade $\operatorname{irr}_{B a A}(C(K))=$ nbiort $_{2}(K)$.

Seja $F \subset C(K)$ irredundante e suponhamos que $F=\left\{f_{\alpha}: \alpha<\kappa\right\}$, onde $\kappa=|F|$. Então, para cada $\alpha<\kappa$, existem pontos $x_{\alpha}, y_{\alpha} \in K$ tais que $f_{\alpha}\left(x_{\alpha}\right)-f_{\alpha}\left(y_{\alpha}\right)>0$ e $f_{\alpha}\left(x_{\beta}\right)=f_{\alpha}\left(y_{\beta}\right)$ para distintos $\alpha, \beta<\kappa$. Assim, $\left(\frac{f_{\alpha}}{f_{\alpha}\left(x_{\alpha}\right)-f_{\alpha}\left(y_{\alpha}\right)}, \delta_{x_{\alpha}}-\delta_{y_{\alpha}}\right)_{\alpha<\kappa}$ é um sistema biortogonal nice. Logo,

$$
\operatorname{irr}_{B a A}(C(K)) \leq \text { nbiort }_{2}(K) .
$$

Por outro lado, dado $\left(f_{\alpha}, \delta_{x_{\alpha}}-\delta_{y_{\alpha}}\right)_{\alpha \in \Gamma}$ sistema biortogonal nice, o conjunto $F:=\left\{f_{\alpha}, \alpha \in \Gamma\right\}$ é irredundante em $C(K)$, pois dado $\alpha \in \Gamma$, temos que $1=\left(\delta_{x_{\alpha}}-\delta_{y_{\alpha}}\right)\left(f_{\alpha}\right)=f_{\alpha}\left(x_{\alpha}\right)-f_{\alpha}\left(y_{\alpha}\right)$ e, em particular, $f_{\alpha}\left(x_{\alpha}\right) \neq f_{\alpha}\left(y_{\alpha}\right)$. Por outro lado, para todo $\beta \neq \alpha$, temos que $0=\left(\delta_{x_{\alpha}}-\delta_{y_{\alpha}}\right)\left(f_{\beta}\right)=$ $f_{\beta}\left(x_{\alpha}\right)-f_{\beta}\left(y_{\alpha}\right)$, ou seja, $f_{\beta}\left(x_{\alpha}\right)=f_{\beta}\left(y_{\alpha}\right)$. Assim, $f_{\alpha}$ separa os pontos $x_{\alpha}, y_{\alpha}$, enquanto nenhum elemento em $\overline{\left\langle f_{\beta}: \beta \neq \alpha\right\rangle}$ separa os pontos $x_{\alpha}, y_{\alpha}$. Logo, $f_{\alpha} \notin \overline{\left\langle f_{\beta}: \beta \neq \alpha\right\rangle}$. Concluímos portanto que o conjunto $F$ é irredundante em $C(K)$. Logo

$$
\operatorname{irr}_{B a A}(C(K)) \geq \operatorname{nbiort}_{2}(K) .
$$

Concluímos assim a demonstração.

Definição 4.6. Seja $X$ um espaço topológico. Definimos o spread de X por:

$$
s(X)=\sup \{|D|: D \subset X \text { e } D \text { é discreto em } X\} .
$$

Teorema 4.4 (Heindorff). Seja A uma álgebra de Boole e $K$ um espaço compacto. Então:

1. $\operatorname{irr}_{B A}(A) \leq \operatorname{nbiort}_{2}(S(A))$;

2. $\operatorname{nbiort}_{2}(K) \leq s\left(K^{2}\right)$.

Demonstração. 1. Da Proposição 4.2 temos que $\operatorname{irr}_{B A}(A) \leq \operatorname{irr}_{B a A}(C(S(A)))$ e do Teorema 4.3 segue-se que $\operatorname{irr}_{B a A}(C(S(A)))=\operatorname{nbiort}_{2}(S(A))$. Logo $\operatorname{irr}_{B A}(A) \leq \operatorname{nbiort}_{2}(S(A))$.

2. Seja $\left(f_{\alpha}, \delta_{x_{\alpha}}-\delta_{y_{\alpha}}\right)_{\alpha \in \kappa}$ um sistema biortogonal nice. Para cada $\alpha \in \kappa$ sejam $U_{\alpha}, V_{\alpha}$ abertos em $K$ tais que

- $\forall x \in U_{\alpha}\left(\left|f_{\alpha}(x)-f_{\alpha}\left(x_{\alpha}\right)\right|<\frac{1}{2}\right)$, 
- $\forall x \in V_{\alpha}\left(\left|f_{\alpha}(x)-f_{\alpha}\left(y_{\alpha}\right)\right|<\frac{1}{2}\right)$.

Então, $\left(x_{\alpha}, y_{\alpha}\right) \in U_{\alpha} \times V_{\alpha}$. Seja agora $\beta \neq \alpha$. Se $\left(x_{\beta}, y_{\beta}\right) \in U_{\alpha} \times V_{\alpha}$, então

$$
\begin{gathered}
\left|f_{\alpha}\left(x_{\beta}\right)-f_{\alpha}\left(y_{\beta}\right)\right|=\left|f_{\alpha}\left(x_{\beta}\right)-f_{\alpha}\left(x_{\alpha}\right)+f_{\alpha}\left(x_{\alpha}\right)-f_{\alpha}\left(y_{\alpha}\right)+f_{\alpha}\left(y_{\alpha}\right)-f_{\alpha}\left(y_{\beta}\right)\right| \geq \\
\geq\left|1+f_{\alpha}\left(x_{\beta}\right)-f_{\alpha}\left(x_{\alpha}\right)\right|-\left|f_{\alpha}\left(y_{\alpha}\right)-f_{\alpha}\left(y_{\beta}\right)\right| \geq 1-\left|f_{\alpha}\left(x_{\beta}\right)-f_{\alpha}\left(x_{\alpha}\right)\right|-\left|f_{\alpha}\left(y_{\alpha}\right)-f_{\alpha}\left(y_{\beta}\right)\right|>0 .
\end{gathered}
$$

Logo, $\left|f_{\alpha}\left(x_{\beta}\right)-f_{\alpha}\left(y_{\beta}\right)\right|>0$, uma contradição, pois $f_{\alpha}\left(x_{\beta}\right)-f_{\alpha}\left(x_{\beta}\right)=0$ para $\alpha \neq \beta$.

Assim, $\left\{\left(x_{\alpha}, y_{\alpha}\right): \alpha \in \kappa\right\}$ é discreto em $K \times K$. Portanto $\kappa \leq s\left(K^{2}\right)$. Mostramos assim que para todo sistema biortogonal nice de tamanho $\kappa$, temos que $\kappa \leq s\left(K^{2}\right)$. Concluímos portanto que nbiort $_{2}(K) \leq s\left(K^{2}\right)$.

Obteremos agora um teorema sobre irredundância para espaços de Banach da forma $C(K)$ análogo ao Teorema de Mckenzie (Teorema 4.5 abaixo) para álgebras de Boole.

Precisamos antes de algumas funções cardinais:

Definição 4.7. Seja X um espaço topológico. Definimos o $\pi$-peso de X por:

$$
\pi(X):=\min \{|B|: B \text { é uma } \pi \text {-base para } X\} .
$$

Definição 4.8. Sejam K um compacto Hausdorff e A uma álgebra de Boole. Definimos

$$
\operatorname{irrm}_{B a A}(K):=\min \{|F|: F \subset C(K) \text { é irredundante maximal }\},
$$

$e$

$$
\operatorname{irrm}_{B A}(A):=\min \{|B|: B \subset A \text { é irredundante maximal }\} .
$$

Para álgebras de Boole, temos o seguinte teorema devido a McKenzie:

Teorema 4.5 (McKenzie). Todo subconjunto irredundante maximal de uma álgebra de Boole gera um subconjunto denso.

Demonstração. Ver [14], Proposição 4.23, página 62.

Obtemos do Teorema de McKenzie o seguinte corolário:

Corolário 4.2. Para toda álgebra de Boole A, vale que

$$
\pi(S(A)) \leq \operatorname{irrm}_{B A}(A) .
$$

Obteremos agora um novo resultado análogo ao Corolário 4.2 no contexto de espaços de Banach $C(K)$.

Notação 4.1. Dados um compacto $K, F \subset C(K)$ e $N \subset \mathbb{R}$ definamos

$$
\langle F\rangle_{N}:=\left\{\sum_{i=1}^{j} \lambda_{j} f_{1}^{i} \cdots f_{n_{i}}^{i}: \forall i\left(\lambda_{i} \in N\right), \forall i, l\left(f_{i}^{l} \in F\right)\right\} .
$$

Assim, $\langle F\rangle_{N}$ é o conjunto formado pelas combinações lineares finitas com coeficientes em $N$ de produtos finitos de elementos de $F$.

No caso de $N=\mathbb{R}$, denotamos $\langle F\rangle_{\mathbb{R}}:=\langle F\rangle$.

Teorema 4.6. Seja K um compacto Hausdorff. Então:

$$
\pi(K) \leq \operatorname{irrm}_{B a A}(K) .
$$


Demonstração. Seja $F \subset C(K)$ um irredundante maximal. Consideremos $\langle F\rangle_{\mathbb{Q}}$ o conjunto de todas as combinações lineares finitas racionais de produtos finitos de $F$.

Definimos, para cada $g \in C(K)$ e $p \in \mathbb{Q}^{+}$, o aberto

$$
B(g, p):=g^{-1}(]-\infty,-p[\cup] p, \infty[)
$$

e seja

$$
B:=\left\{B(g, p): p \in \mathbb{Q}^{+}, g \in\langle F\rangle_{\mathbb{Q}}\right\}
$$

Temos que $|B| \leq|F|$.

Para concluirmos a demonstração, basta mostrarmos que $B$ é uma $\pi$-base para $K$.

Seja $A$ um aberto não vazio de $K$. Tomemos $f_{A} \in C(K)$ tal que $f_{A}$ se anula fora de $A$ e toma o valor 1 em um ponto de $A$.

- Se $f_{A} \in F$, então $f_{A} \in\langle F\rangle_{\mathbb{Q}}$ e $B\left(f_{A}, \frac{1}{2}\right)=f_{A}^{-1}(]-\infty,-\frac{1}{2}[\bigcup] \frac{1}{2}, \infty[) \in B$ é aberto não vazio e contido em $A$.

- Suponhamos então que $f_{A} \notin F$.

Pela maximalidade de $F$, devemos ter $f_{A} \in \overline{\langle F\rangle}$ ou deve existir $g \in F$ tal que $g \in \overline{\left\langle F \backslash\{g\} \cup\left\{f_{A}\right\}\right\rangle}$.

- Se $f_{A} \in \overline{\langle F\rangle}$, como $\langle F\rangle_{\mathbb{Q}}$ é denso em $\overline{\langle F\rangle}$, deve existir $g \in\langle F\rangle_{\mathbb{Q}}$ tal que $\left\|f_{A}-g\right\|_{\infty}<\frac{1}{2}$. Assim, para todo $x \in K \backslash A$, temos que $\left(\left|f_{A}(x)-g(x)\right|=|g(x)| \leq\left\|f_{A}-g\right\|_{\infty}<\frac{1}{2}\right)$ e, portanto,

$$
B\left(g, \frac{1}{2}\right)=g^{-1}(]-\infty,-\frac{1}{2}[\cup] \frac{1}{2}, \infty[) \subset A .
$$

Observemos que por hipótese, existe $x \in A$ tal que $f(x)=1$. Logo, $\left|f_{A}(x)-g(x)\right|=$ $|1-g(x)|<\frac{1}{2}$, e daí, $|g(x)|>\frac{1}{2}$. Portanto, o aberto $B\left(g, \frac{1}{2}\right)$ não é vazio.

- Suponhamos agora que existe $g \in F$ tal que $g \in \overline{\left\langle F \backslash\{g\} \cup\left\{f_{A}\right\}\right\rangle}$.

Assim, existem sequências $\left(b_{n}\right)_{n}$ em $\langle F \backslash\{g\}\rangle_{\mathbb{Q}}$ e $\left(c_{n}\right)_{n}$ em $\left\langle F \backslash\{g\} \cup\left\{f_{A}\right\}\right\rangle_{\mathbb{Q}}$ tais que a sequência $\left(b_{n}+c_{n} f_{A}\right)_{n}$ converge para $g$ em $C(K)$.

Afirmação 1: Dado $p \in \mathbb{Q}^{+}$, existe $n_{0} \in \omega$ tal que

$$
\forall n>n_{0}\left(B\left(g-b_{n}, p\right) \in B \wedge B\left(g-b_{n}, p\right) \subset A\right) .
$$

Demonstração. Por definição de convergência, existe $n_{0} \in \omega$ tal que para todo $n>n_{0}$ temos que

$$
\left\|g-\left(b_{n}+c_{n} f_{A}\right)\right\|=\sup _{x \in K}\left|g(x)-\left(b_{n}(x)+\left(c_{n} f_{A}\right)(x)\right)\right|<p .
$$

Em particular, se $x \in K \backslash A$ e $n>n_{0}$ (lembrando que $f$ se anula fora de $A$ ):

$$
\left|g(x)-b_{n}(x)\right|=\left|g(x)-b_{n}(x)-\left(c_{n} f\right)(x)\right| \leq|| g-\left(b_{n}+c_{n} f\right) \|<p .
$$

Assim, se $n>n_{0}$ temos que $B\left(g-b_{n}, p\right) \subset A$ e como $g-b_{n} \in\langle F\rangle_{\mathbb{Q}}$ segue-se que $B\left(g-b_{n}, p\right) \in B$.

Vamos determinar $n \in \omega$ e $q \in \mathbb{Q}$ de tal modo que $B\left(g-b_{n}, p\right) \neq \emptyset$.

Afirmação 2: Existe $q \in \mathbb{Q}^{+}$tal que para todo $n_{0} \in \omega$ existe $n>n_{0}$ tal que $B\left(g-b_{n}, q\right)$ é não vazio.

Demonstração. Como $F$ é irredundante, a sequência de pontos $\left(b_{n}\right)_{n}$ não pode convergir para $g$ em $C(K)$. Logo, deve existir $\epsilon>0$ tal que para todo $n_{0} \in \omega$ existe $n>n_{0}$ tal 
que

$$
\sup _{x \in K}\left|g(x)-b_{n}(x)\right|>\epsilon .
$$

Assim, deve existir $y \in K$ tal que $\left|g(y)-b_{n}(y)\right|=\sup _{x \in K}\left|g(x)-b_{n_{1}}(x)\right|>\epsilon$.

Seja $q \in \mathbb{Q}^{+}$tal que $q<\epsilon$. Logo, $y \in B\left(g-b_{n}, q\right)$ e assim, $B\left(g-b_{n}, q\right)$ é não vazio.

Tomemos agora $q \in \mathbb{Q}^{+}$com a propriedade da Afirmação 2. Pela Afirmação 1, existe $n_{0} \in \omega$ tal que

$$
\forall n>n_{0}\left(B\left(g-b_{n}, q\right) \in B \wedge B\left(g-b_{n}, q\right) \subset A\right) .
$$

Pela escolha de $q$, existe $n_{1}>n_{0}$ tal que $B\left(g-b_{n_{1}}, q\right) \neq \emptyset$. Logo,

$$
\emptyset \neq B\left(g-b_{n_{1}}, q\right) \subset A .
$$

Com o Teorema 4.6, obtemos a seguinte desigualdade para espaços compactos:

Corolário 4.3. Para um compacto Hausdorff $K$ vale que

$$
\pi(K) \leq s\left(K^{2}\right)
$$

Demonstração. Segue-se do Teorema 4.6 que $\pi(K) \leq \operatorname{irrm}_{B a A}(K) \leq \operatorname{irr}_{B a A}(K)$ e do Teorema 4.4 temos que $\operatorname{irr}_{B a A}(K)=$ nbiort $_{2}(K)$.

Além disso, pelo Teorema 4.6, temos que nbiort $_{2}(K) \leq s\left(K^{2}\right)$. Assim segue-se que $\pi(K) \leq$ $s\left(K^{2}\right)$.

Observamos que a desigualdade obtida no Corolário 4.3 é também uma consequência do Teorema 3 de [13]. 


\section{Capítulo 5}

\section{Um espaço de Ostaszewski sob o Princípio Diamante $\diamond$}

O objetivo deste capítulo é a construção de um espaço compacto Hausdorff disperso cujas potências finitas são hereditariamente separáveis, ou seja, um espaço compacto satisfazendo as condições do enunciado do Teorema 3.10. Como mencionado no final da Seção 3.3, a construção de um compacto com estas propriedades não pode ser absoluta, ou seja, necessitamos de um axioma adicional à teoria de $Z F C$. Usaremos aqui o Princípio $\diamond$. O primeiro a obter um compacto $K$ satisfazendo as propriedades do Teorema 3.10 foi K. Kunen, assumindo a Hipótese do Contínuo. A construção de K. Kunen pode ser encontrada em [21]. O espaço que construiremos aqui é uma versão do espaço construído por A. J. Ostaszewski em [22] assumindo o Princípio $\diamond$.

Utilizaremos o Princípio $\diamond$ para a construção de uma álgebra de Boole $A$ superatômica, de tal modo que o respectivo espaço de Stone $S(A)$ da álgebra seja o compacto procurado. O método de construção seguira as ideias contidas no artigo [1]. Por indução transfinita, construiremos uma família crescente $\left(A_{\alpha}\right)_{\alpha<\omega_{1}}$ formada por subálgebras de Boole da álgebra $\wp(\mathbb{N})$ e definiremos $A$ como a união da família $\left(A_{\alpha}\right)_{\alpha<\omega_{1}}$. Para um ordinal limite $\gamma<\omega_{1}$, definiremos $A_{\gamma}$ como a união das álgebras anteriores. Para a construção de $A_{\gamma+1}$, dada a existência da álgebra $A_{\gamma}$, faremos uma extensão simples da álgebra $A_{\gamma}$. Assim, a primeira seção do capítulo trata exclusivamente de extensões simples. Na Seção 5.2, construímos uma álgebra de Boole superatômica em $Z F C$. Tal construção é um protótipo da construção da álgebra $A$. Finalmente, na Seção 5.3 , construímos a álgebra $A$ usando o Princípio $\diamond$ e definimos a noção de espaço de Ostaszewski.

\subsection{Extensões simples de álgebras enumeráveis}

Seja $A$ um corpo de conjuntos de $\mathbb{N}$ enumerável, $\operatorname{com} \operatorname{Fin}(\mathbb{N}) \subset A$ e $\mathfrak{u}$ um ultrafiltro não principal de $A$.

Definimos a seguinte ordem parcial:

$$
\mathbb{P}(A, \mathfrak{u}):=\{(a, b): a \cup b \notin \mathfrak{u} \wedge a \cap b=\emptyset\},
$$

ordenado por

$$
\left(a_{1}, b_{1}\right) \leq\left(a_{2}, b_{2}\right) \text { se, e somente se, } a_{2} \subset a_{1} \wedge b_{2} \subset b_{1} .
$$

Construiremos uma extensão simples $\left\langle A \cup\left\{x_{G}\right\}\right\rangle$ de $A$, adicionando um elemento $x_{G}$ que será construído através de um filtro $G$ da ordem parcial $\mathbb{P}(A, \mathfrak{u})$.

Definição 5.1. Se $G$ é um filtro em $\mathbb{P}(A, \mathfrak{u})$, definamos $x_{G}:=\bigcup\{a: \exists b((a, b) \in G\}$.

O filtro $G$ escolhido terá a propriedade de intersectar uma família enumerável de subconjuntos densos de $\mathbb{P}(A, \mathfrak{u})$ e esses conjuntos densos serão definidos de tal modo a fornecer informações sobre o espaço de Stone da extensão. 
Primeiramente, queremos construir a extensão da álgebra $A$ de tal modo que o espaço de Stone da extensão seja obtido do espaço de Stone de $A$ pela bifurcação do ultrafiltro $\mathfrak{u}$, ou seja, pela divisão do ultrafiltro $\mathfrak{u}$ em dois ultrafiltros distintos.

Assim, começamos definindo subconjuntos densos de $\mathbb{P}(A, \mathfrak{u})$ com tal finalidade.

Primeiramente, obteremos uma relação entre o elemento $\mathbb{N} \backslash x_{G}$ e o filtro $G$.

Lema 5.1. Seja $n \in \mathbb{N}$. Então

$$
D_{n}^{1}=\{(a, b) \in \mathbb{P}(A, \mathfrak{u}): n \in a \cup b\}
$$

é denso em $\mathbb{P}(A, \mathfrak{u})$.

Demonstração. Seja $(a, b) \in \mathbb{P}(A, \mathfrak{u})$. Suponhamos que $(a, b) \notin D_{n}^{1}$.

Consideremos $(a \cup\{n\}, b)$. Como $\mathfrak{u}$ não é principal, segue-se que $\mathfrak{u}$ não contém conjuntos finitos. Logo, como $a \cup b \notin \mathfrak{u}$, segue-se que $a \cup b \cup\{n\} \notin \mathfrak{u}$. Assim, $(a \cup\{n\}, b) \in D_{n}^{1}$ e $(a \cup\{n\}, b) \leq(a, b)$.

Definição 5.2. Nas condições do lema acima, definimos

$$
\mathcal{E}^{1}(A, \mathfrak{u}):=\left\{D_{n}^{1}: n \in \omega\right\} .
$$

Lema 5.2. Seja $G$ um filtro em $\mathbb{P}(A, \mathfrak{u})$ que intersecta cada elemento da família $\mathcal{E}^{1}(A, \mathfrak{u})$. Então,

$$
\mathbb{N} \backslash x_{G}=\bigcup\{b: \exists a((a, b) \in G)\} .
$$

Demonstração. Usando a definição de $x_{G}$, temos que

$$
\mathbb{N} \backslash x_{G}=\bigcap\{\mathbb{N} \backslash a: \exists b((a, b) \in G)\} .
$$

Vamos primeiramente mostrar que $\bigcup\{b: \exists a((a, b) \in G)\} \subset \mathbb{N} \backslash x_{G}$.

Fixemos $(a, b) \in G$. Tomemos agora $\left(a^{\prime}, b^{\prime}\right) \in G$. Por hipótese, existe $\left(a^{\prime \prime}, b^{\prime \prime}\right) \in G$ tal que

$$
\left(a^{\prime \prime}, b^{\prime \prime}\right) \leq\left(a^{\prime}, b^{\prime}\right),(a, b) .
$$

Então $b \subset b^{\prime \prime} \subset \mathbb{N} \backslash a^{\prime \prime} \subset \mathbb{N} \backslash a^{\prime}$, ou seja $b \subset \mathbb{N} \backslash a^{\prime}$. Concluímos portanto que $b \subset \mathbb{N} \backslash a^{\prime}$ para todo $\left(a^{\prime}, b^{\prime}\right) \in G$. Logo,

$$
\bigcup\{b: \exists a((a, b) \in G)\} \subset \bigcap\{\mathbb{N} \backslash a: \exists b((a, b) \in G)\}=\mathbb{N} \backslash x_{G} .
$$

Mostraremos agora a inclusão oposta. Seja $n \in \mathbb{N} \backslash x_{G}$. Por hipótese, $D_{n} \cap G \neq \emptyset$. Seja $(a, b) \in$ $D_{n} \cap G$. Por 5.1.1, temos que $n \in \mathbb{N} \backslash a$ e, daí, $n \in b$. Logo, $n \in \bigcup\{b: \exists a((a, b) \in G\}$. Assim,

$$
\mathbb{N} \backslash x_{G} \subset \bigcup\{b: \exists a((a, b) \in G\} .
$$

Quaremos que o ultrafiltro $\mathfrak{u}$ em $A$ seja bifurcado em dois ultrafiltros distintos na extensão $\left\langle A \cup\left\{x_{G}\right\}\right\rangle$. Para isso, é suficiente que $\mathfrak{u} \cup\left\{x_{G}\right\}$ e $\mathfrak{u} \cup\left\{\mathbb{N} \backslash x_{G}\right\}$ tenham a p.i.f. Construiremos a seguir uma família de densos de tal modo que se $G$ intersecta cada elemento dessa família, então $\mathfrak{u} \cup\left\{x_{G}\right\}$ e $\mathfrak{u} \cup\left\{\mathbb{N} \backslash x_{G}\right\}$ tem a p.i.f.

Lema 5.3. Seja $c \in \mathfrak{u}$. Então

$$
D_{c}^{2}:=\{(a, b) \in \mathbb{P}(A, \mathfrak{u}): a \cap c \neq \emptyset \wedge b \cap c \neq \emptyset\},
$$

é denso em $\mathbb{P}(A, \mathfrak{u})$. 
Demonstração. Seja $(a, b) \in \mathbb{P}(A, \mathfrak{u})$. Como u é não principal e $\mathbb{N} \backslash(a \cup b) \in \mathfrak{u}$ segue-se que $c \backslash(a \cup b)$ é infinito. Seja então $n_{1} \neq n_{2} \in c \backslash(a \cup b)$ e observemos que $\left(a \cup\left\{n_{1}\right\}, b \cup\left\{n_{2}\right\}\right) \in \mathbb{P}(A, \mathfrak{u})$. Temos portanto que $\left(a \cup\left\{n_{1}\right\}, b \cup\left\{n_{2}\right\}\right) \in D_{c}^{2}$ e é extensão de $(a, b)$.

Definição 5.3. Nas condições do lema acima, definimos

$$
\mathcal{E}^{2}(A, \mathfrak{u}):=\left\{D_{c}^{2}: c \in \mathfrak{u}\right\} .
$$

Lema 5.4. Seja $G$ um filtro em $\mathbb{P}(A, \mathfrak{u})$ tal que $G$ intersecta cada elemento da família

$$
\mathcal{E}^{1}(A, \mathfrak{u}) \cup \mathcal{E}^{2}(A, \mathfrak{u}) .
$$

Então $\mathfrak{u} \cup\left\{x_{G}\right\}$ e $\mathfrak{u} \cup\left\{\mathbb{N} \backslash x_{G}\right\}$ tem p.i.f.

Demonstração. Vamos mostrar que $\mathfrak{u} \cup\left\{x_{G}\right\}$ tem a p.i.f. A demostração que $\mathfrak{u} \cup\left\{\mathbb{N} \backslash x_{G}\right\}$ tem a p.i.f é análoga.

Suponhamos por absurdo que $\mathfrak{u} \cup\left\{x_{G}\right\}$ não tem a p.i.f. Então existe $c \in \mathfrak{u}$ tal que

$$
c \cap x_{G}=\emptyset .
$$

Por outro lado, como $G \cap D_{c}^{2} \neq \emptyset$, seja $(a, b) \in G \cap D_{c}^{2}$. Assim, $c \cap a \neq \emptyset$ e, portanto, $\emptyset \neq c \cap a \subset c \cap x_{G}$, o que é uma contradição com 5.1.2.

Corolário 5.1. Seja $G$ um filtro em $\mathbb{P}(A, \mathfrak{u})$ tal que $G$ intersecta cada elemento de $\mathcal{E}^{1}(A, \mathfrak{u}) \cup$ $\mathcal{E}^{2}(A, \mathfrak{u})$. Então existem exatamente dois ultrafiltros $\mathfrak{u}_{1}, \mathfrak{u}_{2} \in S\left(\left\langle A \cup\left\{x_{G}\right\}\right\rangle\right)$ tais que

$$
\mathfrak{u}_{1} \cap A=\mathfrak{u}=\mathfrak{u}_{2} \cap A .
$$

Demonstração. A existência de dois ultrafiltros é dada pelo lema anterior, e o fato de que existem apenas dois segue do fato que homomorfismos entre álgebras de Boole que coincidem num conjunto gerador são iguais.

Definição 5.4. Nas condições do corolário acima, definimos $\mathfrak{u}_{1}$ como o ultrafiltro gerado por $\mathfrak{u} \cup\left\{x_{G}\right\}$ e definimos $\mathfrak{u}_{2}$ como o ultrafiltro gerado por $\mathfrak{u} \cup\left\{\mathbb{N} \backslash x_{G}\right\}$.

Os resultados anteriores nos fornecem condições suficientes para que o ultrafiltro $\mathfrak{u}$ seja bifurcado em dois ultrafiltros distintos na extensão.

Obteremos agora resultados que garantem que apenas o ultrafiltro $\mathfrak{u}$ é bifurcado na extensão.

Lema 5.5. Seja $c \in A \backslash$ u. Então

$$
D_{c}^{3}=\{(a, b): c \subset a \cup b\}
$$

é denso em $\mathbb{P}(A, \mathfrak{u})$.

Demonstração. Seja $(a, b) \in \mathbb{P}(A, \mathfrak{u})$. Observe que $(a \cup(c \backslash b), b) \in \mathbb{P}(A \mathfrak{u})$. Então $(a \cup(c \backslash b), b) \in D_{c}^{3}$ e é extensão de $(a, b)$.

Definição 5.5. Nas condições do lema acima, definimos

$$
\mathcal{E}^{3}(A, \mathfrak{u}):=\left\{D_{c}^{3}: c \in A \backslash \mathfrak{u}\right\} .
$$

Lema 5.6. Seja $G$ um filtro em $\mathbb{P}(A, \mathfrak{u})$ que $G$ intersecta cada elemento de

$$
\mathcal{E}^{1}(A, \mathfrak{u}) \cup \mathcal{E}^{2}(A, \mathfrak{u}) \cup \mathcal{E}^{3}(A, \mathfrak{u}) .
$$

Se $\mathfrak{v} \in S(A) \backslash\{\mathfrak{u}\}$, então ou

$$
\mathfrak{v} \cup\left\{x_{G}\right\} \text { não tem a p.i.f ou } \mathfrak{v} \cup\left\{\mathbb{N} \backslash x_{G}\right\} \text { não tem pif . }
$$


Observação. Um dos conjuntos acima deve ter p.i.f, pois $\mathfrak{v}$ tem p.i.f e, portanto, pode ser estendido a um ultrafiltro em $S\left(\left\langle A \cup\left\{x_{G}\right\}\right\rangle\right)$ e esse ultrafiltro deve possuir $x_{G}$ ou $\mathbb{N} \backslash x_{G}$.

Demonstração. Seja $G$ um filtro em $\mathbb{P}(A, \mathfrak{u})$ como no enunciado.

Se $\mathfrak{v} \neq \mathfrak{u}$, então existe $c \in \mathfrak{v} \backslash \mathfrak{u}$. Por hipótese, existe $(a, b) \in D_{c}^{3} \cap G$ e, portanto, $c \subset a \cup b$. Assim, $c=(c \cap a) \cup(c \cap b) \in \mathfrak{v}$. Como $\mathfrak{v}$ é ultrafiltro e, portanto,é um filtro primo, devemos ter $c \cap a \in \mathfrak{v}$ ou $c \cap b \in \mathfrak{v}$.

Se $c \cap a \in \mathfrak{v}$, como $c \cap a \subset a \subset x_{G}$ temos que, $(c \cap a) \cap\left(\mathbb{N} \backslash x_{G}\right)=\emptyset$. Logo, $\mathfrak{v} \cup\left\{\mathbb{N} \backslash x_{G}\right\}$ não tem a p.i.f.

Se $c \cap b \in \mathfrak{v}$, como $c \cap b \subset b \subset \mathbb{N} \backslash x_{G}$ temos que, $(c \cap b) \cap\left(x_{G}\right)=\emptyset$. Logo, $\mathfrak{v} \cup\left\{x_{G}\right\}$ não tem a p.i.f.

Do lema, segue-se que se $\mathfrak{v}$ é ultrafiltro distinto de $\mathfrak{u}$, então $v$ não é bifurcado na extensão.

Definição 5.6. Seja $G$ um ultrafiltro que intersecta cada membro da família de densos $\mathcal{E}^{1}(A, \mathfrak{u}) \cup$ $\mathcal{E}^{2}(A, \mathfrak{u}) \cup \mathcal{E}^{3}(A, \mathfrak{u})$.

- Dado $\mathfrak{v} \in S(A) \backslash\{\mathfrak{u}\}$, denotaremos por $\langle\mathfrak{v}\rangle$ o único ultrafiltro em $S\left(\left\langle A \cup\left\{x_{G}\right\}\right\rangle\right)$ que contêm $\mathfrak{v}$.

- Dado $D \subset S(A) \backslash\{\mathfrak{u}\}$, denotaremos por $D^{\prime}$ o conjunto $\{\langle\mathfrak{v}\rangle: \mathfrak{v} \in D\}$.

Segue dos resultados acima, que se $G$ é filtro que intersecta cada elemento da família

$$
\mathcal{E}^{1}(A, \mathfrak{u}) \cup \mathcal{E}^{2}(A, \mathfrak{u}) \cup \mathcal{E}^{3}(A, \mathfrak{u}),
$$

então o ultrafiltro $\mathfrak{u}$ é bifurcado em dois ultrafiltros distintos e todo ultrafiltro distinto de $\mathfrak{u}$ em $S(A)$ não é bifurcado na extensão. A visualização do espaço $S\left(\left\langle A \cup\left\{x_{G}\right\}\right\rangle\right)$ como sendo obtido do espaço $S(A)$ por simples bifurcação do ultrafiltro $\mathfrak{u}$ fica mais clara com o seguinte lema:

Lema 5.7. Seja $G$ um filtro em $\mathbb{P}(A, \mathfrak{u})$ que intersecta a família de densos

$$
\mathcal{E}^{1}(A, \mathfrak{u}) \cup \mathcal{E}^{2}(A, \mathfrak{u}) \cup \mathcal{E}^{3}(A, \mathfrak{u}) .
$$

Então a aplicação $\varphi: S(A) \backslash\{\mathfrak{u}\} \rightarrow S\left(\left\langle A \cup\left\{x_{G}\right\}\right\rangle\right) \backslash\left\{\mathfrak{u}_{1}, \mathfrak{u}_{2}\right\}$ dada por $\varphi(\mathfrak{v})=\langle\mathfrak{v}\rangle$ é um homeomorfismo.

Demonstração. Consideremos a aplicação $\varphi: S(A) \backslash\{\mathfrak{u}\} \rightarrow S\left(\left\langle A \cup\left\{x_{G}\right\}\right\rangle\right) \backslash\left\{\mathfrak{u}_{1}, \mathfrak{u}_{2}\right\}$ dada por $\varphi(\mathfrak{v})=\langle\mathfrak{v}\rangle$. Pelo Lema 5.6 a aplicação $\varphi$ está bem definida e é bijetora. Vamos mostrar agora que $\varphi$ é contínua.

Dado $\mathfrak{v} \in S(A) \backslash \mathfrak{u}$, tomemos $V=s\left(\left(a \cap x_{G}\right) \cup\left(b \backslash x_{G}\right)\right)$ uma vizinhança de $\langle\mathfrak{v}\rangle$ em $S\left(\left\langle A \cup\left\{x_{G}\right\}\right\rangle\right)$. Como vé um ultrafiltro, devemos ter $a \cap x_{G} \in\langle\mathfrak{v}\rangle$ ou $b \backslash x_{G} \in\langle\mathfrak{v}\rangle$.

- Suponha que $a \cap x_{G} \in\langle\mathfrak{v}\rangle$ e seja $c \in \mathfrak{v} \backslash \mathfrak{u}$. Por hipótese, $G$ intersecta cada elemento da família $\mathcal{E}^{3}(A, \mathfrak{u})$ e, portanto, $G \cap D_{c}^{3} \neq \emptyset$. Seja $(d, e) \in G \cap E_{c}$. Então $c=(c \cap d) \cup(c \cap e) \in \mathfrak{v}$. Como $\mathfrak{v}$ é ultrafiltro e estamos supondo que $x_{G} \in\langle\mathfrak{v}\rangle$, segue-se que $c \cap d \in \mathfrak{v}$. Logo, $c \cap d \cap a \in \mathfrak{v}$. Consideremos então a vizinhança $s(c \cap d \cap a)$ de $\mathfrak{v}$ em $S(A)$ e vejamos que $\varphi(s(c \cap d \cap a)) \subset V$.

Suponhamos que $\mathfrak{v}^{\prime} \in s(c \cap d \cap a)$. Logo, $c \cap d \cap a \in \mathfrak{v}^{\prime}$, e como $a \cap x_{G} \geq c \cap d \cap a$ e $c \cap d \cap a \in \mathfrak{v}^{\prime}$, segue-se que $\left\langle\mathfrak{v}^{\prime}\right\rangle \in s\left(a \cap x_{G}\right)$. Portanto $\varphi(s(c \cap d \cap a)) \subset s\left(a \cap x_{G}\right) \subset s\left(\left(a \cap x_{G}\right) \cup\left(b \backslash x_{G}\right)\right)=V$. Concluímos assim que $\varphi$ é contínua em vo

- $\mathrm{O}$ caso em que $b \backslash x_{G} \in\langle\mathfrak{v}\rangle$ é análogo ao caso anterior.

Finalmente, consideremos a aplicação $\varphi^{-1}$. Temos que $\varphi^{-1}(s(a))=s(a)$ para todo $a \in A$. Logo, $\varphi^{-1}$ é contínua.

Concluímos assim que $\varphi$ é um homeomorfismo. 
Como mencionado na introdução do capítulo, queremos construir uma álgebra de Boole $A$ de tal modo que seu espaço de Stone $S(A)$ satisfaça as condições do enunciado do Teorema 3.10. Em particular, precisamos que as potências finitas de $S(A)$ sejam hereditariamente separáveis. O resto desta seção é voltado para a construção de densos em $\mathbb{P}(A, \mathfrak{u})$ relacionados à densidade hereditária das potências finitas de $S(A)$.

Começamos com alguns lemas que serão frequentemente usados quando trabalharmos com sistemas fundamentais de vizinhanças de elementos no espaço de Stone da extensão $\left\langle A \cup\left\{x_{G}\right\}\right\rangle$ e depois passaremos às correspondentes versões para as potências finitas.

Lema 5.8. Seja $G$ filtro em $\mathbb{P}(A, \mathfrak{u})$ que intersecta a família de densos

$$
\mathcal{E}^{1}(A, \mathfrak{u}) \cup \mathcal{E}^{2}(A, \mathfrak{u}) \cup \mathcal{E}^{3}(A, \mathfrak{u}) .
$$

$S e \Gamma \subset \mathfrak{u}$ é tal que $\{s(a): a \in \Gamma\}$ é um sistema fundamental de vizinhanças de $\mathfrak{u}$ em $S(A)$ então:

- $\left\{s\left(a \cap x_{G}\right): a \in \Gamma\right\}$ é um sistema fundamental de vizinhanças de $\mathfrak{u}_{1}$ em $S\left(\left\langle A \cup\left\{x_{G}\right\}\right\rangle\right) e$

- $\left\{s\left(a \backslash x_{G}\right): a \in \Gamma\right\}$ é um sistema fundamental de vizinhanças de $\mathfrak{u}_{2}$ em $S\left(\left\langle A \cup\left\{x_{G}\right\}\right\rangle\right)$.

Demonstração. Vamos demonstrar que $\left\{s\left(a \cap x_{G}\right): a \in \Gamma\right\}$ é um sistema fundamental de vizinhanças de $\mathfrak{u}_{1}$ em $S\left(\left\langle A \cup\left\{x_{G}\right\}\right\rangle\right)$. O outro caso é análogo.

Seja $b \in\left\langle A \cup\left\{x_{G}\right\}\right\rangle$ tal que $\mathfrak{u}_{1} \in s(b)$. Assim, $b \in \mathfrak{u}_{1}$. Pelo Teorema da Forma Normal 1.11, temos que $b=\left(a \cap x_{G}\right) \cup\left(c \backslash x_{G}\right)$ para certos $a, c \in A$.

Como $\mathfrak{u}_{1}$ é ultrafiltro, temos que $a \cap x_{G} \in \mathfrak{u}_{1}$ ou $c \backslash x_{G} \in \mathfrak{u}_{1}$. A segunda opção não pode ocorrer pois $x_{G} \in \mathfrak{u}_{1}$. Logo, $a \cap x_{G} \in \mathfrak{u}_{1}$ e então, $\mathfrak{u}_{1} \in s\left(a \cap x_{G}\right) \subset s(a)$. Observemos que $\mathfrak{u} \in s(a)$ e, portanto, existe $a^{\prime} \in \Gamma$ tal que $\mathfrak{u} \in s\left(a^{\prime}\right) \subset s(a)$. Assim obtemos que $\mathfrak{u}_{1} \in s\left(a^{\prime} \cap x_{G}\right) \subset s\left(a \cap x_{G}\right) \subset s(b)$.

Lema 5.9. Seja $D \subset S(A) \backslash\{\mathfrak{u}\}$ tal que $\mathfrak{u} \in \bar{D}$. Então existe uma família enumerável de densos $\mathcal{C}$ tal que se $G$ é um filtro em $\mathbb{P}(A, \mathfrak{u})$ e $G$ intersecta os densos da familia

$$
\mathcal{E}^{1}(A, \mathfrak{u}) \cup \mathcal{E}^{2}(A, \mathfrak{u}) \cup \mathcal{E}^{3}(A, \mathfrak{u}) \cup \mathcal{C}
$$

então $\mathfrak{u}_{1}, \mathfrak{u}_{2} \in \overline{D^{\prime}}$ em $S\left(\left\langle A \cup\left\{x_{G}\right\}\right\rangle\right)$.

Demonstração. Como a álgebra $A$ é enumerável, segue que o espaço de Stone de $A$ tem peso enumerável. Em particular, cada ponto do espaço $S(A)$ possui um sistema fundamental de vizinhanças enumerável. Seja $\left\{s\left(a_{n}\right): n \in \omega\right\}$ uma base de abertos fechados de $\mathfrak{u}$ decrescente. Definamos, para cada $n \in \omega$,

$$
C_{n}(D):=\left\{(a, b) \in \mathbb{P}(A, \mathfrak{u}): s(a) \cap s\left(a_{n}\right) \cap D \neq \emptyset \neq s(b) \cap s\left(a_{n}\right) \cap D\right\} .
$$

Afirmação. $C_{n}(D)$ é denso em $\mathbb{P}(A, \mathfrak{u})$ para todo $n \in \omega$.

Demonstração. Fixemos $n \in \omega$ e $\left(a^{\prime}, b^{\prime}\right) \in \mathbb{P}(A, \mathfrak{u})$.

Então $s\left(\mathbb{N} \backslash\left(a^{\prime} \cup b^{\prime}\right)\right)$ é vizinhança de $\mathfrak{u}$ e, portanto, $s\left(a_{n}\right) \cap s\left(\mathbb{N} \backslash\left(a^{\prime} \cup b^{\prime}\right)\right)$ é também uma vizinhança de $\mathfrak{u}$. Como $\mathfrak{u} \in \bar{D}$ e $\mathfrak{u} \notin D$, existe $\mathfrak{y}_{1} \in D$ tal que $\mathfrak{y}_{1} \in s\left(a_{n}\right) \cap s\left(\mathbb{N} \backslash\left(a^{\prime} \cup b^{\prime}\right)\right)$. Agora, como $S(A)$ é booleano, existe $a^{\prime \prime} \in A$ tal que $\mathfrak{y}_{1} \in s\left(a^{\prime \prime}\right) \subset s\left(a_{n}\right) \cap s\left(\mathbb{N} \backslash\left(a^{\prime} \cup b^{\prime}\right)\right)$ e $\mathfrak{u} \notin s\left(a^{\prime \prime}\right)$, ou seja, $\mathfrak{u} \in s\left(\mathbb{N} \backslash a^{\prime \prime}\right) \cap s\left(a_{n}\right) \cap s\left(\mathbb{N} \backslash\left(a^{\prime} \cup b^{\prime}\right)\right)$. Com a mesma argumentação, encontramos $\mathfrak{y}_{2} \in D$ e $b^{\prime \prime} \in A$ tais que $\mathfrak{y}_{2} \in s\left(b^{\prime \prime}\right) \subset s\left(\mathbb{N} \backslash a^{\prime \prime}\right) \cap s\left(a_{n}\right) \cap s\left(\mathbb{N} \backslash\left(a^{\prime} \cup b^{\prime}\right)\right)$ e $\mathfrak{u} \notin s\left(b^{\prime \prime}\right)$. Observamos assim que $\mathfrak{y}_{1}$ e $\mathfrak{y}_{2}$ são distintos elementos de $D$ e $a^{\prime \prime}, b^{\prime \prime}$ são elementos disjuntos de $A$ que satisfazem:

- $\mathfrak{y}_{1} \in s\left(a^{\prime \prime}\right), \mathfrak{y}_{2} \in s\left(b^{\prime \prime}\right)$,

- $s\left(a^{\prime \prime}\right), s\left(b^{\prime \prime}\right) \subset s\left(a_{n}\right) \cap s\left(\mathbb{N} \backslash\left(a^{\prime} \cup b^{\prime}\right)\right) \mathrm{e}$

- $\mathfrak{u} \notin s\left(a^{\prime \prime} \cup b^{\prime \prime}\right)$. 
Definamos agora $a=a^{\prime} \cup a^{\prime \prime}$ e $b=b^{\prime} \cup b^{\prime \prime}$. Temos que $a \cup b=a^{\prime} \cup b^{\prime} \cup a^{\prime \prime} \cup b^{\prime \prime}$ e, portanto, $a \cup b \notin \mathfrak{u}$, pois $a^{\prime}, a^{\prime \prime}, b^{\prime}, b^{\prime \prime} \notin \mathfrak{u}$. $\operatorname{Logo}(a, b) \in \mathbb{P}(A, \mathfrak{u})$. Por fim, observemos que $(a, b) \in C_{n}(D)$ e é extensão de $\left(a^{\prime}, b^{\prime}\right)$.

Definamos

$$
\mathcal{C}:=\left\{C_{n}(D): n \in \omega\right\} .
$$

Seja agora $G$ um filtro que satisfaz as condições do enunciado. Vamos mostrar que $\mathfrak{u}_{1} \in \overline{D^{\prime}}$. O caso $\mathfrak{u}_{2} \in \overline{D^{\prime}}$ é similar.

Pelo Lema 5.8, temos que

$$
\left\{s\left(a_{n} \cap x_{G}\right): n \in \omega\right\}
$$

é um sistema fundamental de vizinhanças de $\mathfrak{u}_{1}$. Assim, basta mostrarmos que para todo $n \in \omega$, existe $\langle\mathfrak{v}\rangle \in S\left(a_{n} \cap x_{G}\right)$ onde $\mathfrak{v} \in D$. Por hipótese, existe $(c, d) \in G \cap C_{n}(D)$. Em particular, pela definição de $C_{n}(D)$, existe $\mathfrak{v} \in D$ tal que $\mathfrak{v} \in s\left(a_{n}\right) \cap s(c)$. Então $a_{n}, c \in\langle\mathfrak{v}\rangle$. Como $c \subset x_{G}$, segue-se que $x_{G} \in\langle\mathfrak{v}\rangle$. Logo $\langle\mathfrak{v}\rangle \in s\left(a_{n} \cap x_{G}\right)$.

Definição 5.7. Nas condições do lema acima, definimos

$$
\mathcal{E}^{4}(A, \mathfrak{u}, D):=\left\{C_{n}(D): n \in \omega\right\} .
$$

Observemos que a definição da família $\mathcal{E}^{4}(A, \mathfrak{u}, D)$ depende de um subconjunto $D \subset S(A)$. Na construção da seção 5.3, utilizaremos o Princípio Diamante para escolhermos um determinado subconjunto $D_{a} l p h a \subset S\left(A_{\alpha}\right)$ em cada etapa do processo indutivo de construção.

O que faremos agora é generalizar a ideia do Lema 5.9 para as potências finitas do espaço de Stone da extensão.

Lema 5.10. Sejam $n \in \mathbb{N}$ e $D \subset S(A)^{n}$ onde cada elemento de $D$ tem todas as coordenadas distintas $e(\mathfrak{u}, \ldots, \mathfrak{u}) \in \bar{D}$. Então, para toda função $g:\{1, \ldots, n\} \rightarrow\{1,2\}$, existe uma familia enumerável de densos $\mathcal{F}^{g}(D)$ em $\mathbb{P}(A, \mathfrak{u})$ tal que se $G$ é um filtro que intersecta cada elemento da familia de densos

$$
\mathcal{E}^{1}(A, \mathfrak{u}) \cup \mathcal{E}^{2}(A, \mathfrak{u}) \cup \mathcal{E}^{3}(A, \mathfrak{u}) \cup \mathcal{F}^{g}(D),
$$

então

$$
\left(\mathfrak{u}_{g(1)}, \ldots, \mathfrak{u}_{g(n)}\right) \in \overline{D^{\prime}},
$$

onde $D^{\prime}=\left\{\left(\left\langle\mathfrak{v}_{1}\right\rangle, \ldots,\left\langle\mathfrak{v}_{n}\right\rangle\right):\left(\mathfrak{v}_{1}, \ldots, \mathfrak{v}_{n}\right) \in D\right\} \subset S\left(\left\langle A \cup\left\{x_{G}\right\}\right\rangle\right)^{n}$.

Demonstração. Seja $g:\{1, \ldots, n\} \rightarrow\{1,2\}$. Para cada $(a, b) \in \mathbb{P}(A, \mathfrak{u})$ e $j \in\{1, \ldots, n\}$ definamos

$$
\varphi(a, b, j)=\left\{\begin{array}{lll}
a & \text { se } & g(j)=1 \\
b & \text { se } & g(j)=2
\end{array}\right.
$$

Seja $\mathcal{V}_{\mathfrak{u}}=\left\{s\left(a_{n}\right): a_{n} \in \mathfrak{u}\right\}$ um sistema fundamental de vizinhanças para $\mathfrak{u}$

Dada $f:\{1, \ldots, n\} \rightarrow \mathbb{N}$, definamos

$$
F_{f}^{g}(D)=\left\{(a, b): D \cap\left[\prod_{j=1}^{n} s\left(a_{f(j)}\right) \cap s(\varphi(a, b, j))\right] \neq \emptyset\right\} .
$$

Afirmação. $F_{f}^{g}(D)$ é denso.

Demonstração. Seja $p=(c, d) \in \mathbb{P}(A, \mathfrak{u})$. Temos então que $(\mathfrak{u}, \ldots, \mathfrak{u}) \in s\left(\left(\bigcap_{j=1}^{n} a_{f(j)}\right) \backslash(c \cup d)\right)^{n}$. Por hipótese, existe $\left(v_{1}, \ldots, v_{n}\right) \in D \cap s\left(\left(\bigcap_{j=1}^{n} a_{f(j)}\right) \backslash(c \cup d)\right)^{n}$.

Sejam $c_{1}, \ldots, c_{n} \in A$ tais que

1) Para todo $j=1, \ldots, n$ temos que $c_{j} \in v_{j}$,

2) $c_{i} \cap c_{j}=\emptyset$, para $i \neq j$,

3) $\bigcup_{j=1}^{n} c_{j} \notin \mathfrak{u}$, 
4) para todo $j=1, \ldots, n$ temos que $c_{j} \subset\left(\bigcap_{j=1}^{n} a_{f(j)}\right) \backslash(c \cup d)$.

Seja $I:=\{j: g(j)=1\}$. Definamos por fim $q:=\left(c \cup \bigcup_{i \in I} c_{i}, d \cup \bigcup_{i \notin I} c_{i}\right)$. Primeiramente observamos que se $\left(c \cup \bigcup_{i \in I}\right) \cup\left(d \cup \bigcup_{i \notin I} c_{i}\right)=(c \cup d) \cup \bigcup_{j=1}^{n} c_{j} \in \mathfrak{u}$, como $\mathfrak{u}$ é ultrafiltro, devemos ter $c \cup d \in \mathfrak{u}$ ou $\bigcup_{j=1}^{n} c_{j} \in \mathfrak{u}$. A primeira opção não ocorre pois, $(c, d) \in \mathbb{P}(A, \mathfrak{u})$ e, portanto, $c \cup d \notin \mathfrak{u}$. A segunda opção não ocorre pela Propriedade 3). Logo, temos que $q \in \mathbb{P}(A, \mathfrak{u})$.

Vamos mostrar agora que $q \in F_{f}^{g}(D)$. Pelas propriedades 1) e 3), temos que $c_{j} \in a_{f(i)} \in v_{j}$, para todo $j=1, \ldots, n$.

Seja $i=1, \ldots, n$. Se $i \in I$, então $v_{i} \in s\left(c_{i}\right) \subset s\left(a_{f(i)}\right) \cap s\left(\bigcup_{i \in I} c_{i}\right) \subset s\left(a_{f(i)}\right) \cap s\left(c \cup \bigcup_{i \in I} c_{i}\right)=$ $s\left(a_{f(i)}\right) \cap s(\varphi(q, i))$. A última igualdade segue-se do fato que $g(i)=1 \mathrm{e}$, portanto, $\varphi(q, i)=c \bigcup_{i \in I} c_{i}$. De modo análogo, se $i \notin I$, então $v_{i} \in s\left(a_{f(i)}\right) \cap s(\varphi(q, i))$. Concluímos assim que $\left(v_{1}, \ldots, v_{n}\right) \in$ $D \cap\left[\prod_{i=1}^{n} s\left(a_{f(i)}\right) \cap s(\varphi(q, i))\right]$ e, portanto, $q \in F_{f}^{g}(D)$.

Assim, temos que $q \leq p$ e $q \in F_{f}^{g}(D)$.

Definamos

$$
\mathcal{F}^{g}(D):=\left\{F_{f}^{g}(D): f:\{1, \ldots, n\} \rightarrow \mathbb{N}\right\} .
$$

Seja $G$ satisfazendo as condições do enunciado.

Seja $V$ uma vizinhança de $\left(\mathfrak{u}_{g(1)}, \ldots, \mathfrak{u}_{g(n)}\right)$ em $S\left(\left\langle A \cup\left\{x_{G}\right\}\right\rangle\right)^{n}$. Como consequência do Lema 5.8 , podemos supor que existe $f:\{1, \ldots, n\} \rightarrow \mathbb{N}$ tal que

$$
\left(\mathfrak{u}_{g(1)}, \ldots, \mathfrak{u}_{g(n)}\right) \in \prod_{j=1}^{n} s\left(a_{f(j)} \cap h(j)\right),
$$

onde $h:\{1, \ldots, n\} \rightarrow\left\{x_{G}, \mathbb{N} \backslash x_{G}\right\}$ é dada por

$$
h(j)=\left\{\begin{array}{ccc}
x_{G} & \text { se } & g(j)=1 \\
\mathbb{N} \backslash x_{G} & \text { se } & g(j)=2
\end{array}\right.
$$

Por hipótese, existe $q=(a, b) \in G \cap F_{f}^{g}(D)$. Assim, existe $\left(v_{1}, \ldots, v_{n}\right) \in D \cap\left[\prod_{j=1}^{n} s\left(a_{f(j)}\right) \cap\right.$ $s(\varphi(a, b, j))]$.

Seja $j \in\{1, \ldots, n\}$.

- Se $g(j)=1$, então $\varphi(a, b, j)=a$. Logo $a_{f(j)}, a \in v_{j}$. Como $a \subset x_{G}$ segue-se que $x_{G} \in\left\langle v_{j}\right\rangle$. Além disso, como $g(j)=1$, segue-se que $h(j)=x_{G}$. Assim, $\left\langle v_{j}\right\rangle \in s\left(a_{f(j)} \cap h(j)\right)$.

- Se $g(j)=2$, então $\varphi(a, b, j)=b$. Logo $a_{f(j)}, b \in v_{j}$. Como $b \subset\left(\mathbb{N} \backslash x_{G}\right)$ segue-se que $\mathbb{N} \backslash x_{G} \in$ $\left\langle v_{j}\right\rangle$. Além disso, como $g(j)=2$, segue-se que $h(j)=\mathbb{N} \backslash x_{G}$. Assim, $\left\langle v_{j}\right\rangle \in s\left(a_{f(j)} \cap h(j)\right)$.

Concluímos assim que $\left(\left\langle v_{1}\right\rangle, \ldots,\left\langle v_{n}\right\rangle\right) \in V \cap D^{\prime}$.

Definição 5.8. Nas condições do lema acima definamos

$$
\mathcal{E}_{n}^{5}(A, \mathfrak{u}, D):=\bigcup\left\{\mathcal{F}^{g}(D): g:\{1, \ldots, n\} \rightarrow\{1,2\}\right\} .
$$

Lema 5.11. Seja $G$ um filtro em $\mathbb{P}(A, \mathfrak{u})$ que intersecta a familia de densos

$$
\mathcal{E}^{1}(A, \mathfrak{u}) \cup \mathcal{E}^{2}(A, \mathfrak{u}) \cup \mathcal{E}^{3}(A, \mathfrak{u}) .
$$

Temos que a aplicação $\varphi^{n}:(S(A) \backslash\{\mathfrak{u}\})^{n} \rightarrow\left(S\left(\left\langle A \cup\left\{x_{G}\right\}\right\rangle\right) \backslash\left\{\mathfrak{u}_{1}, \mathfrak{u}_{2}\right\}\right)^{n}$, dada por

$$
\varphi^{n}\left(v_{1}, \ldots, v_{n}\right)=\left(\left\langle v_{1}\right\rangle, \ldots,\left\langle v_{n}\right\rangle\right),
$$

é um homeomorfismo.

Demonstração. Consequência do Lema 5.7. 
Lema 5.12. Seja $G$ um filtro em $\mathbb{P}(A, \mathfrak{u})$ que intersecta a família de densos

$$
\mathcal{E}^{1}(A, \mathfrak{u}) \cup \mathcal{E}^{2}(A, \mathfrak{u}) \cup \mathcal{E}^{3}(A, \mathfrak{u}) .
$$

Consideremos $D \subset(S(A) \backslash\{\mathfrak{u}\})^{n}$ tal que $\left(v_{1}, \ldots, v_{n}\right) \in \bar{D}$, onde $v_{1}, \ldots, v_{n} \neq \mathfrak{u}$. Então $\left(\left\langle v_{1}\right\rangle, \ldots,\left\langle v_{n}\right\rangle\right) \in$ $\overline{D^{\prime}}$ em $S\left(\left\langle A \cup\left\{x_{G}\right\}\right\rangle\right)^{n}$.

Demonstração. Consequência do lema anterior observando que $\varphi^{n}(D)=D^{\prime}$.

Lema 5.13. Sejam $D \subset S(A)^{n}$, onde cada elemento de $D$ tem todas as coordenadas distintas, $e$ $h:\{1, \ldots, n\} \rightarrow S(A)$ tal que existem $I, J \neq \emptyset \operatorname{com}\{1, \ldots, n\}=I \cup J$ e $h_{\mid I}=\mathfrak{u}$ e $h(j) \neq \mathfrak{u}$ para todo $j \in J$.

Se $(h(1), \ldots, h(n)) \in \bar{D}$ em $S(A)^{n}$ então, para toda função $f:\{1, \ldots, n\} \rightarrow\{1,2\}$, existe uma família enumerável de densos $\mathcal{G}_{h}^{f}$ em $\mathbb{P}(A, \mathfrak{u})$ tal que se $G$ é um filtro em $\mathbb{P}(A, \mathfrak{u})$ que intersecta cada denso da família

$$
\mathcal{E}^{1}(A, \mathfrak{u}) \cup \mathcal{E}^{2}(A, \mathfrak{u}) \cup \mathcal{E}^{3}(A, \mathfrak{u}) \cup \mathcal{G}_{h}^{f},
$$

então

$$
(g(1), \ldots, g(n)) \in \overline{D^{\prime}}
$$

onde

$$
g(i)=\left\{\begin{array}{ccc}
\mathfrak{u}_{f(i)} & \text { se } & i \in I \\
\langle h(i)\rangle & \text { se } & i \in J
\end{array}\right.
$$

Demonstração. Fixemos $f:\{1, \ldots, n\} \rightarrow\{1,2\}$. Sejam $\mathcal{V}_{\mathfrak{u}}=\left\{s\left(a_{n}\right): a_{n} \in \mathfrak{u}\right\}$ um sistema fundamental de vizinhanças para $\mathfrak{u}$ e $\mathcal{V}_{j}=\left\{s\left(b_{n}^{j}\right): b_{n}^{j} \in h(j)\right\}$ uma base de $h(j)$ para $j \in I$.

Seja $l:\{1, \ldots, n\} \rightarrow \mathbb{N}$.

Para cada $(a, b) \in \mathbb{P}(A, \mathfrak{u})$ e $j \in\{1, \ldots, n\}$ definamos

$$
\varphi(a, b, j):=\left\{\begin{array}{ccc}
a \cap a_{l(j)} & \text { se } & j \in I \wedge f(j)=1 \\
b \cap a_{l(j)} & \text { se } & j \in I \wedge f(j)=2 \\
b_{l(j)}^{j} & \text { se } & j \in J .
\end{array}\right.
$$

Definamos

$$
G_{l}(D, h):=\left\{(a, b): D \cap \prod_{j=1}^{n} s(\varphi(a, b, j)) \neq \emptyset\right\} .
$$

Afirmação. $G_{l}(D, h)$ é denso em $\mathbb{P}(A, \mathfrak{u})$.

Demonstração. Seja $p=(y, z) \in \mathbb{P}(A, \mathfrak{u})$. Então $(h(1), \ldots, h(n)) \in \prod_{j=1}^{n} s\left(c_{j}\right)$, onde

$$
c_{j}=\left\{\begin{array}{ccc}
a_{l(j)} \backslash(y \cup z) & \text { se } & j \in I \\
b_{l(j)}^{j} & \text { se } & j \in J .
\end{array}\right.
$$

Por hipótese, existe $\left(v_{1}, \ldots, v_{n}\right) \in D \cap \prod_{j=1}^{n} s\left(c_{j}\right)$. Sejam $a_{1}, \ldots, a_{n} \in A$ tais que

1) $\forall j\left(a_{j} \in v_{j}\right)$,

2) $a_{j} \cap a_{i}=\emptyset$, para $i \neq j$,

3) $\bigcup_{j=1}^{n} a_{j} \notin \mathfrak{u}$,

4) $\forall j\left(a_{j} \subset c_{j}\right)$.

Definamos agora $q_{1}:=y \cup \bigcup_{j \in f^{-1}(\{1\})} a_{j}$ e $q_{2}:=z \cup \bigcup_{j \in f^{-1}(\{2\})} a_{j}$. Observemos que $q_{1} \cup q_{2}=$ $(y \cup z) \cup \bigcup_{j=1}^{n} a_{j}$. Como $y \cup z \notin \mathfrak{u}$ pois $p=(y, z) \in \mathbb{P}(A, \mathfrak{u})$ e $\bigcup_{j=1}^{n} a_{j} \notin \mathfrak{u}$ por 3$)$, do fato de ser $\mathfrak{u}$ um ultrafiltro, concluímos que $q_{1} \cup q_{2} \notin \mathfrak{u}$. Assim $q:=\left(q_{1}, q_{2}\right)$ é um elemento de $\mathbb{P}(A, \mathfrak{u})$. É fácil ver que $q \leq p$. Para concluirmos a demonstração da afirmação, basta mostrarmos que $q \in G_{l}(D, h)$. 
Para $j=1, \ldots, n$ temos que

$$
\varphi\left(q_{1}, q_{2}, j\right):=\left\{\begin{array}{ccc}
q_{1} \cap a_{l(j)} & \text { se } & j \in I \wedge f(j)=1 \\
q_{2} \cap a_{l(j)} & \text { se } & j \in I \wedge f(j)=2 \\
b_{l(j)}^{j} & \text { se } & j \in J .
\end{array}\right.
$$

Logo,

- se $j \in I$ e $f(j)=1$, então $v_{j} \in s\left(a_{j}\right) \subset s\left(q_{1}\right)$ e como $a_{j} \subset c_{j}=a_{l(j)} \backslash(y \cup z)$, temos que $v_{j} \in s\left(q_{1}\right) \cap s\left(a_{l(j)}\right)=s\left(q_{1} \cap a_{l(j)}\right)=s\left(\varphi\left(q_{1}, q_{2}, j\right)\right)$,

- se $j \in I$ e $f(j)=2$, então $v_{j} \in s\left(a_{j}\right) \subset s\left(q_{2}\right)$ e como $a_{j} \subset c_{j}=a_{l(j)} \backslash(y \cup z)$, temos que $v_{j} \in s\left(q_{2}\right) \cap s\left(a_{l(j)}\right)=s\left(q_{2} \cap a_{l(j)}\right)=s\left(\varphi\left(q_{1}, q_{2}, j\right)\right)$,

- se $j \in J$ então $v_{j} \in s\left(a_{j}\right)$ e como $a_{j} \subset c_{j}=b_{l(j)}^{j}$, temos que $v_{j} \in s\left(a_{j}\right) \subset s\left(b_{l(j)}^{j}\right)=$ $s\left(\left(\varphi\left(q_{1}, q_{2}, j\right)\right)\right.$.

Concluímos assim que $\left(v_{1}, \ldots, v_{n}\right) \in D \cap \prod_{j=1}^{n} s\left(\varphi\left(q_{1}, q_{2}, j\right)\right)$. Logo $q=\left(q_{1}, q_{2}\right)$ é um elemento de $G_{l}(D, h)$ e é uma extensão de $p$.

Definamos agora

$$
\mathcal{G}_{h}^{f}:=\left\{G_{l}(D, h): l:\{1, \ldots, n\} \rightarrow \mathbb{N}\right\} .
$$

Seja $G$ um filtro satisfazendo as condições do enunciado. Seja $V$ uma vizinhança de $(g(1), \ldots, g(n))$. Como consequência do Lema 5.8, podemos supor que existe $l:\{1, \ldots, n\} \rightarrow \mathbb{N}$ tal que

$$
g_{j} \in\left\{\begin{array}{ccc}
s\left(a_{l(j)} \cap x_{G}\right) & \text { se } & j \in I \wedge f(j)=1 \\
s\left(a_{l(j)} \backslash x_{G}\right) & \text { se } & j \in I \wedge f(j)=2 \\
s\left(b_{l(j)}^{j}\right) & \text { se } & j \in J .
\end{array}\right.
$$

Por hipótese, $G_{l}(D, h) \cap G \neq \emptyset$, logo existe $(a, b) \in G_{l}(D, h) \cap G$. Assim, existe

$$
\left(v_{1}, \ldots, v_{n}\right) \in D \cap \prod_{j=1}^{n} s(\varphi(a, b, j)) .
$$

Seja $j \in\{1, \ldots, n\}$.

- Se $j \in I$, então temos os seguintes casos:

- Se $f(j)=1$, então $\varphi(a, b, j)=a \cap a_{l(j)}$, logo $a \cap a_{l(j)} \in v_{j}$. Portanto, $a_{l(j)} \in v_{j}$ e $x_{G} \geq a \in v_{j}$. Logo, $\left\langle v_{j}\right\rangle \in s\left(a_{l(j)} \cap x_{G}\right)$.

- Se $f(j)=2$, então $\varphi(a, b, j)=b \cap a_{l(j)}$, $\operatorname{logo} b \cap a_{l(j)} \in v_{j}$. Portanto, $a_{l(j)} \in v_{j}$ e $\mathbb{N} \backslash x_{G} \geq b \in v_{j}$. Logo, $\left\langle v_{j}\right\rangle \in s\left(a_{l(j)} \backslash x_{G}\right)$.

- Se $j \in J$, então $\varphi(a, b, j)=b_{l(j)}^{j}, \operatorname{logo} b_{l(j)}^{j} \in v_{j}$. Portanto, $\left\langle v_{j}\right\rangle \in s\left(b_{l(j)}^{j}\right)$.

Concluímos assim que $\left(\left\langle v_{1}\right\rangle, \ldots,\left\langle v_{n}\right\rangle\right) \in V$.

Definição 5.9. Nas condições do lema acima, definamos

- $\chi(n, A, \mathfrak{u}):=\left\{h: h:\{1, \ldots, n\} \rightarrow S(A)\right.$ onde $\{1, \ldots, n\}=I \cup J, I, J \neq \emptyset, h_{\mid I}=\mathfrak{u}$ e $h(j) \neq$ $\mathfrak{u}$ para todo $j \in J\}$.

- $\mathcal{E}_{n}^{6}(A, \mathfrak{u}, D):=\bigcup\left\{G_{h}^{f}: h \in \chi(n, A, \mathfrak{u}) \wedge f:\{1, \ldots, n\} \rightarrow\{1,2\}\right\}$. 


\subsection{Uma álgebra superatômica não enumerável}

Nesta seção, faremos uma construção genérica de uma álgebra de Boole superatômica $A^{\prime}$.

Vamos construir uma sequência $\left(A_{\alpha}, \mathfrak{u}_{\alpha}, x_{\alpha}\right)_{\alpha<\omega_{1}}$ tal que, para cada $\alpha<\omega_{1}$, temos que $A_{\alpha}$ é uma subálgebra enumerável de $\wp(\mathbb{N})$ contendo $\operatorname{Fin} \operatorname{Cofin}(\mathbb{N}), \mathfrak{u}_{\alpha}$ é um ultrafiltro não principal de $A_{\alpha}$ e $x_{\alpha}$ é um subconjunto de $\mathbb{N}$.

- Seja $A_{0}=\operatorname{FinCofin}(\mathbb{N})$ e consideremos em $A_{0}$ o ultrafiltro não principal $\mathfrak{u}_{0}=\operatorname{Cofin}(\mathbb{N})$.

Consideremos agora $\alpha<\omega_{1}$.

- Se $\alpha=\beta+1$, para um ordinal $\beta<\omega_{1}$, consideremos a ordem parcial $\mathbb{P}\left(A_{\beta}, \mathfrak{u}_{\beta}\right)$. Seja $G \beta$ um filtro em $\mathbb{P}\left(A_{\beta}, \mathfrak{u}_{\beta}\right)$ que intersecta os densos da família:

$$
\mathcal{E}^{1}\left(A_{\beta}, \mathfrak{u}_{\beta}\right\} \cup \mathcal{E}^{2}\left(A_{\beta}, \mathfrak{u}_{\beta}\right) \cup \mathcal{E}^{3}\left(A_{\beta}, \mathfrak{u}_{\beta}\right) .
$$

Sejam $x_{\beta}=x_{G_{\beta}}$ como definido na seção anterior, $A_{\beta+1}=\left\langle A_{\beta} \cup\left\{x_{\beta}\right\}\right\rangle$ e seja $\mathfrak{u}_{\beta+1}$ o ultrafiltro em $A_{\beta+1}$ gerado por $\mathfrak{u}_{\beta} \cup\left\{x_{\beta}\right\}$, ou seja, $\mathfrak{u}_{\beta+1}=\left(\mathfrak{u}_{\beta}\right)_{1}$.

- Se $\alpha$ é um ordinal limite, definamos $A_{\alpha}=\bigcup_{\lambda<\alpha} A_{\lambda}$ e $\mathfrak{u}_{\alpha}=\bigcup_{\lambda<\alpha} \mathfrak{u}_{\lambda}$.

Definamos agora $A^{\prime}=\bigcup_{\alpha<\omega_{1}} A_{\alpha}$.

O próximo lema nos diz que para cada $\alpha<\omega_{1}$, o espaço de Stone da álgebra $A_{\alpha}$ é homeomorfo a um espaço compacto tendo como suporte o conjunto $[0, \alpha]$.

Lema 5.14. Para todo ordinal $\alpha<\omega_{1}$

$$
\begin{gathered}
S\left(A_{\alpha}\right)=\left\{n^{*}: n \in \omega\right\} \cup\left\{\left\langle\left\{x_{\beta}: \beta<\alpha\right\} \cup \operatorname{Cofin}(\mathbb{N})\right\rangle\right\} \cup \\
\cup\left\{\left\langle\left\{x_{\beta}: \beta<\gamma\right\} \cup\left\{\mathbb{N} \backslash x_{\gamma}\right\} \cup \operatorname{Cofin}(\mathbb{N})\right\rangle: \gamma<\alpha\right\} .
\end{gathered}
$$

Demonstração. Por indução em $\alpha<\omega_{1}$.

Suponha que $\alpha=\gamma+1$. Seja $\mathfrak{u}$ um ultrafiltro em $A_{\gamma+1}$. Então $\mathfrak{v}=\mathfrak{u} \cap A_{\gamma}$ é ultrafiltro em $S\left(A_{\gamma}\right)$. Por hipótese, temos que

$$
\begin{gathered}
S\left(A_{\gamma}\right)=\left\{n^{*}: n \in \omega\right\} \cup\left\{\left\langle\left\{x_{\beta}: \beta<\gamma\right\} \cup \operatorname{Cofin}(\mathbb{N})\right\rangle\right\} \cup \\
\cup\left\{\left\langle\left\{x_{\beta}: \beta<\xi\right\} \cup\left\{\mathbb{N} \backslash x_{\xi}\right\} \cup \operatorname{Cofin}(\mathbb{N})\right\rangle: \xi<\gamma\right\}
\end{gathered}
$$

- Se $\mathfrak{v}=n^{*}$ para algum $n \in \omega$, então $\mathfrak{u}=n^{*}$ em $A_{\gamma+1}$.

- Suponhamos que $\mathfrak{v}=\left\langle\left\{x_{\beta}: \beta<\gamma\right\} \cup \operatorname{Cofin}(\mathbb{N})\right\rangle=\mathfrak{u}_{\gamma}$. Pelo Lema 5.1, segue que $\mathfrak{u}_{\gamma}$ é bifurcado em $\left\langle\mathfrak{u}_{\gamma} \cup\left\{x_{\gamma}\right\}\right\rangle$ e $\left\langle\mathfrak{u}_{\gamma} \cup\left\{\mathbb{N} \backslash x_{\gamma}\right\}\right\rangle$. Assim, se $x_{\gamma} \in \mathfrak{u}$, então

$$
\mathfrak{u}=\left\langle\mathfrak{u}_{\gamma} \cup\left\{x_{\gamma}\right\}\right\rangle=\left\langle\left\{x_{\beta}: \beta<\gamma+1\right\} \cup \operatorname{Cofin}(\mathbb{N})\right\rangle .
$$

Caso contrário, temos que

$$
\mathfrak{u}=\left\langle\mathfrak{u}_{\gamma} \cup\left\{\mathbb{N} \backslash x_{\gamma}\right\}\right\rangle=\left\langle\left\{x_{\beta}: \beta<\gamma\right\} \cup\left\{\mathbb{N} \backslash x_{\gamma}\right\} \cup \operatorname{Cofin}(\mathbb{N})\right\rangle .
$$

- Se $\sqsubseteq=\left\langle\left\{x_{\beta}: \beta<\xi\right\} \cup\left\{\mathbb{N} \backslash x_{\xi}\right\} \cup \operatorname{Cofin}(\mathbb{N})\right\rangle$ para um $\xi<\gamma$, então pelo lema 5.6 o ultrafiltro $\sqsubseteq \neq \mathfrak{u}_{\gamma}$ não bifurca. Como $\left\langle\left\{x_{\beta}: \beta<\xi\right\} \cup\left\{\mathbb{N} \backslash x_{\xi}\right\} \cup \operatorname{Cofin}(\mathbb{N})\right\rangle \subset \mathfrak{u}$ segue que

$$
\mathfrak{u}=\left\langle\left\{x_{\beta}: \beta<\xi\right\} \cup\left\{\mathbb{N} \backslash x_{\xi}\right\} \cup \operatorname{Cofin}(\mathbb{N})\right\rangle .
$$

Suponhamos agora que $\alpha$ é limite. Seja $\mathfrak{u} \in S\left(A_{\alpha}\right)$. Então $\mathfrak{u}=\bigcup_{\gamma<\alpha}\left(\mathfrak{u} \cap A_{\gamma}\right)$.

Se para algum $\gamma<\alpha$ temos que $\mathfrak{u} \cap A_{\gamma}$ é principal, então claramente $\mathfrak{u}$ será principal em $S\left(A_{\alpha}\right)$. 
Se existe $\gamma<\alpha$ tal que $\mathfrak{u} \cap A_{\gamma}=\left\langle\left\{x_{\beta}: \beta<\xi\right\} \cup\left\{\mathbb{N} \backslash x_{\xi}\right\} \cup \operatorname{Cofin}(\mathbb{N})\right\rangle$ para algum $\xi<\gamma$, então o ultrafiltro $\mathfrak{u} \cap A_{\gamma}$ não é bifurcado nas extensões posteriores, logo devemos ter $\mathfrak{u}=\left\langle\left\{x_{\beta}\right.\right.$ : $\left.\beta<\xi\} \cup\left\{\mathbb{N} \backslash x_{\xi}\right\} \cup \operatorname{Cofin}(\mathbb{N})\right\rangle$.

Por último, se para todo $\gamma<\alpha$ tivermos que $\mathfrak{u} \cap A_{\gamma}=\left\langle\left\{x_{\beta}: \beta<\gamma\right\} \cup \operatorname{Cofin}(\mathbb{N})\right\rangle$, então $\mathfrak{u}=\left\langle\left\{x_{\beta}: \beta<\alpha\right\} \cup \operatorname{Cofin}(\mathbb{N})\right\rangle$.

Notação 5.1. Seja $\alpha<\omega_{1}$. Utilizaremos a seguinte notação:

- Para cada $n \in \omega$, denotaremos por $n^{* \alpha}$ o ultrafiltro $n^{*} \in S\left(A_{\alpha}\right)$.

- Para cada $\gamma<\alpha$, denotaremos por $\gamma^{* \alpha}$ o ultrafiltro

$\left\langle\left\{x_{\beta}: \beta<\gamma\right\} \cup\left\{\mathbb{N} \backslash x_{\gamma}\right\} \cup \operatorname{Cofin}(\mathbb{N})\right\rangle \in S\left(A_{\alpha}\right)$.

- Denotaremos por $\omega_{1}^{* \alpha}=\mathfrak{u}_{\alpha}=\left\langle\left\{x_{\beta}: \beta<\alpha\right\} \cup \operatorname{Cofin}(\mathbb{N})\right\rangle$.

Assim, pelo Lema 5.14, $S\left(A_{\alpha}\right)=\left\{n^{* \alpha}: n \in \omega\right\} \cup\left\{\gamma^{* \alpha}: \gamma<\alpha\right\} \cup\left\{\omega_{1}^{* \alpha}\right\}$.

Notação 5.2. No caso particular de $A^{\prime}=A_{\omega_{1}}=\bigcup_{\alpha<\omega_{1}} A_{\alpha}$, escreveremos simplesmente $\alpha$ no lugar de $\alpha^{* \omega_{1}}$ para cada $\alpha \leq \omega_{1}$. Assim, $S\left(A^{\prime}\right)=\left[0, \omega_{1}\right]$.

Com a notação acima, observamos que na construção da álgebra $A^{\prime}$ estamos, em cada passo $\alpha<\omega_{1}$, obtendo uma topologia sobre o conjunto $[0, \alpha]$.

Observação 5.1. Com as notações acima, dados $\delta<\beta<\alpha<\omega_{1}$ temos que

- $\beta^{* \alpha}=\beta \cap A_{\alpha}$.

- $\delta^{* \beta}=\delta^{* \alpha} \cap A_{\beta}$.

O principal resultado desta seção nos diz que a álgebra $A^{\prime}$ é superatômica (equivalentemente, o espaço $S\left(A^{\prime}\right)$ é disperso). Para demonstrarmos tal fato, precisamos antes do seguinte lema:

Lema 5.15. Consideremos a álgebra $A^{\prime}$. Seja $\alpha<\omega_{1}$ e consideremos o aberto $s\left(\mathbb{N} \backslash x_{\alpha}\right)$. Então $s\left(\mathbb{N} \backslash x_{\alpha}\right) \subset \alpha+1$.

Demonstração. De fato, seja $\mathfrak{u}$ ultrafiltro em $S\left(A^{\prime}\right)$ tal que $\mathfrak{u} \in s\left(\mathbb{N} \backslash x_{\alpha}\right)$, ou seja $\mathbb{N} \backslash x_{\alpha} \in \mathfrak{u}$. Pelo Lema 5.14, temos que $\mathfrak{u}=\gamma$ para algum $\gamma \leq \alpha$. Logo, $\mathfrak{u} \in \alpha+1$.

Teorema 5.1. $S\left(A^{\prime}\right)$ é um espaço disperso.

Demonstração. Seja $Y \subset S(A)$. Tomemos $\alpha=\min Y$. Pelo Lema 5.15, s(N $\left.\backslash x_{\alpha}\right) \subset \alpha+1, \operatorname{logo}$ $s\left(\mathbb{N} \backslash x_{\alpha}\right) \cap Y=\{\alpha\}$. Assim, $\alpha$ é um ponto isolado de $Y$.

\subsection{Um espaço de Ostaszewski}

Nesta seção vamos considerar o Princípio Diamante $\diamond$, introduzido na Seção 2.3.

Construiremos uma álgebra de Boole $A$ utilizando o Princípio $\diamond$ usando uma versão da álgebra $A^{\prime}$ construída na seção anterior. Por esse motivo, utilizaremos as notações da seção anterior. O objetivo da construção é obter um espaço compacto Hausdorff disperso $K$ tal que, $h d\left(K^{n}\right)=\omega$ para todo $n \in \omega$.

Pelo Lema 2.11, consideremos para cada $n \in \omega\left\{\Gamma_{\alpha}^{n}: \alpha<\omega_{1}\right\}$ tal que

- $\Gamma_{\alpha}^{n} \subset \underbrace{\alpha \times \cdots \times \alpha}_{n \text { vezes }}$,

- para todo $X \subset \omega_{1} \times \cdots \times \omega_{1}$,

$$
\left\{\alpha: X \cap \alpha \times \cdots \times \alpha=\Gamma_{\alpha}^{n}\right\},
$$

é estacionário. 
Vamos construir uma sequência $\left(A_{\alpha}, \mathfrak{u}_{\alpha}, x_{\alpha},(D(\alpha, n))_{n}\right)_{\alpha<\omega_{1}}$ tal que para cada $\alpha<\omega_{1}$ temos que $A_{\alpha}$ é uma subálgebra enumerável de $\wp(\mathbb{N})$ contendo FinCofin $(\mathbb{N}), \mathfrak{u}_{\alpha}$ é um ultrafiltro não principal de $A_{\alpha}, x_{\alpha}$ é um subconjunto de $\mathbb{N}$ e para todo $n \in \omega$ temos que $D(\alpha, n) \subset S(A)^{n}$ é tal que $\left(\mathfrak{u}_{\alpha}, \ldots, \mathfrak{u}_{\alpha}\right) \in \overline{D(\alpha, n)} S\left(A_{\alpha}\right)^{n}$.

- Seja $A_{0}=\operatorname{Fin} \operatorname{Cofin}(\mathbb{N})$ e consideremos em $A_{0}$ o ultrafiltro não principal $\mathfrak{u}_{0}=\operatorname{Cofin}(\mathbb{N})$.

Seja agora $\alpha<\omega_{1}$.

- Suponhamos que $\alpha=\beta+1$. Consideremos a ordem parcial $\mathbb{P}\left(A_{\beta}, \mathfrak{u}_{\beta}\right)$. Seja $n \in \omega$.

Se $\left(\mathfrak{u}_{\beta}, \ldots, \mathfrak{u}_{\beta}\right) \in \overline{\left\{\left(\delta_{1}^{* \beta}, \ldots, \delta_{n}^{* \beta}\right):\left(\delta_{1}, \ldots, \delta_{n}\right) \in \Gamma_{\beta}^{n}\right\}}\left(S\left(A_{\beta}\right)\right)^{n}$ e se cada elemento de $\Gamma_{\beta}^{n}$ tem todas as suas coordenadas distintas, definamos

$$
D(\beta, n)=\left\{\left(\delta_{1}^{* \beta}, \ldots, \delta_{n}^{* \beta}\right):\left(\delta_{1}, \ldots, \delta_{n}\right) \in \Gamma_{\beta}^{n}\right\} .
$$

Caso contrário, seja $D(\beta, n)=S\left(A_{\beta}\right)^{n} \backslash\left\{\left(\mathfrak{u}_{\beta}, \ldots, \mathfrak{u}_{\beta}\right)\right\}$.

Seja $G$ um filtro em $\mathbb{P}\left(A_{\beta}, \mathfrak{u}_{\beta}\right)$ que intersecta os densos da família:

$$
\begin{gathered}
\mathcal{E}^{1}\left(A_{\beta}, \mathfrak{u}_{\beta}\right) \cup \mathcal{E}^{2}\left(A_{\beta}, \mathfrak{u}_{\beta}\right) \cup \mathcal{E}^{3}\left(A_{\beta}, \mathfrak{u}_{\beta}\right) \cup \bigcup_{\delta \leq \beta} \mathcal{E}^{4}\left(A_{\beta}, \mathfrak{u}_{\beta}, D(\delta, 1)^{\prime}\right) \cup \\
\cup \bigcup_{\delta \leq \beta, n \in \omega} \mathcal{E}_{n}^{5}\left(A_{\beta}, \mathfrak{u}_{\beta}, D(\delta, n)^{\prime}\right) \cup \bigcup_{\delta \leq \beta, n \in \omega} \mathcal{E}_{n}^{6}\left(A_{\beta}, \mathfrak{u}_{\beta}, D(\delta, n)^{\prime}\right) .
\end{gathered}
$$

onde $D(\delta, n)^{\prime}=\left\{\left(\xi_{1}^{* \beta}, \ldots, \xi_{n}^{* \beta}\right):\left(\xi_{1}^{* \delta}, \ldots, \xi_{n}^{* \delta}\right) \in D(\delta, n)\right\}$ para todos $\delta \leq \beta$ e $n \in \omega$.

Definamos $x_{\beta}=x_{G}, A_{\beta+1}=\left\langle A_{\beta} \cup\left\{x_{\beta}\right\}\right\rangle$ e seja $\mathfrak{u}_{\beta+1}$ o ultrafiltro em $A_{\beta+1}$ gerado por $\mathfrak{u}_{\beta} \cup\left\{x_{\beta}\right\}$.

- Suponhamos que $\alpha$ é um ordinal limite. Definamos então $A_{\alpha}=\bigcup_{\lambda<\alpha} A_{\lambda}$ e $\mathfrak{u}_{\alpha}=\bigcup_{\lambda<\alpha} \mathfrak{u}_{\lambda}$.

Definamos por fim, $A=\bigcup_{\alpha<\omega_{1}} A_{\alpha}$ e seja $K=S(A)$.

Com exceção das famílias de subconjuntos densos obtidos em cada passo, a construção da álgebra $A$ é análoga a construção da álgebra $A^{\prime}$. Em particular, o Teorema 5.1 é verdadeiro para a álgebra $A$, ou seja, o compacto $K$ é disperso.

Vamos agora usar os densos definidos na construção para mostrar que $K$ e suas potências finitas são hereditariamente separáveis. Dado um subespaço $X$ de $K^{n}$, usaremos a propriedade reflexiva do Princípio Diamante para obter um subconjunto enumerável de $X$ que seja denso em $X$.

Os seguintes lemas serão utilizados no Teorema 5.3.

Lema 5.16. Seja $n \in \omega$. Se existe $\beta<\omega_{1}$ tal que cada elemento de $\Gamma_{\beta}^{n}$ possui todas as suas coordenadas distintas $e$

$$
\left(\mathfrak{u}_{\beta}, \ldots, \mathfrak{u}_{\beta}\right) \in \overline{\left\{\left(\delta_{1}^{* \beta}, \ldots, \delta_{n}^{* \beta}\right):\left(\delta_{1}, \ldots, \delta_{n}\right) \in \Gamma_{\beta}^{n}\right\}}\left(S\left(A_{\beta}\right)\right)^{n},
$$

então, para $\alpha_{1}, \ldots, \alpha_{n}>\beta$, temos que

$$
\left(\alpha_{1}, \ldots, \alpha_{n}\right) \in \overline{\left\{\left(\delta_{1}, \ldots, \delta_{n}\right):\left(\delta_{1}, \ldots, \delta_{n}\right) \in \Gamma_{\beta}^{n}\right\}}{ }^{S(A)^{n}} .
$$

Demonstração. Seja $\beta<\omega_{1}$ tal que

$$
\left(\mathfrak{u}_{\beta}, \ldots, \mathfrak{u}_{\beta}\right) \in \overline{\left\{\left(\delta_{1}^{* \beta}, \ldots, \delta_{n}^{* \beta}\right):\left(\delta_{1}, \ldots, \delta_{n}\right) \in \Gamma_{\beta}^{n}\right\}}\left(S\left(A_{\beta}\right)\right)^{n} .
$$

Pela construção da sequência $\left(A_{\alpha}, \mathfrak{u}_{\alpha}, x_{\alpha},(D(\alpha, n))_{n}\right)_{\alpha<\omega_{1}}$, segue que $D(\beta, n)=\left\{\left(\delta_{1}^{* \beta}, \ldots, \delta_{n}^{* \beta}\right)\right.$ : $\left.\left(\delta_{1}, \ldots, \delta_{n}\right) \in \Gamma_{\beta}^{n}\right\}$. Assim, para todo $\alpha \geq \beta$, temos que o filtro construído no passo $\alpha$ intersecta os 
densos das famílias

$$
\mathcal{E}^{4}\left(A_{\alpha}, \mathfrak{u}_{\alpha}, D(\beta, 1)^{\prime}\right), \mathcal{E}_{n}^{5}\left(A_{\alpha}, \mathfrak{u}_{\alpha}, D(\beta, n)^{\prime}\right), \mathcal{E}_{n}^{6}\left(A_{\alpha}, \mathfrak{u}_{\alpha}, D(\beta, n)^{\prime}\right) .
$$

Logo, pelos lemas $5.9,5.10,5.12,5.13$ temos que $\left(\alpha_{1}, \ldots, \alpha_{n}\right) \in \bar{\Gamma}_{\beta}^{n} S(A)^{n}$ para todos $\alpha_{1}, \ldots, \alpha_{n}>$ $\beta$.

Definição 5.10. Sejam $n \in \mathbb{N}$ e $\gamma$ um ordinal.

- Dados $t, s \in \gamma^{n}$, definimos $t \prec s$ se para todo $i=1, \ldots, n$, temos que $t(i)<s(i)$ e nesse caso dizemos que s dominat.

- Dado $X \subset \gamma^{n}$, dizemos que $X$ é dominante em $\gamma^{n}$ se para todo $t \in \gamma^{n}$ existe $s \in X$ tal que $t \prec s$.

Lema 5.17. Sejam $n \in \mathbb{N}$ e $X \subset K^{n}$ tal que $X$ é dominante em $\omega_{1}^{n}$. Então o conjunto

$$
C:=\left\{\beta:\left(\omega_{1}^{* \beta}, \ldots, \omega_{1}^{* \beta}\right) \in \overline{\left\{\left(\alpha_{1}^{* \beta}, \ldots, \alpha_{n}^{* \beta}\right):\left(\alpha_{1}, \ldots, \alpha_{n}\right) \in X \cap \beta^{n}\right\}}{ }^{S\left(A_{\beta}\right)^{n}}\right\}
$$

é club em $\omega_{1}$.

Demonstração. Vamos mostrar que $C$ é fechado e ilimitado.

Afirmação 1. $C$ é fechado.

Demonstração da Afirmação 1. Seja $\gamma$ limite e suponha que $C \cap \gamma$ é ilimitado em $\gamma$. Seja $s(a)$ uma vizinhança de $\omega_{1}^{* \gamma}$, i.e, $a \in \omega_{1}^{* \gamma} \subset A_{\gamma}=\bigcup_{\beta<\gamma} A_{\beta}$. Logo existe $\beta<\gamma$ tal que $a \in A_{\beta}$. Como $C \cap \gamma$ é ilimitado em $\gamma$, podemos supor $\beta \in C \cap \gamma$.

Agora $\omega_{1}^{* \gamma} \in s(a)$ em $S\left(A_{\gamma}\right)$, logo $\omega_{1}^{* \beta} \in s(a)$ em $S\left(A_{\beta}\right)$. Por hipótese, existe $\left(\alpha_{1}, \ldots, \alpha_{n}\right) \in$ $X \cap \beta^{n}$ tal que $\left(\alpha_{1}^{* \beta}, \ldots, \alpha_{n}^{* \beta}\right) \in s(a)^{n}$. Logo, $\left(\alpha_{1}^{* \gamma}, \ldots, \alpha_{n}^{* \gamma}\right) \in s(a)^{n}$ em $S\left(A_{\gamma}\right)$. Mostramos assim que

$$
s(a)^{n} \cap\left\{\left(\alpha_{1}^{* \gamma}, \ldots, \alpha_{n}^{* \gamma}\right):\left(\alpha_{1}, \ldots, \alpha_{n}\right) \in X \cap \gamma^{n}\right\} \neq \emptyset .
$$

Como $a \in \omega_{1}^{* \gamma}$ era arbitrário, segue que

$$
\left(\omega_{1}^{* \gamma}, \ldots, \omega_{1}^{* \gamma}\right) \in \overline{\left\{\left(\alpha_{1}^{* \gamma}, \ldots, \alpha_{n}^{* \gamma}\right):\left(\alpha_{1}, \ldots, \alpha_{n}\right) \in X \cap \gamma^{n}\right\}}{ }^{S\left(A_{\gamma}\right)^{n}} .
$$

Assim, $\gamma \in C$. Portanto $C$ é fechado, o que demonstra a afirmação.

Vamos mostrar agora que $C$ é ilimitado.

Afirmação 2. $C$ é ilimitado.

Demonstração da Afirmação 2. Definamos $F: \omega_{1} \rightarrow \omega_{1}$ por

$$
F(\beta):=\min \left\{\gamma<\omega_{1}: \forall t \in \beta^{n} \exists s \in X \cap \gamma^{n}(t \prec s)\right\} .
$$

Como $X$ é dominante em $\omega_{1}^{n}$ e $\beta^{n}$ é enumerável para todo $\beta<\omega_{1}$, temos que $F$ está bem definida. Seja

$$
C^{\prime}:=\{\alpha: F(\alpha) \subset \alpha\} .
$$

Vamos primeiramente mostrar que $C^{\prime} \subset C$. Consideremos $\gamma \in C^{\prime}$. Seja $s(a)^{n}$ uma vizinhança de $\left(\omega_{1}^{* \gamma}, \ldots, \omega_{1}^{* \gamma}\right)$. Como $\gamma$ é limite, existe $\beta<\gamma$ tal que $a \in \omega_{1}^{* \beta} \subset A_{\beta}$.

Assim $a \in \alpha^{* \gamma}$ para todo $\alpha>\beta$. Consideremos o elemento $t \in(\beta+1)^{n}$ dado por $t=(\beta, \ldots, \beta)$. Como $\gamma \in C^{\prime}$, segue-se que $F(\beta+1)<\alpha$. Portanto, existe $z \in X \cap F(\beta+1)^{n} \subset X \cap \gamma^{n}$ tal que $z$ domina $t$. Logo,

$$
\left(z(1)^{* \gamma}, \ldots, z(n)^{* \gamma}\right) \in\left\{\left(\alpha_{1}^{* \gamma}, \ldots, \alpha_{n}^{* \gamma}\right):\left(\alpha_{1}, \ldots, \alpha_{n}\right) \in X \cap \gamma^{n}\right\} \cap s(a)^{n} .
$$


Como $a \in \omega_{1}^{* \gamma}$ era arbitrário, concluímos que $\gamma \in C$. Portanto $C^{\prime} \subset C$. Agora $C^{\prime}$ é club, pois $C^{\prime}$ é um conjunto fechado formado por pontos que são fechados por $F$, logo é club ${ }^{1}$. Em particular é ilimitado. Concluímos portanto que $C$ é ilimitado.

Pelas Afirmações 1 e 2, concluímos que $C$ é club.

O nosso objetivo é demonstrar que $h d\left(K^{n}\right)=\omega$ para todo $n \in \omega$. Mostraremos primeiro que $h d(K)=\omega$ e a demonstração para $n>1$ sera feita por indução sobre os naturais.

Teorema 5.2. $h d(K)=\omega$.

Demonstração. Seja $X \subset K$ não enumerável. Vamos mostrar que $X$ é um subespaço separável. Observemos que, no caso $n=1$, basta que $X \subset K$ seja não enumerável para que $X$ seja dominante. Assim, pelo Lema 5.17, $C:=\left\{\beta: \omega_{1}^{* \beta} \in{\overline{\left\{\alpha^{* \beta}: \alpha \in X \cap \beta\right\}}}^{S\left(A_{\beta}\right)}\right\}$ é club.

Pelo Princípio Diamante, temos que $\left\{\beta: X \cap \beta=\Gamma_{\beta}^{1}\right\}$ é estacionário. Logo,

$$
\left\{\beta: \omega_{1}^{* \beta} \in{\overline{\left\{\alpha^{* \beta}: \alpha \in X \cap \beta\right\}}}^{S\left(A_{\beta}\right)}\right\} \cap\left\{\beta: X \cap \beta=\Gamma_{\beta}^{1}\right\} \neq \emptyset .
$$

Seja então $\beta<\omega_{1}$ tal que $\omega_{1}^{* \beta} \in{\overline{\left\{\alpha^{\beta}: \alpha \in \Gamma_{\beta}^{1}\right\}}}^{S\left(A_{\beta}\right)}$. Pelo Lema 5.16, temos que $\delta \in{\overline{\Gamma_{\beta}^{1}}}^{S(A)}$ para todo $\delta>\beta$. Segue que $D=\{\alpha: \alpha \in X \wedge \alpha \leq \beta\} \cup \Gamma_{\beta}^{1}$ é um denso enumerável em $X$.

Na demonstração, obtemos algo ainda mais forte, a saber:

Corolário 5.2. Dado $F \subset K$ fechado, temos que $F$ é enumerável ou existe $\alpha<\omega_{1}$ tal que $\left[\alpha, \omega_{1}\right] \subset F$.

Demonstração. Consequência da demonstração do teorema 5.2.

Estamos agora em posição de demonstrar que $K^{n}$ é hereditariamente separável para todo $n \in \omega$.

Teorema 5.3. $h d\left(K^{n}\right)=\omega$, para todo $n \in \omega$.

Demonstração. Vamos provar por indução em $n \in \omega$.

O caso $n=1$ é o teorema 5.2.

Suponhamos então o resultado válido para todo $j<n \operatorname{com} n \geq 2$. Seja $X \subset K^{n}$ não enumerável.

Afirmação 1. É suficiente supor que todos os elementos de $X$ têm coordenadas todas distintas.

Demonstração da Afirmação 1. Suponhamos o resultado válido para todo $X$ tal que cada elemento de $X$ tem coordenadas distintas. No caso geral, podemos escrever

$$
X=X^{\prime} \cup \bigcup_{1 \leq i<j \leq n} X_{i, j}
$$

onde $X^{\prime}$ possui elementos com coordenadas todas distintas e para todos os pares $i, j$ com $1 \leq i<$ $j \leq n$, temos que

$$
X_{i, j}:=\left\{x \in X: \pi_{i}(x)=\pi_{j}(x)\right\},
$$

onde $\pi_{i}: \omega_{1}^{n} \rightarrow \omega_{1}$ é a projeção na i-ésima coordenada.

Nesse caso, obtemos que $X^{\prime}$ é separável por hipótese, e para cada $1 \leq i<j \leq n$, temos que $X_{i, j}$ é homeomorfo a um subespaço de $K^{n-1}$.Pela hipótese de indução, segue-se que $X_{j}$ é separável. Logo, $X$ é separável.

Afirmação 2. É suficiente supor que $X$ é dominante em $\omega_{1}^{n}$.

\footnotetext{
${ }^{1}$ Ver [2], Proposição 8.3.4, página 158.
} 
Demonstração da Afirmação 2. De fato, se $X$ não é dominante em $\omega_{1}^{n}$, deve existir $f \in \omega_{1}^{n}$ tal que $f \nprec g$ para todo $g \in X$. Podemos portanto escrever

$$
X=\bigcup_{i} \bigcup_{\beta \in f(i)}\left(\pi_{i}^{-1}(\beta) \cap X\right) .
$$

Agora para cada $i=1, \ldots, n$ e $\beta<f(i)$ temos que $\pi_{i}^{-1}(\beta) \cap X$ é homeomorfo a $\left\{\left(x_{1}, \ldots, x_{i-1}, x_{i+1}, \ldots, x_{n}\right)\right.$ : $\left.\left(x_{1}, \ldots, x_{n}\right) \in \pi_{i}^{-1}(\beta) \cap X\right\} \subset K^{n-1}$, que por hipótese de indução é separável. Assim, $X$ é união enumerável de subespaços separáveis, logo é separável.

Pelas Afirmações 1 e 2, podemos supor que todos os elementos de $X$ têm coordenadas todas distintas e que $X$ é dominante em $\omega_{1}^{n}$. Pelo Lema 5.17 temos que

$$
C:=\left\{\beta:\left(\omega_{1}^{* \beta}, \ldots, \omega_{1}^{* \beta}\right) \in{\overline{\left\{\left(\alpha_{1}^{* \beta}, \ldots, \alpha_{n}^{* \beta}\right):\left(\alpha_{1}, \ldots, \alpha_{n}\right) \in X \cap \beta \times \cdots \times \beta\right\}}}^{S\left(A_{\beta}\right)^{n}}\right\}
$$

é club. Seja $\beta \in C \cap\left\{\alpha: X \cap \alpha \times \cdots \times \alpha=\Gamma_{\alpha}^{n}\right\}$. Então, temos que

$$
\left(\omega_{1}^{* \beta}, \ldots, \omega_{1}^{* \beta}\right) \in \overline{\left\{\left(\alpha_{1}^{* \beta}, \ldots, \alpha_{n}^{* \beta}\right):\left(\alpha_{1}, \ldots, \alpha_{n}\right) \in \Gamma_{\beta}^{n}\right\}}\left(S\left(A_{\beta}\right)\right)^{n} .
$$

Pelo Lema 5.16 segue-se que $\left(\delta_{1}, \ldots, \delta_{n}\right) \in{\overline{\Gamma_{\beta}^{n}}}^{S(A)^{n}}$ se $\delta_{i} \geq \beta$, para todo $i=1, \ldots, n$. Como $\Gamma_{\beta}^{n} \subset X$, podemos escrever $X=X_{1} \bigcup X_{2}$, onde $X_{1}={\overline{\Gamma_{\beta}^{n}}}^{X}=X \cap \overline{\Gamma_{\beta}^{n}} S(A)^{n}$ e $X_{2}=X \backslash X_{1}$. Logo $X_{1}$ é separável, pois $\Gamma_{\beta}^{n}$ é denso em $X_{1}$. Por outro lado temos que

$$
X_{2} \subset[\beta \times S(A) \times \cdots \times S(A)] \cup \cdots \cup[S(A) \times \cdots \times S(A) \times \beta] .
$$

e cada parcela acima é um espaço homeomorfo a $K^{n-1}$. Pela hipótese de indução segue-se que são hereditariamente separáveis. Concluímos assim que $X_{2}$ é separável. Por fim, como $X=X_{1} \bigcup X_{2}$, segue-se que $X$ é separável.

Pelos Teoremas 5.1 e 5.3, segue que $K$ é um compacto Hausdorff disperso e tal que $K^{n}$ é hereditariamente separável para todo $n \in \omega$.

Vamos agora definir a noção de espaço de Ostaszewski e verificar que $K$ possui um subespaço de Ostaszewski.

Definição 5.11. Um espaço Hausdorff $X$ é um espaço de Ostaszewski se, $X$ é um espaço não enumerável, regular, enumeravelmente compacto, não compacto e tal que todo fechado de $X$ é enumerável ou co-enumerável.

Observemos que o compacto $K$ satisfaz as propriedades da definição de espaço de Ostaszewski, exceto que é compacto. Vamos mostrar que $K$ possui um subespaço de Ostaszewski.

Definição 5.12. Definamos $K^{*}=K \backslash\left\{\omega_{1}\right\}$.

Proposição 5.1. $K^{*}$ não é um espaço de Lindelöf.

Demonstração. Suponhamos que $K^{*}$ seja um espaço de Lindelöf.

Temos que $K^{*}=\bigcup_{\alpha<\omega_{1}} s\left(\mathbb{N} \backslash x_{\alpha}\right)$. Assim, deve existir $N \subset \omega_{1}$ enumerável tal que

$$
K^{*}=\bigcup_{\alpha \in N} s\left(\mathbb{N} \backslash x_{\alpha}\right)
$$

Porém temos que

$$
\bigcup_{\alpha \in N} s\left(\mathbb{N} \backslash x_{\alpha}\right) \subset[0, \sup N]
$$

onde $\sup N<\omega_{1}$, o que é contradição pois $\sup N+1 \in K^{*}$. 
Proposição 5.2. O espaço $K^{*}$ é enumeravelmente compacto.

Demonstração. Seja $\left(B_{n}\right)_{n}$ uma cobertura enumerável de $K^{*}$. Pelo Corolário 5.2, dado $n \in \mathbb{N}$ temos que $B_{n}$ é enumerável ou existe $\alpha<\omega_{1}$ tal que $\left[\alpha, \omega_{1}\right) \subset B_{n}$. Como o espaço $K^{*}$ é não enumerável, deve existir $n_{0} \in \mathbb{N}$ e $\alpha<\omega_{1}$ tais que $\left[\alpha, \omega_{1}\right) \subset B_{n_{0}}$. Em particular, $K^{*} \backslash B_{n_{0}}$ é fechado em $K$ e $\left(B_{n} \cap s\left(\mathbb{N} \backslash x_{\alpha}\right)\right)_{n}$ é uma cobertura de $K^{*} \backslash B_{n_{0}}$.

Logo, deve existir $n_{1} \in \mathbb{N}$ tal que $K^{*} \backslash B_{n_{0}} \subset \bigcup_{i=1}^{n_{1}} B_{i}$. Concluímos portanto que

$$
K^{*}=\bigcup_{i=0}^{n_{1}} B_{i} .
$$

Corolário 5.3. Não existe uma sequência não trivial em $K$ convergindo para $\omega_{1}$.

Demonstração. De fato, suponhamos que exista uma sequência não trivial $\left(a_{n}\right)_{n}$ convergindo para $\omega_{1}$ em $K$. Então, a sequência $\left(a_{n}\right)_{n}$ não tem ponto de acumulação em $K^{*}$. Como $K^{*}$ é Hausdorff, devemos ter que $A=\left\{a_{n}: n \in \omega\right\}$ é um subespaço discreto e fechado. Pela Proposição 5.2, temos que $K^{*}$ é enumeravelmente compacto e portanto $A$ deve ser enumeravelmente compacto, pois é um subespaço fechado de $K^{*}$. Assim, $A$ é um subespaço enumeravelmente compacto de tamanho $\omega$ e discreto, o que é uma contradição.

Teorema 5.4. $K^{*}$ é um espaço de Ostaszewski.

Demonstração. Pela construção de $K$, temos que $K$ não é enumerável e, portanto, $K^{*}$ também não é enumerável. Como $K$ é regular e regularidade é uma propriedade hereditária para subespaços, temos que $K^{*}$ é um espaço regular. Pela Proposição 5.1, temos que $K^{*}$ não é um espaço compacto. Pela Proposição 5.2, $K^{*}$ é enumeravelmente compacto. Por fim, pelo Corolário 5.2, todo fechado de $K^{*}$ é enumerável ou co-enumerável.

\subsection{Propriedades do correspondente espaço de Banach $C(K)$}

Proposição 5.3. Seja $X$ um compacto disperso. Se $f$ é uma função contínua de $X$ em $\mathbb{R}$, então $f[X]$ é enumerável.

Demonstração. Corolário 12.26 de [7].

Teorema 5.5. Seja $f \in C(K)$. Então existem $\alpha<\omega_{1}$ e $r \in \mathbb{R}$ tais que $f\left(\left[\alpha, \omega_{1}\right]\right)=\{r\}$.

Demonstração. Seja $f \in C(K)$. Então $K=\bigcup_{r \in f[K]} f^{-1}(\{r\})$. Pela Proposição 5.3, temos que $f[K]$ é enumerável. Como $K$ é não enumerável, deve existir $r \in f[K]$ tal que $f^{-1}(\{r\})$ é não enumerável. Assim, $f^{-1}(\{r\})$ é fechado não enumerável. Pelo Corolário 5.2 existe $\alpha<\omega_{1}$ tal que $\left[\alpha, \omega_{1}\right] \subset f^{-1}(\{r\})$. Em particular, $f\left(\left[\alpha, \omega_{1}\right]\right)=\{r\}$.

Teorema 5.6. $h d\left(B_{C(K)^{*}}^{n}\right)=\omega$ para todo $n \in \omega$.

Demonstração. Pelo Teorema $5.3 h d\left(K^{n}\right)=\omega$, para todo $n \in \omega$. Assim pelo Lema 4.1 de [16] temos que $h d\left(B_{C(K)^{*}}^{n}\right)=\omega$ para todo $n \in \omega$.

Teorema 5.7. Seja $C(K)$ munido com a topologia fraca. Então $h L(C(K))=\omega$.

Demonstração. Segue-se dos Teoremas 7.3 e 7.4 de [21].

Teorema 5.8. $C(K)$ é um espaço de Banach não separável e biort $(C(K))=\omega$. 
Demonstração. Pela Proposição 17.10 de [14], temos que $|K|=\omega_{1}=w(K)$, logo $K$ não é metrizável e, portanto, $C(K)$ é um espaço de Banach não separável. Por outro lado, $K$ é um espaço disperso, tal que $K^{n}$ é hereditariamente separável para todo $n \in \omega$. Assim, pelo Teorema 3.10, temos que $C(K)$ não possui sistemas biortogonais não enumeráveis.

Assim, pelo Princípio Diamante, temos um exemplo de espaço de Banach $X$ não separável que não possui sistemas biortogonais não enumeráveis, ou seja, vale a desigualdade

$$
\operatorname{biort}(X)<d(X) \text {. }
$$

No caso de sistemas semibiortogonais, temos o seguinte teorema:

Teorema 5.9. $\operatorname{sbiort}(C(K))=\omega_{1}$.

Demonstração. Pelo Lema 5.15, para todo $\alpha<\omega_{1}$, temos que $\alpha \in s\left(\mathbb{N} \backslash x_{\alpha}\right) \subset \alpha+1$. Para cada $\alpha<\omega_{1}$, consideremos pelo Lema de Urysohn, uma função $f_{\alpha}: K \rightarrow[0,1]$ tal que $f_{\alpha}(\alpha)=1$ e $f_{\alpha}$ se anula fora de $s\left(\mathbb{N} \backslash x_{\alpha}\right)$. Temos assim que $\left(f_{\alpha}, \delta_{\alpha}\right)_{\alpha<\omega_{1}}$ é um sistema semibiortogonal em $C(K) \times C(K)^{*}$.

Observação 5.2. Segue de resultados em [18] que se $X$ é um espaço compacto disperso não metrizável, então $C(K)$ admite um sistema semibiortogonal não enumerável. 

UM ESPAÇO DE OSTASZEWSKI SOB O PRINCÍPIO DIAMANTE $\diamond$ 


\section{Referências Bibliográficas}

[1] J. Baumgartner and P. Komjath. Boolean algebras in which every chain and antichain is countable. Fund. Math, 111:125-133, 1981. 63

[2] K. Ciesielski. Set theory for the working mathematician. London Mathematical Society Student Texts, 1997. 22, 76

[3] G.W. Day. Free complete extensions of boolean algebras. Pacific. J. Math., 15:1145-1151, 1965. 7

[4] J. Diestel. Sequences and series in Banach spaces. Springer, graduate texts in mathematics, 92 edition, 1984. 4

[5] P. Enflo. A counterexample to the approximation property in Banach spaces. Acta Math, 130:309-317, 1973. xiii, 4, 25, 31

[6] R. Engelking. General Topology. Sigma Series in Pure Mathematics, 2nd edition, 1989. 3, 4

[7] M. Fabian, P. Habala, P. Hájel, V. Montesinos, and V. Zizler. Banach space theory - The basis for linear and nonlinear analysis. Springer, 2010. 1, 3, 5, 78

[8] M. Foreman, M. Magidor, and S. Shelah. Martin's maximum, satured ideals, and non-regular ultrafilters. Math. Ann., 127:1-47, 1988. 35

[9] F. Galvin. Chain conditions and products. Fund. Math, 108:33-48, 1980. 21

[10] P. Hájek, V. M. Santalucía, J. Vanderwerff, and V. Zizler. Biorthogonal systems in Banach spaces. Springer, 2000. 31

[11] R. Hodel. Cardinal functions. Handbook of set-theoretic topology, I:1-61, 1984. 26, 33, 50

[12] W. Johnson and H. Rosenthal. On $\mathrm{w}^{*}$ - basic sequences and their applications to the study of Banach spaces. Studia Math, 43:77-92, 1972. 5

[13] I. Juhász and Z. Szentmiklóssy. On d-separability of powers and $c_{p}(x)$. Topology Appl., 115:277-281, 2008. 61

[14] S. Koppelberg. Handbook of Boolean algebras. North-Holland Publishing Co, 1989. 6, 7, 59, 79

[15] P. Koszmider. On a problem of rolewicz about Banach spaces that admit support sets. J. Funct. Anal, 257:2723-2741, 2009. 39

[16] P. Koszmider. Some topological invariants and biorthogonal systems in Banach spaces. Extracta Math, 26(2):271-294, 2011. xiv, 50, 56, 78

[17] K. Kunen. Set theory. An introduction to independence proofs. North-Holland Publishing Co, 1980. $16,20,22$

[18] A.J. Lazar. Points of support for closed convex sets. Illinois Journal of Mathematics. 79 
[19] V.I. Malyhin. On the tightness and the souslin number of exp x and a product of spaces. Dokl. Akad. Nauk SSSR, 203:1001-1003, 1972. 33

[20] A.I. Markushevic. On a basis in the wide sense for linear spaces. Dokl. Akad. Nauk., 41:241244, 1943. 30

[21] S. Negrepontis. Banach spaces and topology. Handbook of Set-Theoretic Topology, NorthHolland, Amsterdam, pages 1045-1142, 1984. xiv, xv, 25, 38, 63, 78

[22] A.J. Ostaszewski. On countably compact, perfectly normal spaces. J. London Math. Soc, 14:505-516, 1976. xv, 63

[23] Z. Semadeni. Banach spaces of continuos functions. Monografie Matematyczne Tom 55 PWN Polish Scientific Publishers, Warsaw, 1971. 3

[24] S. Todorcevic. Irredundant sets in Boolean algebras. Trans. Amer. Math. Soc, 290:711-723, 1985. 49,51

[25] S. Todorcevic. Biorthogonal systems and quotient spaces via Baire category methods. Math. Ann., 335:687-715, 2006. xiv, 25, 31, 33, 35, 45

[26] P. Wojtaszczyk. Banach spaces for analysts. Cambridge Univsersity Press, 1991. 2 


\section{Índice Remissivo}

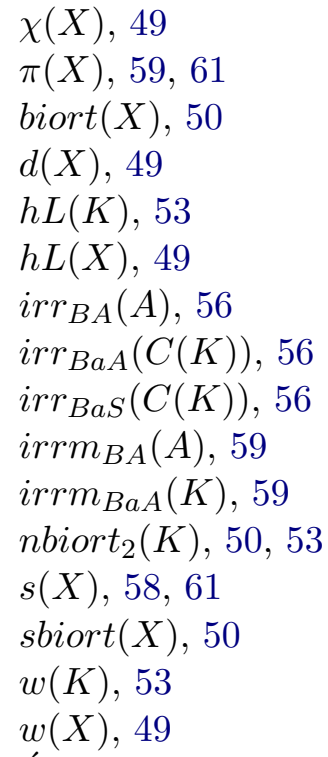

Densidade, 49

Denso, 15

Espaço disperso, 7, 73

Espaço hereditariamente Lindelöf, 36

Espaço hereditariamente separável, 36

Extensões simples, 7, 63

Filtro, 15

Grau de Lindelöf, 49

Hahn-Banach, 1

Irredundância, 56

Lema de Urysohn, 3
Ostaszewski, 77

Peso topológico, 49

Princípio Diamante, 22, 73

Sistema biortogonal, 26, 50

Sistema biortogonal nice, 50

Sistema semibiortogonal, 41

Teorema da Forma Normal, 6

Teorema de Alaoglu, 2

Teorema de Markushevic, 30

Teorema de McKenzie, 59

Teorema de Stone Weierstrass, 4

Topologia fraca, 1

Topologia fraca estrela, 2 